\title{
THE LONG-TERM IMPACTS OF HUNTING ON POPULATION VIABILITY OF WILD UNGULATES IN TARANGIRE, NORTHERN TANZANIA
}

\author{
Alfan Abeid Rija
}

A thesis submitted in partial fulfilment of the requirements for the degree of Master of Science in Conservation

Victoria University of Wellington

2009 


\begin{abstract}
Hunting through illegal and legal means is increasingly recognised as a threat to the sustainability of wildlife populations in reserves throughout Africa. Despite this, in Tanzania, legal hunting has persisted and serves as a source of revenue for conserving these species. Poaching remains a major threat to wildlife populations in many parks and reserves of Tanzania and wildlife habitats are diminishing due to human activities.
\end{abstract}

I examined the impacts of hunting on the long-term persistence of four wild ungulates; impala (Aepyceros melampus Matschie), Thomson's gazelle (Gazella thomsonii Günther), wildebeest (Connochaetes taurinus Thomas) and zebra (Equus burchellii Matschie) around Tarangire National Park, in northern Tanzania. I investigated the population sizes of these species using Distance sampling method and determined there were $4534 \pm 1393$ impala $1398 \pm 491$ Thomson's gazelle, $5199 \pm$ 2670 wildebeest, and $11223 \pm 4216$ zebra, in the study area. I obtained an estimate of the legal offtake over a five year period from data provided by hunting companies and districts offices in charge of hunting in the area to establish an average size of annual legal offtake of the area. I estimated the poaching levels for each species using random response method by interviewing 298 household respondents within communities living around the area. This established that illegal kill were 2-3 times higher than legal offtake for all four animal species except zebra. The total annual harvests were $6.6 \%$ for impala, $18.2 \%$ for Thomson's gazelle, $5.2 \%$ for wildebeest and $2 \%$ for zebra, of population sizes. Using literature review I obtained vital lifehistory parameters for each species either from within Tarangire, or elsewhere in East Africa.

The long-term viability of the four species was then examined under a computer program-VORTEX by constructing a series of models to test the effects of different hunting regimes. The models integrated mortality and fecundity rates, species population sizes and harvest (legal and illegal) rates. Of the four species, impala and Thomson's gazelle fared badly under all simulations, with up to $76 \%$ and $68 \%$ respectively of the modelled populations going extinct within 100 years under present 
hunting levels. Wildebeest and zebra were more resilient to hunting. Zebra populations remained robust under current hunting rates. However, its population will slowly decline towards extinction when the hunting exceeds the current rate of $2 \%$. The population of wildebeest will decline towards extinction if the current offtake of $5 \%$ persists. The impacts of illegal hunting are severe.

This study is the first attempt to characterise the dynamics of the harvested ungulate populations in Tarangire, Tanzania. Poor understanding of this ecosystem especially on the demographic variables of these species, limit firm conclusions. Nevertheless, the findings presented here suggest that VORTEX modelling may be a useful tool for managing hunting at Tarangire and for highlighting research priorities. 


\section{ACKNOWLEDGEMENTS}

Those who have contributed to bringing this thesis into print are too numerous to list them entirely.

First, I gratefully acknowledge support from New Zealand Aid for International Development Agency (NZAid) for providing me with a scholarship to pursue a MSc. degree for which this thesis is part of. Thanks to the Centre for Biodiversity and Restoration Ecology (CBRE) of Victoria University of Wellington for giving some funds to help me with field research in Tanzania. I also acknowledge a financial support from the School of Biological Sciences (SBS), Victoria University of Wellington to attend and present one of the thesis chapters at an international conference in Fremantle-Western Australia in 2008.

I wish to thank Tanzania National Parks Authority for allowing me to conduct research in Tarangire. To the Heads of villages; Sukuro, Lolkisale, Terrat and Kimotorok for generously permitting me to work with the village communities during my field work in Masailand.

My thesis supervisors have helped guide my research over the past two years, and were willing to forego what they were doing to fit me in during my thesis writing. Special thanks are due to Murray Williams who not only supervised my work but also, Murray was my mentor. At times things were so hard that I felt to quitting it but he was there and nagged me to keep at it. Murray Williams gave me unfailing support and pushed for me for financial support from SBS when needed. Wayne Linklater constantly took his precious time to provide me with valuable advice on improving this thesis. Thanks Murray for being a source of inspiration and true wisdom for me.

Many friends helped with the rigours of fieldwork without whose endurance, this work would have not been possible. Salum Ally is thanked for assisting me with the data collection. Salum endured the hassles of getting stuck in muddy roads is the Simanjiro plains while censuring wildlife and he communicated well with the Masai during interviews. Mabrouk Rajab and Haruna also rendered necessary assistance to me during data collection and were good companions at the field camp. L. Bushnot 
was particularly useful in communicating to fellow Masai during the interviews. I thank Eden Malisa for humbly accepting my request to hire his four-wheel drive vehicle for fieldwork in Tarangire. To the staff of Tarangire National Park for their generous welcome in Tarangire.

I thank the entire staff of SBS for providing me with the necessary assistance I needed when at the school. Many thanks to Victoria International staff panel for a warm welcome to New Zealand and for keeping me happy during my studies. Particular thanks to Bridget Nankivell, for working hard to get her job well done. Thanks to all colleagues in Kirk \# 702; Mark, Lisa, Kuruthumu, Heather, Fiona and Chris for a laugher especially at times when things were a bit more overwhelming.

Thanks to my family for encouragement and patience while away pursuing this degree for which they are proud of.

This thesis is dedicated to all people, whose breasts are deeply implanted with spirits of hunting! 


\section{TABLE OF CONTENTS}

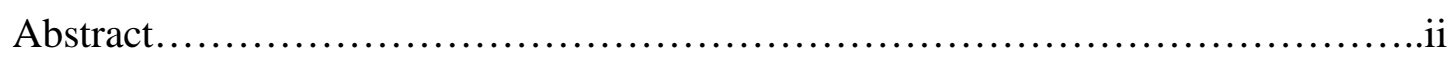

Acknowledgement........................................................ iv

The long-term impacts of hunting on population viability of wild

ungulates in Tarangire, Northern Tanzania: Background and

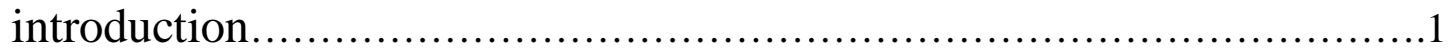

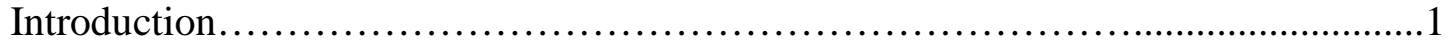

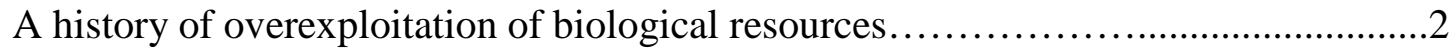

Wildlife management system in Tanzania..................................................

Consumptive use of wildlife in Tanzania......................................... 3

Framing the issue ................................................................

Research goal and objectives................................................6

Study site..........................................................

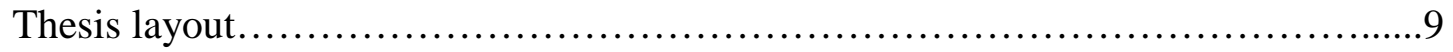

Chapter summary.............................................................

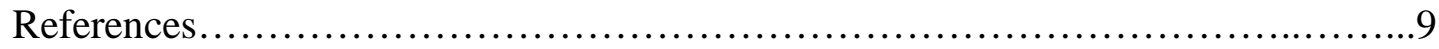

Legal hunting of wildlife outside of Tarangire National Park,

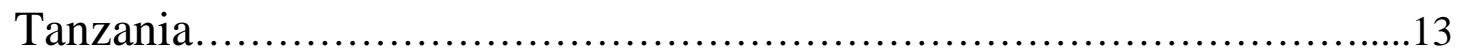

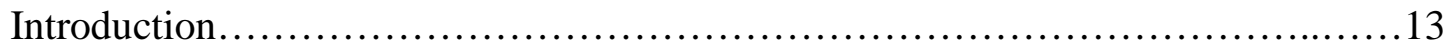

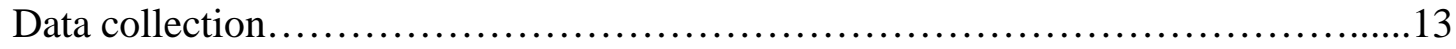

Results................................................................. 14

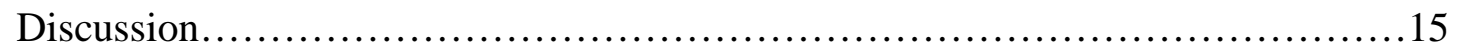

Chapter summary.......................................................... 17

References............................................................ 17

Poaching outside Tarangire national park, Tanzania: estimating illegal hunting of wildlife using the random response technique..............18

Abstract................................................................. 18

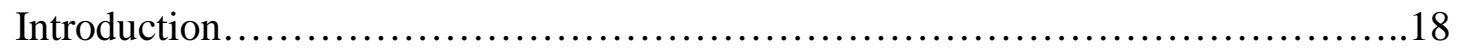

Use of Randomised Response Technique in surveys of sensitive issues.............20 
Application of RRT in the field of natural resources conservation.

Methods .23

Study site .23

The communities...................................................23

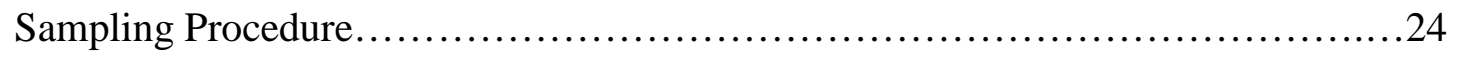

Administering the Randomised Response Technique and the Questionnaire

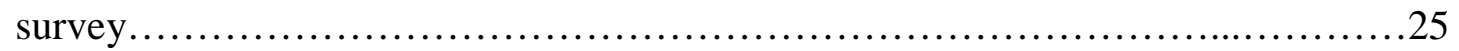

Data Analysis..........................................................28

Results.................................................................... 30

Poaching estimates of the wild ungulates....................................30

Respondent's demographics.......................................... 31

The magnitude of wildlife threats to the local people........................... 31

The benefits of wildlife resources to the local human population...................32

Local peoples' opinions on the trend of wildlife population and hunting in the

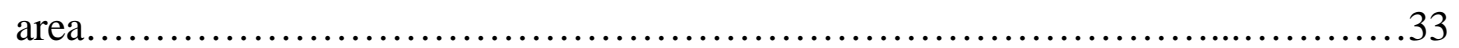

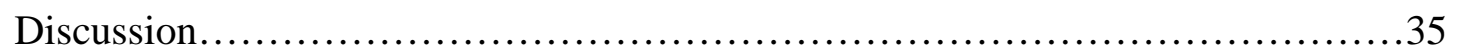

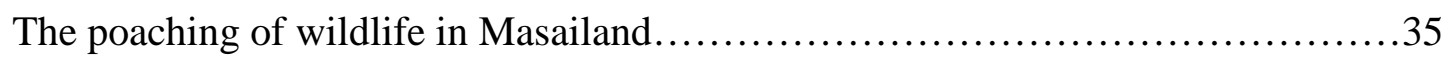

Wildlife and the local people in Masailand...................................36

The effect of the double-barrelled question on the precision of poaching estimates................................................................ 37

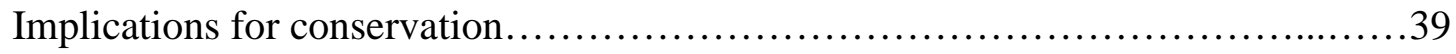

Appendix 3.0 Case study animal species used in this thesis......................40

Appendix 3.1 Questionnaire survey used along with the Random Response technique (RRT) in the study of poaching of wildlife outside Tarangire-northern Tanzania-

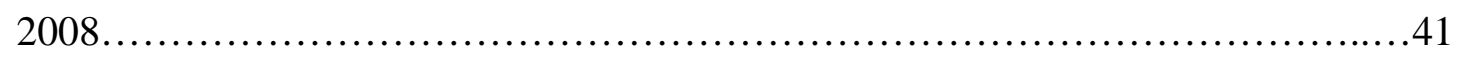

References............................................................. 43

Life history parameters of zebra, impala, Thomson's gazelle and wildebeest in the Tarangire area,

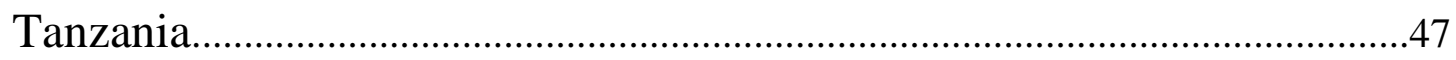

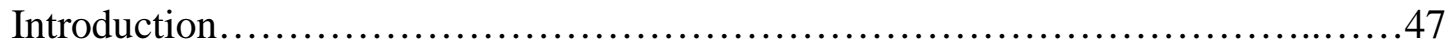

Life-history details of plains zebra........................................47

Life-history details of impala..............................................50

Life-history details of Thomson's gazelle.................................... 51 
Life-history details of wildebeest..........................................52

Size and life-history (Allometric relationships) ..............................54

Chapter summary..................................................... 55

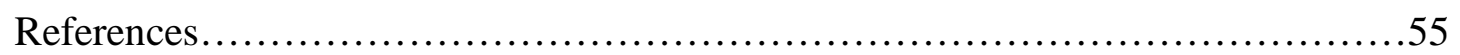

Density of wildlife populations outside Tarangire National Park,

Tanzania.............................................................. 58

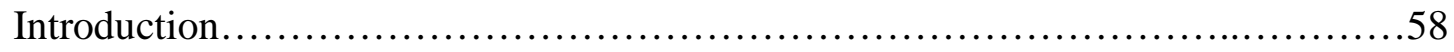

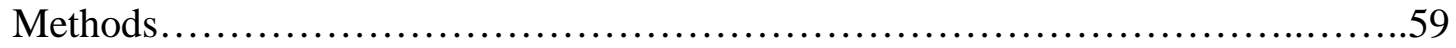

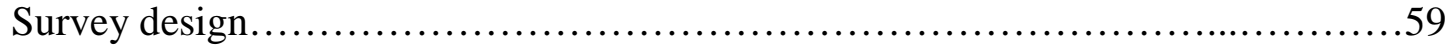

Data processing and analysis..................................................

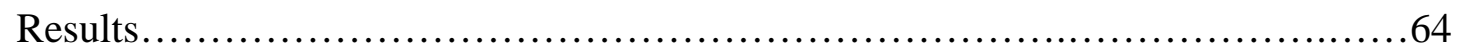

Density of animals in the area............................................64

Mean cluster size of the animals..........................................64

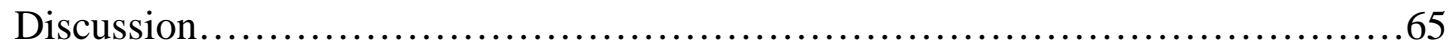

Zebra population.........................................................65

Wildebeest Population....................................................67

Impala and Thomson's gazelle populations..................................67

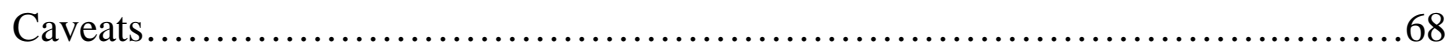

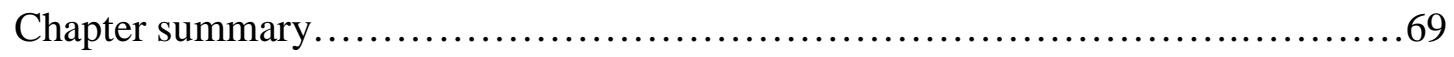

References............................................................. 69

Modelling the impact of hunting on species population viability.........71

Introduction........................................................ 71

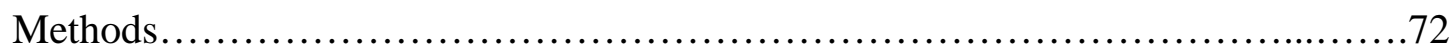

Sources of data......................................................... 72

Model Construction...................................................... 74

Zebra PVA................................................................. 74

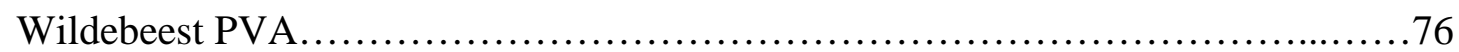

Thomson's gazelle PVA.................................................... 76

Impala PVA......................................................... 77

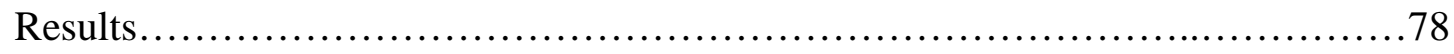


Dynamics of animal populations under no harvest.................................. 78

Effects of hunting on population viability.................................79

Effects of varying mortality and fecundity rates on population dynamics............82

Sensitivity test.................................................... 82

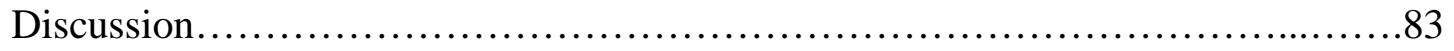

Model Caveats........................................................... 85

Implications for conservation and sustainable harvest in Tarangire ................87

Practical application of VORTEX for managing Tarangire wildlife populations.......88

Chapter summary ........................................................90

References....................................................................

\section{LIST OF TABLES AND FIGURES}

\section{Tables}

Table 2.0: Wildlife killed by citizen and tourist hunters over the five-year period 2003-2007 in the study site.

Table 3.0: Estimated poaching levels of four wildlife species in Masailand,

Tanzania.

Table 3.1: Estimated numbers of animals killed from the study area by illegal hunters on average in one year in comparison to legal offtake....

Table 3.2: The percentage of wildlife threats reported by the local people and the number of respondents (in brackets)....

Table 3.3 Respondents' yes (no) answers $(\mathrm{N}=298)$ on the question of whether or not they get direct benefit from the wildlife, whether or not were employed in the wildlife business, or get allowance as wildlife benefits from the government or hunting companies around the area.

Table 3.4a: Respondents' opinions in percentage, \% (n) on why people would tend to kill the wildlife from around the area. .34

Table 3.4b: Respondents' answers in \% (n) for the question what should the wildlife law do to protect the wildlife around the area....

Table 4.0: Demographic variables from published literature for plain zebra populations throughout the eastern and southern African range. 
Table 4.1: Survival and fecundity rates of zebra population in Serengeti National Park.

Table 4.2: Life history parameters of the eastern impala population.

Table 4.3: Life-history parameters of Thomson's gazelle.

Table 4.4: Life-history parameters of wildebeest from Eastern Africa. .53

Table 4.5: Life history parameters of target animals from around the species range.

Table 5.0: Model performance and selection statistics for the zebra, impala,

Thomson's gazelle and wildebeest distance data showing density

estimates

Table 5.1: Density and abundance of wildlife species in the study area....

Table 5.2: Estimated mean herd size, encounter rate (herds $\left./ \mathrm{km}^{2}\right)$ and density of cluster with standard errors based on the final analysis after truncation

Table 5.3: Population estimates of wildlife in the Simanjiro plains from previous studies conducted using aerial census techniques in comparison with the present study....

Table 6.0: Mean population sizes ( \pm standard errors) and species harvests for the Tarangire hunting area which were used in the models

Table 6.1: The ranges for the life history parameters of zebra as recorded in literature, and the values used in models in this study.

Table 6.2: The ranges for the life history parameters of wildebeest as recorded in literature, and the values used in models in this study.... 76

Table 6.3: The ranges for the life history parameters of Thomson's gazelle as recorded in literature and the values used in models in this study. ...

Table 6.4: The ranges for the life history parameters of impala as recorded in literature and the values used in models in this study. .78

Table 6.5a: Vortex simulation models for zebra population under different hunting scenarios showing the population growth $(\mathrm{r} \pm \mathrm{SD})$, probability of extinction, mean extinction time and mean final population $( \pm \mathrm{SD})$. .79 
Table 6.5b: Vortex simulation models for wildebeest population under different hunting scenarios showing the population growth $(\mathrm{r} \pm \mathrm{SD})$, probability of extinction, mean extinction time and mean final population $( \pm \mathrm{SD})$.

Table 6.5c: Vortex simulation models for Thomson's gazelle population under different hunting scenarios showing the population growth $(r \pm S D)$, probability of extinction, mean extinction time and mean final population $( \pm \mathrm{SD})$.

Table 6.5d: Vortex simulation models for the impala population under different hunting scenarios showing the population growth $(\mathrm{r} \pm \mathrm{SD})$, probability of extinction, mean extinction time and mean final population $( \pm \mathrm{SD})$

Table 6.6: Sensitivity of survival rates to increases of mortality (calf and yearling) and variation of mortality and fecundity rates.

\section{Figures}

Figure 1: Study area showing hunting areas outside Tarangire National Park,

Tanzania

Figure 2: Location of Simanjiro study site showing distribution of transects

(beaded lines) in the area.

Figure 3: Observed frequency histogram of truncated distance data for zebra, impala,

Thomson's gazelle and

wildebeest. 


\section{NORTHERN TANZANIA: BACKGROUND AND INTRODUCTION}

\section{Introduction}

Wildlife provides economic and social security as well as meeting the food and livelihood requirements of human communities in many biodiversity- rich areas of the world (Barnett, 2000; Bennett et al., 2002). Wild meat is a significant source of easily accessible animal protein among impoverished rural communities throughout Africa, Asia and Latin America (Rao \& McGowan, 2002). An increasing trade in wildlife bush meat (Davies, 2002; Rao \& McGowan, 2002) suggests that it is an important ingredient in food recipes of urban communities.

There is a high economic and sport value attached to the subsistence use of and commercial trade in wildlife resources, contributing significantly to the local and national economies. For example, it is estimated that the trophy hunting industry generates gross revenues of at least US\$ 201 million and US\$33-39 million per year respectively, in sub-Saharan Africa and Eurasia (Hofer, 2002; Lindsey et al., 2007). The growing ecotourism industry in many wildlife reserves and privately owned lands in Africa has proven to create income for these areas (Lindsey et al., 2007). Moreover, although it is largely ignored in national accounting processes, the combined economic value of wildlife from legal and illegal uses contribute significantly to the local economies of rural and urban human communities. For instance the rural communities of most west and central, and eastern Africa derive their annual income largely from trading wild meat (Kaltenborn et al., 2005; Noss, 2002; Robinson \& Bennett, 2004). In Liberia, 75\% of the country's meat comes from wild animals [Anstey in (Rao \& McGowan, 2002)].

As human populations continue to grow, pressures on wild species and natural ecosystems are becoming increasingly severe. With the increasing global demands for food security (Balmford \& Bond, 2005) and given the importance of wild animals to the human population, it is increasingly becoming difficult to manage wild nature without the consent of resident people. In the past two decades we have seen a paradigm shift in resource conservation systems from more exclusionary protectionist 
policies where strict rules, fine and fences were characteristic to a system that allows involvement of local people. Currently there is an increasing realisation that conservation by the people and for the people with careful regulated sustainable use of the resource will ensure long term perseverance of wildlife and habitats especially in countries with weak economies (Hackel, 1999; Songorwa et al., 2000; Wells \& Brandon, 1992). Many African countries, including Tanzania, have adopted this system and there has been substantial development largely accompanied by regulated use of wildlife resources.

\section{A history of overexploitation of biological resources}

Any use of biological resources will impact on the resource, whether negatively or positively. The use of modern technologies in hunting practices, and the increasing commercialisation of hunting are critical factors driving overexploitation and unsustainable use of wildlife in many ecosystems (Robinson \& Bennett, 2000). Several documented cases of overfishing leading to extinctions and collapse of coastal ecosystems provide lessons of misguided management of a natural resource that had sustained large human populations for long times (Jackson et al., 2001; Ludwig et al., 1993). Overexploitation has resulted in the ecological collapse and extinction of large animals and birds of North and South America (Olson \& James, 1982; Redford, 1992). In 1980, hunting contributed to drastic reductions in population of dorcas gazelle (Gazella dorcas) and to extermination of the Nubian bustard (Neotis nuba) from Sahelian Africa (Newby, 1990). More recently, hunting by humans has exterminated Miss Waldron's red colobus monkey (Procolobus badius waldroni) from West Africa forest (Oates et al., 2000).

On the other hand, there are places where hunting has been regulated successfully. The wild turkey (Meleagris gallopave); [Dickson, 1992 in (Loveridge et al., 2006)] white- tailed deer (Odocoileus virginianus) (Woolf \& Roseberry, 1998) and beaver (Castor Canadensis) [Novak 1987 cited in Loveridge et al. (2006) ] in North America are all species whose fortunes have been dramatically improved by a programme of conservation measures that include regulated harvests.

The management of wildlife in many African countries (including Tanzania) is constrained by meagre funds for running conservation activities. As a consequence, 
commercial hunting is always considered both as a conservation tool as well as for generating national incomes. Under such circumstance, market forces and internal pressures (e.g. poverty) could substantially influence the conservation and management of these resources negatively.

\section{Wildlife management system in Tanzania}

Wildlife is managed primarily through a system of national parks, game reserves and other areas owned by the government, and a range of wildlife management areas largely owned by the local community authorities. National parks (NP) are areas regarded of high conservation status and are mostly managed through protection. There is no direct use of resources by humans other than ecotourism (visual enjoyment, photographing and filming). Game reserves (GR) allow regulated hunting of wildlife from them with the prohibition of other human activities, such as crop cultivation and housing except for game reserve staff houses. The major uses of game reserves are trophy hunting, photographic and filming activities. Associated with these are Game controlled areas (GCA), where the hunting of wildlife, photographic and filming and human activities (livestock grazing and farming) is permissible. Open areas $(\mathrm{OA})$ ranks fourth and least in the group. OA have no formal conservation status and allow all human activities as in the third category. Such areas may be leased to hunting companies (URT, 1998).

There are different authorities overseeing the conservation of wildlife in these protected areas categories. All the national parks are managed by the Tanzania National Parks Authority (TANAPA). The game reserves and game controlled areas are controlled by the Wildlife Division (WD), (a division within the Ministry of Natural Resources and Tourism). Open areas and other designated wildlife management areas are managed by the respective district local authorities. Despite these categories and management authorities, the wildlife belongs to the state regardless of where it occurs (URT, 1974).

\section{Consumptive use of wildlife in Tanzania}

The commercial consumptive use of wildlife in Tanzania has persisted for over three decades, having started in the mid 1960s. Since then there has been a substantial growth in the industry accompanied by increasing hunting areas from just one in 1965 
to more than 130 presently (Baldus \& Cauldwell, 2004). This development had come about because of the increasing demands to obtain benefits from the wildlife and the need for funds to manage the wildlife.

Trophy hunting takes place on all protected area categories except in national parks. The legal hunting business occurs between $1^{\text {st }}$ July and $31^{\text {st }}$ December each year. Trophy hunting is controlled and coordinated by the WD. It allocates the hunting areas (blocks or concessions) to foreign or resident companies which bring in trophy hunters. There may be three or more hunting blocks in a single reserve depending on the reserve size and the population of the wildlife. The WD decides on the size of the quotas and the species to be hunted from individual blocks leased to hunting companies as well as from other hunting areas under districts level (see Baldus \& Cauldwell, 2004 for details). Based on the size of quotas decided, the WD issues the hunting permits to the clients and supervises the hunting process through to completion. Only adult male animals may be hunted in Tanzania.

Hunting permits for citizens (local subsistence hunting) are issued by the respective regional or district game officers in which hunting is carried out. In this case all the game species hunted must conform to the quotas allocated to them by the WD. When the hunting season closes, the districts game offices report back to WD describing how the quotas were utilised and what funds were generated from the selling of the wildlife. The information about all the hunted animals from different reserves is kept by the WD and a replicate copy retained in respective district game offices managing these reserves. Subsistence hunting information can be accessed directly from the district game offices too.

\section{Framing the issue}

Whilst the hunting industry in Tanzania is growing steadily, its operational aspects are worth looking at;

- Setting hunting quotas. Determining quotas for an area is largely a process of educated guess work (Caro et al., 1998b). The Wildlife Division decides on quotas using data from aerial census and past hunting records, ideas from professional hunters and outfitters, and suggestions from reserve managers and district wildlife officers of respective hunting areas (Baldus \& Cauldwell, 
2004). Ideally, all these sources of data should be integrated to confirm the population status of the wildlife and result in good decision making. The downside of it however, is that there may be less scrupulous people (among those giving suggestions) who just want to make money out of wildlife. This can lead to large quotas being suggested for some areas that should otherwise not be. Furthermore, the shortage of funds for running conservation activities (e.g. patrolling reserves) at district and local levels could encourage them to suggest bigger quotas. Thus monetary gain from selling more wildlife would be a priority especially when a large proportion of the funds are retained for district official activities.

- Increased concession (blocks) areas. There has been an increase in the number of hunting blocks, some of which are newly formed, while others originate from subdividing the pre-existing ones (Baldus \& Cauldwell, 2004). While this is happening there have been no reductions in the quotas for some of the subdivided hunting blocks (Baldus \& Cauldwell, 2004). This means that the quota size has remained the same on each of the sub-blocks as the original "mother block", consequently increasing the number of animals hunted overall.

- There is little monitoring of the population trend of the wildlife by those who decide a quota size. Monitoring of the wildlife population is an expensive undertaking and most wildlife officers do not have the skills required. The WD conducts some regular aerial population surveys around major national parks but does not cover all the hunting areas (Caro et al., 1998b). There are little data available for all the hunting areas on which to make good decisions.

- Quota setting processes rarely take into account the animals killed illegally. There is an extensive body of literature on the poaching of wildlife in Tanzania, e.g. (Hofer et al., 1996; Holmern et al., 2007; Loibooki et al., 2002), and its effects on the population of wildlife is immense. However, the rate at which poaching removes wildlife is not known and often is ignored in the management process.

- Most local (citizen) hunting is unsupervised by district game officers. As for the trophy hunting, besides being supervised, there are circumstances when supervisors are less careful and would not stop any misconduct by the hunters. 
The implications of this are that there may be overshooting of the animals allocated on the permits by the hunters as well as hunting species not allocated or paid for. For example using sex-specific molecular makers to examine the gender of the hunted leopards from Tanzania, it was found that $28.6 \%$ were females although all the skin trophies were tagged males (Spong et al., 2000).

These actual or potential irregularities are a cause for concern if the hunting industry is to be sustainable. Recent studies (Caro et al., 1998b; Stoner et al., 2007) point out that the population of wildlife has declined over many reserves and attribute hunting as the ultimate cause of the declines. While these studies provide insights into understanding the effects of hunting they do not account for the effect of the wild animals taken illegally nor do they account for the natural deaths of animals in the reserves. Furthermore, they do not show the overall long-term impacts of hunting on the species populations.

\section{Research goal and objectives}

The goal of this study was to examine whether or not current hunting rates are sustainable by developing a population viability analysis (PVA). To fulfil this goal, I addressed the following objectives.

1. To determine the current population density and abundance of four principal game species in the Tarangire hunting area

2. From existing literature, determine the vital demographic parameters of the four principal game species.

3. To determine the size of current legal harvest of the four principal game species.

4. To investigate the current rate of illegal harvest of the four principal game animals.

5. To assess the population viability of the four principal game species under current hunting (legal and illegal) levels.

I have chosen to study the Tarangire hunting area in northern Tanzania as a case example of what may be happening elsewhere in Tanzania and to examine hunting impact on the four most commonly hunted game species: zebra (Equus burchellii Matschie), impala (Aepyceros melampus Matschie), wildebeest (Connochaetes 
taurinus Thomas) and Thomson's gazelle (Gazella thomsonii Günther). The approach taken in this study may be applicable across other areas facing similar situations in Tanzania, and elsewhere in Africa.

\section{Study site}

This study was conducted in areas outside of Tarangire National Park, northern Tanzania (Fig. 1). The study site comprises a game controlled area (Lolkisale), an open area to the east, and a game reserve (Mkungunero) to the south of Tarangire National Park. It is surrounded by human habitations and sanctions human activities such as agriculture and livestock keeping. With an area of $570 \mathrm{~km}^{2}$, it harbours numerous wildlife species and has been subdivided into hunting concessions and leased to hunting companies by the WD. There is hunting of wildlife done from this area by both legal hunters (foreign and residents) as well as illegal hunters (poachers).

The area is semi-arid and receives annual rainfalls ranging between $450-600 \mathrm{~mm}$. The vegetation structure is mostly short grass plains mixed with woodland, shrubs and thorny bushes, characteristic of savannah (Kahurananga, 1979). The unreliable and erratic precipitation in the area renders it support vegetations suitable for livestock grazing.

The inhabitants are mostly the Masai people who are livestock keepers. Because of occupying this range, it is commonly referred to as "Masailand" (land of Masai people). In this study, this term was used also when referring to the hunting areas outside of Tarangire National Park. 


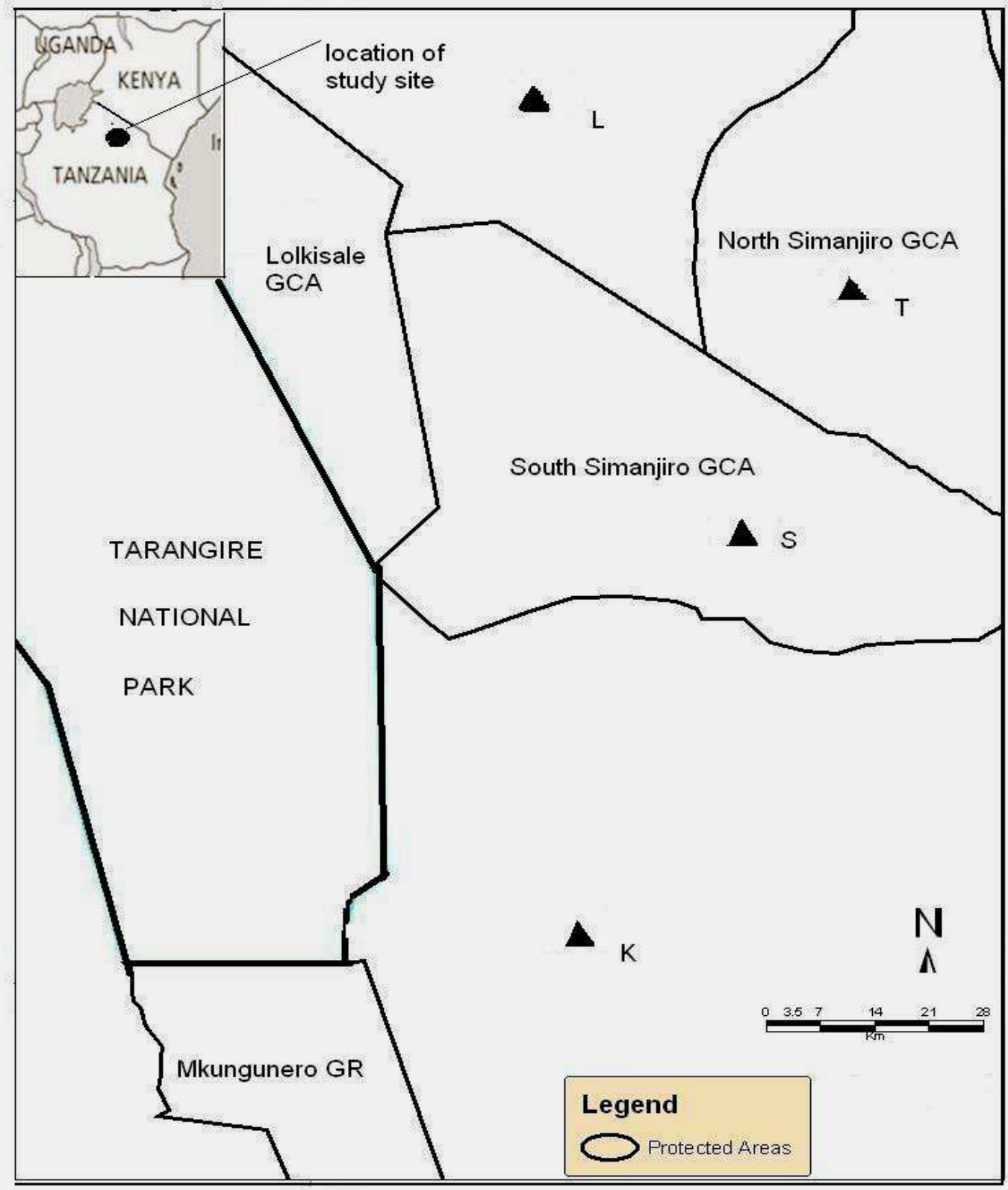

Figure 1: Study site showing hunting areas (game reserves, GR, and game control areas, GCR) outside the Tarangire National Park, Tanzania and the villages (triangles), of Lolkisale (L), Terrat (T), Sukuro (S), and Kimotorok $(K)$ located within the area. 


\section{Thesis layout}

The outcomes for each of the research objectives have been presented as stand-alone chapters in anticipation of possible subsequent publication of the chapters as papers. Each chapter therefore has its own discussion section, summary and reference list. Chapter 3 has a more formal presentation ahead of its submission for publication. The last chapter summarises main findings and suggests management options to achieve sustainability of the wildlife in the Tarangire region.

\section{Chapter summary}

This chapter introduces salient issues surrounding the hunting of wild game animals in Tanzania in general and Tarangire National Park in particular. Quota setting in the absence of reliable information on population size and recent trend is highlighted as a significant problem, as too is the extent of illegal hunting. I pose research questions which may assist better management of hunting and I briefly describe approaches to answering each question that will be expanded upon within this thesis.

\section{References}

Baldus, R.D., \& Cauldwell, A.E. (2004). Tourist hunting and its role in development of wildlife management areas in Tanzania. $\underline{\text { www.cic- }}$ wildlife.org/uploads/media/Hunting_Tourism.pdf (accessed on 29, July 2008).

Balmford, A., \& Bond, W. (2005). Trends in the state of nature and their implications for human well-being. Ecology Letters, 8(11), 1218-1234.

Barnett, R. (2000). Wildlife meat utilisation in the east and southern Africa region. In: links between biodiversity conservation, livelihoods and food security. The sustainable use of wild species for meat.(Eds S. Mainka and M.Trivedi. The IUCN Species survival commission , TRAFFIC East/Southern Africa, Nairobi, Kenya. .

Bennett, E.,Eves, H.,Robinson, J., \& Wilkie, D. (2002). Why is eating bushmeat a biodiversity crisis? Conservation in Practice, 3, 28-29.

Caro, T.M.,Pelkey, N.,Borner, M.,Severre, E.L.M.,Campbell, K.L.I., et al. (1998b). The impact of tourist hunting on large mammals in Tanzania: an initial assessment. African Journal of Ecology, 36(4), 321-346. 
Davies, G. (2002). Bushmeat and international development. Conservation Biology, 16(3), 587-589.

Hackel, J.D. (1999). Community conservation and the future of Africa's wildlife. Conservation Biology, 13(4), 726-734.

Hofer, D. (2002). The lions share of the hunt.Trophy hunting and conservation: a review of the legal Eurasian tourist hunting market and trophy trade under CITES.TRAFFIC Europe report, 72pp.

Hofer, H.,Campbell, K.L.I.,East, M., \& Huish, S.A. (1996). The impact of game meat hunting on target and non-target species in the Serengeti. In The exploitation of mammal populations: 117-146. Taylor, V. J. \& Dunstone, N. (Eds).London: Chapman and Hall.

Holmern, T.,Muya, J., \& Røskaft, E. (2007). Local law enforcement and illegal bushmeat hunting outside the Serengeti National Park, Tanzania. Environmental Conservation, 34(01), 55-63.

Jackson, J.B.C.,Kirby, M.X.,Berger, W.H.,Bjorndal, K.A.,Botsford, L.W., et al. (2001). Historical overfishing and the recent collapse of coastal ecosystems. Science, 293, 629-638.

Kahurananga, J. (1979). The vegetation of the Simanjiro plains, northern Tanzania. African Journal of Ecology, 17(2), 65-83.

Kaltenborn, B.,Nyahongo, J., \& Tingstad, K. (2005). The nature of hunting around the western corridor of Serengeti national park, Tanzania. European Journal of Wildlife Research, 51(4), 213-222.

Lindsey, P.A.,Roulet, P.A., \& Romañach, S.S. (2007). Economic and conservation significance of the trophy hunting industry in sub-Saharan Africa. Biological Conservation, 134(4), 455-469.

Loibooki, M.,Hofer, H.,Campbell, K.L.I., \& East, M.L. (2002). Bushmeat hunting by communities adjacent to the Serengeti national park, Tanzania: the importance of livestock ownership and alternative sources of protein and income. Environmental Conservation, 29(3), 391-398.

Loveridge, A.J.,Reynolds, J.C., \& Milner-Gulland, E.J. (2006). Is sport hunting part of conservation? Pp. 224-240 In: D.W. Macdonald and K. Service (Eds), Key Topics in Conservation, Blackwell, Oxford..

Ludwig, D.,Hilborn, R., \& Waters, C. (1993). Uncertainty, resource exploitation, and conservation: lessons from history. Science, 260, 17-36. 
Newby, J.E. (1990). The slaughter of Sahelian wildlife by Arab royalty. Oryx, 24(1), 6-8.

Noss, A.J. (2002). Cable snares and bushmeat markets in a central African forest. Environmental Conservation, 25(3), 228-233.

Oates, J.F.,Abedi-Lartey, M.,McGraw, W.S.,Struhsaker, T.T., \& Whitesides, G.H. (2000). Extinction of a West African red colobus monkey. Conservation Biology, 14, 1526-1532.

Olson, S.L., \& James, H.F. (1982). Fossil birds from the Hawaiian islands: evidence for wholesale extinction by man before western contact. Science, 217, 633635.

Rao, M., \& McGowan, P.J.K. (2002). Wild-meat use, food security, livelihoods, and conservation. Conservation Biology, 16(3), 580-583.

Redford, K.H. (1992). The empty forest. BioScience, 42, 412-422.

Robinson, J.G., \& Bennett, E.L. (2000). Hunting for sustainability in tropical forests. Columbia University Press, New York.

Robinson, J.G., \& Bennett, E.L. (2004). Having your wildlife and eating it too: an analysis of hunting sustainability across tropical ecosystems. Animal Conservation, 7(4), 397-408.

Songorwa, A.N.,Buhrs, T., \& Hughey, K.F.D. (2000). Community-based wildlife management in Africa: a critical assessment of the literature. . Natural Resourse Journal 40(3), 603-643.

Spong, G.,Hellborg, L., \& Creel, S. (2000). Sex ratio of leopards taken in trophy hunting: genetic data from Tanzania. Conservation Genetics, 1(2), 169-171.

Stoner, C.,Caro, T.,Mduma, S.,Mlingwa, C.,Sabuni, G., et al. (2007). Changes in large herbivore populations across large areas of Tanzania. African Journal of Ecology, 45(2), 202-215.

URT. (1974). The wildlife conservation act No. 12 of 1974. United Republic of Tanzania; 1974b.

URT. (1998). Wildlife policy of Tanzania. Dar es Salaam, Tanzania: Ministry of Natural Resources and Tourism.

Wells, M., \& Brandon, K. (1992). People and parks: linking protected area management with local communities. World Bank, World Wildlife Fund and U.S. Agency for International Development, Washington DC. 
Woolf, A., \& Roseberry, J.L. (1998). Deer management: our profession's symbol of success or failure? Wildlife Society Bulletin, 26(3), 515-521. 


\section{LEGAL HUNTING OF WILDLIFE OUTSIDE OF TARANGIRE NATIONAL PARK, TANZANIA}

\section{Introduction}

The Tarangire area is one of the important hunting concessions in Tanzania. It has been divided into at least three hunting blocks and leased to different hunting companies by the Wildlife Division. Legal hunting by both residents and foreign trophy hunters occurs here. There were five hunting camps in the area when this study was underway. However, the number of camps and their locations vary between hunting seasons as a result of choices by the concession owners. The hunting camps are used for short stays by trophy hunters. It is a place where the processing of animals trophies and temporary storage is done. Each hunting camp keeps (on log books) records of all the animals hunted during that hunting season and year at the camp. Hunting log books for previous years may be found at the camps if the camp is old and well established. Records in log books of all shot animals are exactly equal to those on the hunting permits issued by the Wildlife Division.

Although the hunting industry in Tanzania has become more organised and better managed over the past decades, many important aspects remain shrouded in secrecy (Baldus \& Cauldwell, 2004). The Wildlife Division in charge of the hunting is notoriously reluctant to provide hunting statistics lest administrative irregularities be discovered. There is a long chain of bureaucracy a researcher has to go through to get the information. This complicates the data mining process especially when time is limited.

\section{Data collection}

The hunting data for the past four years were obtained through intensive reviewing of the hunting log books stored at the hunting camps and from the offices responsible of hunting in the study area. Between December 2007 and January 2008, I made weekly visits to the hunting camps to determine whether or not hunting had been effected. A record of all the kills found was made on a data sheet, noting the number of each species killed, and where possible, their ages. Because all the camps had their trophies still under the shades being dried, I used these trophies to verify all the kills made 
from the start of the hunting season, i.e. from 1 July. Trophy verification was done by identifying and counting of the trophies available inside the processing shades and temporary stores and cross-checked with the total number that was recorded on the hunting logbooks in that period of time. I used my field experience to identify the trophy species and age for each species namely, impala, Thomson's gazelle, wildebeest and zebra. Additionally, I requested the hunting logbooks of the previous four years, where these were still kept in each of the hunting camps. I extracted all the information on the four species of wildlife that had been hunted between 2003 and 2006 years inclusive. For logbooks not available at the camps, I viewed copies of the permits filed with the respective district game offices.

Data on legal hunting by citizens were obtained from the District game office in charge of issuing hunting licence to citizen hunters. As there were no means to verify these data, I relied on the hunting permits issued and not the quotas that were available for individual species. Issued permits fairly indicate the number and species of animals hunted. Because citizen hunters are allowed a maximum of fourteen days in the field to hunt and obtain their animal, these permits were appropriate sources of data. All citizen hunting data was collected from Monduli, Simanjiro and Babati districts game offices.

\section{Results}

The citizen and tourist hunting statistics are presented in Table 2.0. The mean number of animals hunted by citizens was three to ten-fold that removed by the foreign hunters for most species, except zebra for which there is no legal hunting by citizens. Citizens hunted wildebeest the most followed by impala and Thomson's gazelle. Tourist offtake was high for zebra followed by impala, wildebeest and Thomson's gazelle. Moreover, when the data are combined from both citizen and tourist hunting, wildebeest comes out the most hunted species followed by impala, Thomson gazelle and zebra last. Impala ranks second in every case when the data are examined. There were a decreasing number of animals taken by citizen hunters over the five years; where as the annual hunt was more consistent for trophy hunters (Table 2.0). 
Table 2.0 (a-c): Wildlife killed by citizen and tourist hunters over the five year period 2003-2007 in the study site. All the hunted animals are listed as adult males, as required by the Tanzania hunting policy. * Zebra is not allowed for hunting by citizens under the wildlife law (URT, 1974).

a) Citizen hunting

\begin{tabular}{llllllll}
\hline wildlife species & $\mathbf{2 0 0 7}$ & $\mathbf{2 0 0 6}$ & $\mathbf{2 0 0 5}$ & $\mathbf{2 0 0 4}$ & $\mathbf{2 0 0 3}$ & mean & S.D \\
\hline Zebra* & 0 & 0 & 0 & 0 & 0 & 0 & 0 \\
Impala & 46 & 42 & 90 & 97 & 95 & 74 & 27.5 \\
Thomson's gazelle & 45 & 42 & 65 & 73 & 78 & 60.6 & 16.3 \\
Wildebeest & 71 & 67 & 90 & 95 & 93 & 83.2 & 13.2 \\
\hline
\end{tabular}

b) Trophy (tourist) hunting

\begin{tabular}{llllllll}
\hline wildlife species & $\mathbf{2 0 0 7}$ & $\mathbf{2 0 0 6}$ & $\mathbf{2 0 0 5}$ & $\mathbf{2 0 0 4}$ & $\mathbf{2 0 0 3}$ & mean & S.D \\
\hline Zebra & 27 & 27 & 14 & 21 & 17 & 21.2 & 5.8 \\
Impala & 21 & 14 & 13 & 18 & 23 & 17.8 & 4.3 \\
Thomson's gazelle & 6 & 9 & 4 & 7 & 6 & 6.4 & 1.8 \\
Wildebeest & 24 & 17 & 10 & 10 & 8 & 13.8 & 6.6 \\
\hline
\end{tabular}

c) Combined citizen and trophy hunting

\begin{tabular}{llllllll}
\hline wildlife species & $\mathbf{2 0 0 7}$ & $\mathbf{2 0 0 6}$ & $\mathbf{2 0 0 5}$ & $\mathbf{2 0 0 4}$ & $\mathbf{2 0 0 3}$ & mean & S.D \\
\hline Zebra & 27 & 27 & 14 & 21 & 17 & 21.2 & 5.8 \\
Impala & 67 & 56 & 103 & 115 & 118 & 91.8 & 28.5 \\
Thomson's gazelle & 51 & 51 & 69 & 80 & 84 & 67 & 15.6 \\
Wildebeest & 95 & 84 & 100 & 105 & 101 & 97 & 8.1 \\
\hline
\end{tabular}

\section{Discussion}

The trend of legal hunting shows an overall decline over five years. This is due entirely to a reduction in citizen hunting. This suggests a reduced size of quotas being set by the Wildlife Division probably with a desire to maintain tourist income. The reduction of quotas may also be a response to decreasing populations of hunted animals as it has been observed in recent studies (Caro et al., 1998b; Stoner et al., 2007).

The rate at which the citizen hunting removes wildlife is higher than is taken in trophy hunting, presumably due to increasing demands for wild meat from the burgeoning human population. There has been a $4.0 \%$ increase in human population over ten 
years in the Arusha, Manyara and Dodoma regions surrounding the study area (URT, 2002). As the cost of buying one wild animal to hunt is low, it is probably more affordable even for an average person. For example, the current price for buying one buffalo to hunt for citizens is 42,000/= Tanzania Shillings (=36 USD), less or similar to the price of sheep or big goat at Tanzania local market. This may be easier for some relatively affluent citizens to go for hunting.

There is no hunting of zebra by citizens. This does not mean that zebra's meat is not edible. In essence zebras' meat is equally consumed in several local communities in Tanzania. For example, a study of consumption patterns of game meat among local communities near the Serengeti national park, (Ndibalema \& Songorwa, 2008) found that zebra is preferred to other animals for its meat. This preference was based on the meat taste and the economic gains from selling meat in the locality. From the above study, it can be suggested that if zebra were allowed to be hunted legally by citizens, the hunting statistics would be higher.

Only adult male animals are hunted. However, due to less supervision of the hunting process in the field (especially for citizen hunting), it is difficult to ascertain if all the hunted animals were males as there were no means to verify citizen hunting data. My own field experience show that most citizen hunters do not comply with the rules on the hunting permits thereby hunting more than is indicated, hunting females or species not indicated on the permits (personal observations, 2004-2006). Even trophy hunters sometimes hunt females especially in species difficult to sex (Spong et al., 2000)

Visual determination of exact age of the animals killed was difficult. This is because kills from citizen hunting could not be viewed. Even the trophies found at the hunting camps gave little information about the age of the animal hunted and I had no ability to examine teeth. Therefore I regard all the hunted animals as adult males as required by the hunting policy.

Finally, the hunting statistics above may be lower than the actual legal offtake if crippling losses and unreported kills were documented. Perhaps this would increase the kill to twice that reported here. But for the purpose of this study the available actual data is appropriate for doing population viability analyses. 


\section{Chapter summary}

This chapter documents the legal kill of four species of wild game animals around the Tarangire National Park study area during 2003-2007, as determined from hunting permits issued from offices managing the local hunting industry. The mean annual legal kill was 21, 92, 67 and 97 zebra, impala, Thomson's gazelle and wildebeest respectively. A short description of the trend of the data is given. Data from this chapter will be used in the final assessment of the impacts of hunting as presented in Chapter six.

\section{References}

Baldus, R.D., \& Cauldwell, A.E. (2004). Tourist hunting and its role in development of wildlife management areas in Tanzania. $\underline{\text { ww.cic- }}$ wildlife.org/uploads/media/Hunting_Tourism.pdf (accessed on 29, July 2008).

Caro, T.M.,Pelkey, N.,Borner, M.,Severre, E.L.M.,Campbell, K.L.I., et al. (1998b). The impact of tourist hunting on large mammals in Tanzania: an initial assessment. African Journal of Ecology, 36(4), 321-346.

Ndibalema, V.G., \& Songorwa, A.N. (2008). Illegal meat hunting in serengeti: dynamics in consumption and preferences. African Journal of Ecology, 46(3), 311-319.

Spong, G.,Hellborg, L., \& Creel, S. (2000). Sex ratio of leopards taken in trophy hunting: genetic data from Tanzania. Conservation Genetics, 1(2), 169-171.

Stoner, C.,Caro, T.,Mduma, S.,Mlingwa, C.,Sabuni, G., et al. (2007). Changes in large herbivore populations across large areas of Tanzania. African Journal of Ecology, 45(2), 202-215.

URT. (1974). The wildlife conservation act No. 12 of 1974. United Republic of Tanzania; 1974b.

URT. (2002). Population and housing census of 2002 in Tanzania. http://www.tanzania.go.tz/census/reports.htm. Accessed on 3/8/2008. 


\title{
3. POACHING OUTSIDE TARANGIRE NATIONAL PARK, TANZANIA: ESTIMATING ILLEGAL HUNTING OF WILDLIFE USING THE RANDOM RESPONSE TECHNIQUE
}

\begin{abstract}
Determining illegal resource extraction is difficult because people seldom tell the truth about illegal actions for fear of incrimination. I used the Randomised Response Technique (RRT) to estimate illegal hunting of four wildlife species (zebra, impala, wildebeest, Thompson's gazelle) by the Masai community from areas outside Tarangire National Park, Tanzania. The Masai traditionally do not to eat wild meat, but often assist others to hunt wild animals. I adapted the RRT by asking each of 298 household interviewees whether they ever had killed or witnessed someone killing animals and established a likely illegal kill. Results show that the illegal offtake of the four species is up to $4 \mathrm{x}$ higher than the legal offtake. Despite their tradition, circumstantial evidence shows that the Masai do kill the wildlife on their farms and are involved in poaching of the wildlife for meat. Further, the wildlife-Masai conflicts are on the rise and they receive little benefit from the wildlife close to them. Unemployment and insufficient benefits from the wildlife were significantly positively correlated with levels of illegal hunting. Definitive management schemes that provide realistic benefits to the Masai need an emphasis to ensure any conservation prospect for wildlife in the area.
\end{abstract}

Keywords: Tarangire National Park, poaching, Masai, Random response technique,

\section{Introduction}

Illegal use of biological resources is increasingly recognised as a significant threat to the sustainability of biodiversity in many ecosystems (Albers \& Grinspoon, 1997; Brashares et al., 2004; Dublin et al., 1990). In the western and central Africa, poaching is driving several species to local extinction (Fischer \& Linsenmair, 2001; Newby, 1990; Yamagiwa, 2003). It has also largely reduced the populations of savannah mammals in many reserves throughout eastern and south Africa (Dublin et al., 1990; Leader-Williams et al., 1990; Mann, 1995). 
Although Tanzania is renowned for its protected areas and conservation commitment, its wildlife species and their habitats are increasingly threatened. Thirty-eight animal species have gone extinct in Tanzania; a rate second only to South Africa amongst African countries. Tanzania also has the most threatened mammal species (IUCN, 2007). This problem is due partly to over use of wildlife by people and the country's limited conservation resources. An increasing human population near reserves put pressures on these resources. In the western corridor of Serengeti National Park, for example, intensive poaching has been highest around densely populated areas (Holmern et al., 2002; Loibooki et al., 2002; Thirgood et al., 2004). As a consequence of the increased poaching in national parks and adjacent areas (Hofer et al., 1996), the population density of the wildlife has declined throughout the country (Caro et al., 1998; Stoner et al., 2007).

Many methods to evaluate poaching have been used e.g. observing physical evidence left by poachers or interviews with the natural resource guards (Holmern et al., 2002; Leader-Williams et al., 1990); records of poachers arrested along with measuring of patrol efforts (Loibooki et al., 2002; Wright et al., 2000); and comparison of stocks of biological resources over time (Brashares et al., 2004; Stoner et al., 2007).

These methods all have limitations. It is difficult to source information on illegal activities that are under-reported or unwitnessed. Poaching in reserves occurs during the day and night (Arcese et al., 1995) and so most of these actions go undetected by the resource guards. The methods mostly rely on resource guards or administrators who have less information on illegal activities than the local people who are the poachers, traders or consumers of wildlife. Additionally when resource users are asked directly they are highly likely to refuse to answer or to give untruthful answers. In direct surveys, the frequency of illegal activities is under reported. Information that is needed for effective decision making is always missed when these methods are used to inquire about threatening or sensitive issues like poaching.

It has been shown elsewhere that a random response technique (RRT); (Greenberg et al., 1969; Warner, 1965) performs well in surveys of incriminating issues. This technique has been widely applied in social studies to quantify incidences of rape, tax evasion and induced abortion (Houston \& Tran, 2001; I-Cheng et al., 1972; Soeken \& 
Damrosch, 1986; Tezcan \& Omran, 1981). It also holds promise for researching illegal resource use (Chaloupka, 1985; Kline \& Schill, 1995; Solomon et al., 2007; Wright, 1980). This study adapts the RRT to estimate the level of poaching of four ungulate species outside of Tarangire National Park, Tanzania. The goal of this study was to estimate the number of the four animal species; impala (Aepyceros melampus Matschie), Thomson's gazelle(Gazella thomsonii Günther), wildebeest (Connochaetes taurinus Thomas) and zebra (Equus burchellii Matschie) (Appendix 3.0) that have been killed by poachers from the area and use these data to examine the impacts of hunting (see Chapter 6). To my knowledge there has been no research using the RRT to quantify wildlife poaching in areas afforded less or no protection and yet they are important reservoirs of the wildlife in Tanzania. This study aids an understanding of the extent of the problem with a view to providing information appropriate for management and evaluation of current conservation strategies in similar areas.

\section{Use of Randomised Response Technique in Surveys of Sensitive Issues}

The RRT, first proposed by Stanley Warner (Warner, 1965) is meant to increase trust and therefore encourage cooperation from respondents when asking incriminating questions.

The technique uses two questions, one sensitive and another non-sensitive. In his initial model, Warner asked two related questions such as 'I am a member of sensitive behaviour A' and 'I am not a member of sensitive behaviour A' to assess the frequency of the sensitive behaviour A. Either of these questions is answered as a result of rolling a randomising device which directs respondents to answer one question with a 'yes' or 'no'. A maximum privacy is required for respondents when rolling a randomising device. As the interviewer is not allowed to witness the die roll and so the researcher does not know which question the respondent is answering. Thus, the 'yes' or 'no' answer is essentially known only by the dice roller. In this way respondents build trust from which truthful answers are given to the questions being asked. Nevertheless, following probability rules, there is a known probability of choosing a sensitive question when rolling a die. Using this probability and the proportion of 'yes' answers from the nonsenstitive question, and the frequency of all the observed yes answers from a set of the two questions asked, the researcher can 
estimate the proportion of the surveyed population who are involved in the sensitive behaviour A.

Since its inception several modifications and improvements on Warner's model have occurred with a view to increase its ability to protect respondents' privacy and encourage truthful answers (see review by Fox \& Tracy 1986). These modifications hinged on statistical developments and the nature of the questions being asked (Greenberg et al., 1969). The two 'related questions' previously used were replaced by the 'unrelated questions' such that 'Do you have sensitive attribute A?' and 'Do you have nonsenstitive attribute B?' This model requires two independent random samples. In the first sample, respondents are asked to answer a sensitive question with a probability of $(P)$ and that of unrelated question (1-P) following outcomes of a randomising device. In the second sample the probabilities of responding to the two questions are reversed. Using data from the two random samples the proportion of a sensitive attribute can be estimated (Greenberg et al., 1969). Subsequent development of this model resulted in use of a second nonsensitive behaviour whose probability is already known a priori according to the randomising device. Consequently, this model requires a single random sample of respondents (Greenberg et al., 1969).

The application and verification studies of an unrelated question model have spawned a wide range of fields. In a review (Umesh \& Peterson, 1991) and validation study (Lensvelt-Mulders et al., 2005), the authors show that sensitive behaviours are admitted to more often when this method is used over direct questioning. In surveys of induced abortion, Tezcan \& Omran (1981) and Lara et al. (2004) report estimates that were higher than those obtained through direct questioning. In another study of tax evasion in Australia, Houston \& Tran (2001) used the unrelated question design to estimate the incidence and type of income tax evasion. They too report higher estimates of people evading tax with the RRT method relative to the direct questioning model. Moreover, in studies of fraud (Landsheer et al., 1999) and rape (Soeken \& Damrosch, 1986), the randomised response method has proved to be useful in revealing rates of these behaviours. The application of RRT and its results are based on the premise that respondents are willing to participate. Demographic variables such as education level, gender and age of respondents have been observed to significantly correlate with successfully using the RRT (I-Cheng et al., 1972; Lara 
et al., 2004; Soeken \& Damrosch, 1986; Tezcan \& Omran, 1981; Umesh \& Peterson, 1991).

\section{Application of RRT in the field of natural resources conservation}

Advanced attempts to manage exploited biological resources can be flawed without estimates of illegal harvest of the resources (Smith et al., 1989). Due to the paucity of information on poaching and the burgeoning threats on the biological resources, the randomised response technique is increasingly being used. Wright (1980) used the unrelated question model to estimate deer poaching in Iowa. The number of Iowa deer poached by farmers alone was about equal to the legal offtake.

Smith (1989) used the RRT to estimate the prevalence of fishing without licence in Colorado. This study estimated that $22 \%$ of respondents had at least fished once without a licence in the previous year. In a study of noncompliance with angling regulation on three Idaho waters Kline et al. (1995) estimate about 29\% noncompliance with barbless hook regulations. The authors concluded that RRT is a viable tool for estimating rates of angler noncompliance with regulations. In another separate study to assess permit noncompliance in the collection of shells from a marine park in Australia, Chaloupka (1985) used a Table of random numbers and two questions with different probabilities of being selected. This study reported a significant noncompliance with permit rules. Similarly, in Kibale National Park Uganda, Solomon et al (2007) report higher rates of illegal use of various natural resources from the park compared to those estimated by the direct inquiry of the illegal use.

Although, the literature shows an astounding promise for RRT in surveys of illegal resource exploitation, only a few studies have been attempted in the field of biological conservation. Several reasons have been suggested for this including that the method itself thus difficult for the average person to comprehend (Smith, 1989). The complex mathematical derivations and other statistical jargon in published papers on this method (Greenberg et al., 1969) sometimes look unappealing to an average researcher. Also, the difficulty found by researchers explaining the technique to respondents perhaps, make this technique a little more difficult for biologists to 
assimilate easily (Kline \& Schill, 1995). Nevertheless, the RRT remains a useful tool under the current conservation crisis.

\section{Methods}

\section{Study site}

This study was done in four village communities in Masailand adjacent to Tarangire National Park. The Masailand forms part of non-protected areas and game controlled areas, Lolkisale and Simanjiro (Fig. 3.0). Its significance as dispersal and calving range for migrant wildlife makes it the heartland of the Tarangire National Park (Kahurananga, 1981). Wildlife species seasonally migrating into this area include but are not limited to zebra, wildebeest, buffalo (Syncerus caffer Sparrman), eland (Taurotragus oryx Lydekker), and elephants (Loxodonta Africana Matschie). These animals spend more than half the year grazing in this area. Resident game animals are impala, giraffe (Giraffa camelopardalis Matschie), lesser and greater kudu (Tragelaphus species Heller), Thomson's gazelle, Grant's gazelle (Gazella granti Brooke) and warthogs (Phacochoerus aethiopicus Cretzschmar). The area is of semiarid savannah vegetation with low rainfall regimes of between 450-600mm per year (Kahurananga, 1979). This area is also used by the agro-pastoralist Masai communities who have long shared the ranges resources with wildlife. Formal licensed hunting is conducted each year in the area during the hunting season (JulyDecember). There is no formal government body in the field overseeing protection of wildlife, a situation that allows illegal hunting, particularly during the period when sanctioned hunting is not occurring.

\section{The communities}

The local inhabitants are mainly Masai who were traditionally nomadic herders that rarely ate bush meat. They largely depend on range resources, particularly grazing pastures for their livestock (Nelson, 2000). In the past two decades rapid human population growth and increased demand for livelihood have caused unsurpassed resource depletion in the Masailand (Galaty, 1981; Mwalyosi, 1991). As a consequence, traditional nomadic pastoralists have opted for a more permanent settlement (Mwalyosi, 1992). 
The Masai still build traditional mud-dung houses arranged in a circle around a cattle corral, a kraal or boma as it is commonly known. A kraal is a polygynous family compound, (thus a household) and consists of a large circular thornbush fence around a ring of houses ranging from 4-8 huts (Gulliver, 1969). The household may consist of an elderly father, his wives and his married sons. Kraals can be distinguished by the distance between them as they tend to be widely dispersed. There is a very organized social grouping system amongst the Masai community with the family activities categorised according to the age groups. They move up through a hierarchy of grades, including those of warriors, junior and senior elders. The warriors (men between 1735 years of age) are in charge of the society's security, and spend most of their time now on walkabouts throughout the Masailand (Galaty, 1982). Together with boys they herd the livestock. Women are responsible for making the houses as well as supplying water, collecting firewood, milking cattle and cooking for the family. Elders carry out duties as advisors and attend to family problems such as diseases and are sometimes involved in trading cattle.

\section{Sampling Procedure}

This study used a randomised response method and a questionnaire survey. A sample of 298 households taken from four systematically sampled villages; Lolkisale ( $n=93)$, Sukuro $(n=71)$, Terrat $(n=88)$, and Kimotorok $(n=46)$ were used. These villages were chosen due to their location in close vicinity to each other, because they had historically been sharing rangelands with wildlife, they had high poaching incidences relative to other villages (TNPR, 2006) and, had land-use developments particularly agricultural fields around them.

Each village has several sub-villages (wards) with a village centre where there is a village administrative office and other social amenities. Sub-villages located on the outskirts are politically administered by sub-village heads that are responsible to an overall village chairman. Within Masailand, these sub-villages whose kraals may range from 10-25 average in size (Huntingford, 1953), are highly dispersed probably to maximize use of range resources for their herds. For this study, I sought to survey all households in all sub-villages but excluding the central village because they are more distant from wildlife. 
Having obtained a written permit from the village head, we went to each sub-village head who assigned us another man who took us through each kraal for the survey. This was important because Masai people would rarely disclose any information to foreigners they do not know. Also having their fellow Masai man with us, built a sense of trust for the survey and enabled explanations in the respondents' local language when needed. Apart from this, we also had a contracted fellow Masai with whom we worked in all villages throughout the survey. These factors minimised bias from intentional falsification of answers or refusal to respond (Umesh \& Peterson, 1991). We walked between household kraals and sometimes used a car to shuttle between sub-villages when the distance was not easily walkable.

For the RRT process, I used one respondent from each household. These individuals were those who had appropriate knowledge of the household affairs and sound experience in living close to the wildlife. Thus, respondents were above 18 years of age and mostly elder sons of the household heads and whose major role was herding and protecting livestock. With these criteria, all household elders and women, unless otherwise stated, were eliminated from doing the RRT process. Nevertheless, there were cases where the targeted individuals were rejected due to not understanding the protocol or possibly fears. These were replaced by their brothers in the same boma. Questionnaires were completed by the majority of household members including the head. All the interviews were conducted in Kiswahili, a national language of Tanzania.

\section{Administering the Randomised Response Technique and the Questionnaire survey}

Illegal killing of the four ungulate species namely; zebra, impala, wildebeest and Thomson's gazelle was estimated over a three year period between 2005 and 2007 inclusive. To better explain the RRT responses, I asked four questions about;

(i) The negative impacts and the benefits that Masai communities obtain by living in the vicinity of the wildlife.

(ii) Whether they get any allowances from the government or companies conducting hunting of wildlife from around the study site.

(iii) Whether any member of the household is employed in any wildlife sector in the area. 
(iv) Their opinion on the law protecting wildlife in the area.

These questions are relevant to assessing the individuals' willingness to protect and conserve the wildlife. I examined the linkage between the RRT responses and the costs and benefits of wildlife to the local communities in the area.

Surveying illiterate and traditionally conservative community such as the Masai poses challenges. This community does not traditionally eat wildlife meat. Because of this they have been allowed by the governments to live within wildlife rangelands throughout east Africa for decades. The majority of areas that the Masai occupy are also wildlife areas not protected by the strict rules that apply to the National Parks. There is, nevertheless, illegal hunting of game animals by people from within and outside the Masai community. Controlling poaching in these areas is difficult without any information at hand. A great deal of information on illegal hunting, however, rests with the Masai.

I piloted the RRT method in 42 households in a Masai community near Tarangire National Park. I used the unrelated question design 'Did you hunt this animal without permission in the past three years since, 2005?' The second question was, 'Did you see head when you flipped the coin?' I administered the RRT process using a New Zealand 50 cent coin and photographs of the named wild ungulates as described in Solomon et al. (2007). The primary sample size targeted in the pilot was equivalent to an average size of a Masai village.

In the pilot study twelve households refused to participate in the survey on the grounds that they do not hunt wildlife. The remaining sample $(n=30)$ surprisingly gave negative answers to both of the questions. From these responses, it was not possible to estimate the levels of poaching as there was no "yes" response to either of the questions asked. All respondents demonstrated an understanding of the RRT process, but I suspected the following to have caused this outcome. The fact that the Masai do not traditionally eat wild meat probably could have caused this nonresponse. My field experience (personal observation, 2004-2006) and a discussion I carried out with the Masai men showed that the Masai hunt and eat wild meat during seasons of droughts and famine. They also kill the wildlife that feed on their crops 
(TNPR, 2006). Since rain is highly erratic and droughts are frequent, regular shortage of food for this human population is likely. Fear and the resulting consequence of disclosing the poaching issues also were suspected to make the Masai refuse. Another possible reason for non-response was that, perhaps the Masai were involved in illegal hunting of wildlife. The outcomes of the pilot survey did not worry me because even a sub-tribe of Masai, Dorobo (Huntingford, 1955; Zwanenberg, 1976) who live off land (by hunting and gathering) denied hunting wildlife when they were asked this question.

In the study area there are no wildlife protection guards, nor is there any person or body from which I could get data on the illegal hunting. Under this situation, the only people to get this information from were the inhabitants of the area, the Masai. Thus I decided to modify the wording in the sensitive question to encourage cooperation and truthful answers from the respondents. The sensitive question was reworded into "Did you hunt or seen any one person hunting this wild animal out of hunting season since the year 2005?" The non-sensitive question was 'Did you see head when you flipped the coin? The RRT procedure was described and demonstrated before each respondent using a dollar coin, its photo and photographs of the named animals as in Solomon et al. (2007). I explained the purpose of our visit on arrival at each household compound by requesting the household members to provide information to a student undertaking nature conservation studies in New Zealand. By emphasising that our survey was absolutely for schooling purpose, we minimised response bias from respondents on their feeling of the potential outcomes of their responses. Interviews were conducted in Kiswahili language and any raised questions were answered. I made clarifications on the sensitive question that hunting could have included killing or helping other people to kill wildlife for any reason, be it for subsistence meat, commercial, or accidentally killed in farms as a results of raiding crops.

Having respondents who understood the protocol, they independently tossed up the coin and answered 'yes' or 'no' to either of the above questions. Answers for each coin toss were recorded and thereafter a questionnaire survey was completed. 
The questions were mostly close-ended, where respondents were presented with response alternatives (Appendix 3.1). A few questions however were open-ended for respondents to give their own views. I pre-tested the structured questionnaire alongside the RRT method; only slight adjustments were made to it. Several questions were posed including, identifying key threats from the wildlife; the wildlife related benefits accrued, major economic activities of the people, their employment status in the wildlife industry in the area, and their opinion on the law protecting wildlife in the area particularly on the hunting perspective.

The other questions asked were for respondents to comment on the population trend of wildlife being investigated (also presented on photographs) since the time they lived in the area, residence time and why people would like to poach the wildlife. Overall, these questions were used to gauge people's attitude over the wildlife in the area. On the questionnaire I recorded age and education level of respondents.

\section{Data Analysis}

The data were analysed using two methods. The proportion of each of the wildlife species that have been killed illegally (without permission) from this area was estimated using the formula below (Fox \& Tracy, 1986)

$R=Y-D(1-P) / P \quad$ with a variance, $\operatorname{Var}(R)=Y(1-Y) / n P^{2}$

Where

- $R=$ estimation of the proportion of respondents who had ever poached or seen any person killing the wildlife species in question.

- $Y=$ observed proportion of respondents who answered 'yes' on the RRT survey portion.

- $D=$ proportion of the population expected to have responded 'yes' to the nonsensitive item of RRT (i.e. coin head)

- $P=$ probability of responding to the sensitive behaviour question, as there were two choices, for a coin head or photo of an animal from a bag $(p=0.5)$

- $\mathrm{n}=$ sample size 
Notes: The probability of having 'yes' answers to the innocuous question (did you see head of a coin) is essentially 0.0625 (from $0.5^{*} 0.5^{*} 0.25$ ) because the first toss of a coin is independent of picking photo of a coin head from the bag. All the 'yes' answers to the non-sensitive question were therefore given by $0.0625 * \mathrm{~N}$, where $\mathrm{N}$ denotes total number of respondents. This probability was then used to obtain the proportion of the population expected to have positively responded to the nonsensitive question (D)

Information from the questionnaires for all the study villages was used to quantify the proportion of households whose income is primarily from agricultural farming (i.e. crop cultivation and livestock keeping). This proportion was multiplied by the total human population size $(n=16652)$ from recently published census of the area (URT, 2002), to obtain a total number of people $(n=16202)$ involved in agricultural activities in the study villages (Table 3.2). Using 11.2 average persons per household calculated by Mwalyosi (1991) for the area, the number of people involved in agricultural activities $(n=16202)$ was divided by the average number of people in a household to get a total number of households in the villages whose income is primarily from agricultural farming.

Following Wright's (1980) procedure for estimating the number of deer poached by farmers in Iowa, together with the method of Holmern et al. (2002) for deriving the number of local residents involved in hunting around the Serengeti National Park, the proportion estimates of the household respondents involved in illegal hunting of the wildlife in Masailand (hereafter named, $R$ - estimate in Table 3.0) was multiplied by the total number of farming households to derive the total number of households involved in illegal hunting of the animal species in question. On the assumption that each household could have caused death to at least one animal through illegal killing over the course of three years on which this study asked, I derived the average estimates of the animals killed in one year as shown in Table 3.1.

The analysis of the questionnaire data was done using MINITAB release 13.32. 


\section{Results}

\section{Poaching estimates of the wild ungulates}

The estimated proportions $(R)$ of the respondents who answered yes to the hunting question are shown in Table 3.0. Kruskal-Wallis tests show that there is no significance difference in the proportions of poaching between the four villages $(\mathrm{H}=$ 4.53 $\mathrm{DF}=3, P=0.210)$ as well as across the four species $(\mathrm{H}=2.45 \mathrm{DF}=3, P=0.485)$. The proportion of the zebra hunted is high at around Terrat and Kimotorok villages ( $R=0.56$ and $R=0.42$, respectively) and appears to be the third most hunted animal on a pooled data. Impala is overall, the most heavily hunted species with the poaching estimates being larger at around Lolkisale and Terrat villages $(R=0.59$ and $R=0.44)$ than in other villages. Of all the species, wildebeest is the least poached animal ( $R=0.37$ ). However, wildebeest is poached in relatively large numbers at around Lolkisale village $(R=0.42)$. Thomson's gazelle is most hunted around Terrat $(R=0.51)$ and Sukuro $(R=0.48)$ village communities.

The estimated median number of animals that have been illegally killed is significantly different from the legal hunt of the game species in the area (MannWhitney $\mathrm{W}=26.0, P=0.030$ ). Table 3.1 shows estimates of animals illegally killed in comparison to those killed by licensed hunters in the area as determined from the number of issued hunting permits (Chapter 2)

Table 3.0: Estimated poaching levels of four wildlife species in Masailand, Tanzania indicated by the proportions of respondents $(R)$ answering "yes" to the hunting question.

Wildlife species investigated

\begin{tabular}{|c|c|c|c|c|c|c|c|c|}
\hline \multirow[b]{2}{*}{$\begin{array}{l}\text { Village name } \\
\text { (sample size) }\end{array}$} & \multicolumn{2}{|l|}{ Impala } & \multicolumn{2}{|c|}{ Thomson's gazelle } & \multicolumn{2}{|c|}{ Wildebeest } & \multicolumn{2}{|l|}{ Zebra } \\
\hline & $\begin{array}{l}R \text { - } \\
\text { estimate }\end{array}$ & $\begin{array}{l}\text { Variance } \\
\text { of } R\end{array}$ & $\begin{array}{l}R \text { - } \\
\text { estimate }\end{array}$ & $\begin{array}{l}\text { Variance } \\
\text { of } R\end{array}$ & $\begin{array}{l}R \text { - } \\
\text { estimate }\end{array}$ & $\begin{array}{l}\text { Variance } \\
\text { of } R\end{array}$ & $\begin{array}{l}R \text { - } \\
\text { estimate }\end{array}$ & $\begin{array}{l}\text { Variance } \\
\text { of } R\end{array}$ \\
\hline Lolkisale (93) & 0.447 & 0.011 & 0.285 & 0.011 & 0.421 & 0.011 & 0.299 & 0.011 \\
\hline Sukuro (71) & 0.401 & 0.014 & 0.485 & 0.013 & 0.348 & 0.014 & 0.293 & 0.013 \\
\hline Kimotorok (46) & 0.428 & 0.021 & 0.347 & 0.021 & 0.375 & 0.021 & 0.428 & 0.021 \\
\hline Terrat (88) & 0.59 & 0.009 & 0.511 & 0.01 & 0.36 & 0.011 & 0.564 & 0.01 \\
\hline Combined & 0.476 & 0.003 & 0.412 & 0.003 & 0.379 & 0.003 & 0.399 & 0.003 \\
\hline
\end{tabular}


Table 3.1: Estimated numbers of animals killed from the study area by illegal hunters on average in one year in comparison to legal offtake. For derivation of the estimates and village farmer households see the text.

\begin{tabular}{|c|c|c|c|c|}
\hline \multirow{2}{*}{$\begin{array}{l}\text { Village size } \\
\text { (2002 census) }\end{array}$} & \multirow{2}{*}{$\begin{array}{l}\text { Average farmer } \\
\text { households }\left(=f^{*} n\right)\end{array}$} & \multirow[b]{2}{*}{ Species } & \multicolumn{2}{|c|}{ Hunt estimates } \\
\hline & & & illegal & legal \\
\hline $\begin{array}{l}\text { Lolkisale } \\
(\mathrm{n}=6427)\end{array}$ & 6253.471 & Zebra & 187.7 & 23 \\
\hline $\begin{array}{l}\text { Sukuro } \\
(n=4470)\end{array}$ & 4349.31 & Wildebeest & 178.1 & 93 \\
\hline $\begin{array}{l}\text { Kimotorok } \\
(\mathrm{n}=368)\end{array}$ & 358.064 & $\begin{array}{l}\text { Thomson's } \\
\text { gazelle }\end{array}$ & 1973 & 57 \\
\hline $\begin{array}{l}\text { Terrat } \\
(n=5387)\end{array}$ & 5241.551 & Impala & 226.2 & 75 \\
\hline Household total & 16202.396 & & & \\
\hline
\end{tabular}

$f=0.973$, a proportional estimate of the households whose income is primarily from agricultural activities, $R=$ proportional estimates of the households involved in illegal killing of wildlife (Table1); $y=11.2$, average number of people in a household, $n=$ village population size

\section{Respondent's demographics}

The majority of respondents have lived within the study site for a long time. Many $(43.2 \%)$ have lived there for $>20$ years, $33.6 \%$ lived in the area for $10-20$ years, and about $23.2 \%<10$ years at most. Most respondents $(77.6 \%)$ had at least a primary level education and the remainder had no formal education at all. All respondents managed to do the RRT exercise successfully.

This community lives on both livestock keeping and subsistence farming. A significant majority $(76.5 \%)$ of respondents were agro-pastorals, $20.8 \%$ purely crop growers and only $2.6 \%$ were engaged in small business such as mini-shops in the Masailand. The young age class (18-25 years old) responded significantly more to the illegal hunting question than the mid-age (25-45) and old (>45 years) group $(\mathrm{H}=8.77$, $D F=2, P=0.012$ ). The poaching proportions reported were not significantly different between respondents with different level of literacy $(\mathrm{H}=2.22, D F=2, P=0.330)$.

\section{The magnitude of wildlife threats to the local people}

When asked of the threats posed by the wildlife to their livelihoods, a range of direct threats were pointed out (Table 3.2). Crop raiding, disease transmission (malignant 
catarrh fever) from wildebeest to cattle, and lion predation on the livestock were of much concern. Some respondents expressed these concerns with anger stating that they would be happy if all wildebeests were exterminated, leaving alive only a few animals which could cause little or no harm to their livestock. The levels of wildlife problems inflicted to the Masai were not significantly different across the four villages $(\mathrm{H}=3.0 \mathrm{DF}=3, P=0.392)$. Despite this however, I found no significant correlation between the threats posed to the Masai by the animals (crop raiding and disease transmission) and level of poaching of the animals in the area $\left(r_{s 4}=0.632, P>\right.$ 0.05). There was also an insignificant negative correlation between all the types of wildlife problems and level of poaching reported in the area $\left(r_{s 4}=-0.40, P>0.05\right)$.

Table 3.2: The percentage of wildlife threats reported by the local people and the number of respondents (in brackets)

Threats from wildlife identified by respondents

\begin{tabular}{llllllll}
\hline & & crop & $\begin{array}{l}\text { Disease } \\
\text { transmission }\end{array}$ & $\begin{array}{l}\text { Crop raid and } \\
\text { disease } \\
\text { transmission }\end{array}$ & All & $\begin{array}{l}\text { No bad } \\
\text { effect }\end{array}$ \\
\hline Sukuro & pasture & raid & $29.0(25)$ & $28.6(2)$ & $24.8(25)$ & $18.4(18)$ & 0 \\
Kimotorok & 0 & $15.1(13)$ & 0 & $20.8(21)$ & $9.2(9)$ & $60.0(3)$ \\
Lolkisale & 0 & $30.2(26)$ & $42.9(3)$ & $23.8(24)$ & $41.8(41)$ & 0 \\
Terrat & $100(1)$ & $25.6(22)$ & $28.6(2)$ & $30.7(31)$ & $30.6(30)$ & $40.0(2)$ \\
\hline
\end{tabular}

\section{The benefits of wildlife resources to the local human population}

Respondents gave different views on the question of direct benefits achieved from the wildlife around them. About 51\% $(\mathrm{N}=298)$ of the locals claimed not to realise any benefits from the wildlife resources. Forty-eight percent credited the neighbouring Tarangire National Park with helping, mentioning social services such school classrooms, cattle trenches, and health dispensaries. A few people (1\%) admitted to enjoying the wildlife meat when given by legal hunters. The realised benefits from the wildlife were significantly different across the villages surveyed $\left(\chi^{2}=18.49, P<0.000\right.$, $D F=3)$.

Because employment and direct monetary benefit from the wildlife are increasingly cherished as incentives for shaping ones' attitude towards the wildlife (Kideghesho et 
$a l ., 2007)$, these questions were left asked. A total of $84.5 \%$ household respondents were not employed and $69 \%$ claimed to receive no funding from the government derived from hunting the animals around the area (Table 3.3). Unemployment and lack of monetary benefits from wildlife are significantly higher and were the top concerns across the villages (Unemployment: $\chi^{2}=20.26, P<0.000, D F=3$; Monetary benefits: $\left.\chi^{2}=20.26, P<0.000, D F=3\right)$. There was a significant positive correlation between the poaching levels and unemployment $\left(r_{s 4}=1.0, P<0.05\right)$ and an insignificant positive correlation between poaching of animals and insufficient monetary benefits as well as realised or tangible benefits in the area $\left(r_{s 4}=0.40, P>\right.$ $0.05)$.

Table 3.3 Respondents' yes (no) answers $(N=298)$ on the question of whether or not they get direct benefit from the wildlife, whether or not were employed in the wildlife business, or get allowance as wildlife benefits from the government or hunting companies around the area. Figures are actual proportions of answers from village respondents relative to total sample size $(N)$

\section{The benefits from the wildlife}

\begin{tabular}{llll}
\cline { 2 - 4 } Villages & Realised or tangible benefits & Employment & wildlife allowance \\
\hline Sukuro & $\mathbf{6 . 7}(17.1)$ & $\mathbf{3 . 4}(20.4)$ & $\mathbf{2 . 3 ( 2 1 . 5 )}$ \\
Kimotorok & $\mathbf{7 . 0}(8.3)$ & $\mathbf{1 . 3}(14.1)$ & $\mathbf{5 . 7}(9.7)$ \\
Lolkisale & $\mathbf{1 7 . 8}(13.4)$ & $\mathbf{3 . 7}(27.5)$ & $\mathbf{1 2 . 7}(18.5)$ \\
Terrat & $\mathbf{1 7 . 4}(12.1)$ & $\mathbf{7 . 0}(22.5)$ & $\mathbf{1 0 . 1}(19.5)$ \\
\hline
\end{tabular}

\section{Local peoples' opinions on the trend of wildlife population and hunting in the} area

I asked the Masai how the population of wildlife has been fairing in the study area. Across the four villages, the majority $(41.9 \%)$ of respondents felt that the wildlife have been increasing $\left(\chi^{2}=17.3, F D=3, p<0.001\right)$, and $33.5 \%$ said it was decreasing. Others $(9 \%)$ claimed the population of herbivores to be sTable and associate this with the seasonal migration from and to Tarangire National Park, and $15.4 \%$ of respondents had no idea.

When asked why they think people would illegally kill wildlife around the area, many reasons were given. A combination of commercial selling, local bushmeat consumption and retaliatory killing (revenge killing of animals that have destroyed 
crops or livestock), were stressed (Table 3.4a). Twenty six percent said illegal hunting is done primarily for selling bushmeat, $8.7 \%$ for subsistence and $3.7 \%$ said animal deaths were a result of retaliation killing. In one village community (Lolkisale) some respondents adamantly admitted to illegally trade on the wild meat. They argued "the wildlife is causing great damage to our crops and livestock while these resources seem to benefit only a few people in this country". They added "where is our right? We are losing our wealth to these animals, no compensations and only to be told to humble with this beast!" 'This is the only way we can bring back our lost capital' another person reported.

Table 3.4a: Respondents' opinions in percentage, \% (n) on why people would tend to kill the wildlife from around the area.

\begin{tabular}{lllllll}
\cline { 2 - 6 } & \multicolumn{7}{l}{ Reasons cited for illegal hunting } & & & \\
\cline { 2 - 7 } Villages & $\begin{array}{l}\text { For } \\
\text { bushmeat }\end{array}$ & $\begin{array}{l}\text { poach } \\
\text { for selling }\end{array}$ & $\begin{array}{l}\text { easy to } \\
\text { access to }\end{array}$ & retaliation & All & $\begin{array}{l}\text { Don't } \\
\text { know }\end{array}$ \\
\hline Sukuro & $30.7(8)$ & $21.8(17)$ & $33.3(1)$ & $18.2(2)$ & $23.6(41)$ & 0 \\
Kimotorok & $7.7(2)$ & $25.6(20)$ & 0 & $9.1(1)$ & $11.5(20)$ & $1(3)$ \\
Lolkisale & $19.2(5)$ & $17.9(14)$ & $33.3(1)$ & $54.5(6)$ & $37.9(66)$ & 0 \\
Terrat & $42.3(11)$ & $34.9(27)$ & $33.3(1)$ & $18.2(2)$ & $27.0(47)$ & 0 \\
\hline
\end{tabular}

The question of law on the hunting and how the hunting and wildlife resources can be managed sustainably, interestingly received different views (Table 3.4b).

Table 3.4b: Respondents' answers in \% (n) for the question what should the wildlife law do to protect the wildlife around the area.

\begin{tabular}{llllll}
\cline { 2 - 5 } Villages & $\begin{array}{l}\text { Allow } \\
\text { hunting }\end{array}$ & $\begin{array}{c}\text { free } \\
\text { ban } \\
\text { hunting only }\end{array}$ & $\begin{array}{l}\text { local local and } \\
\text { trophy hunting }\end{array}$ & Don't know \\
\hline Sukuro & $19.4(7)$ & $29.7(43)$ & $17.5(20)$ & $33.3(1)$ \\
Kimotorok & $16.7(6)$ & $17.9(26)$ & $12.3(14)$ & 0 \\
Lolkisale & $33.3(12)$ & $32.4(47)$ & $28.1(32)$ & $66.7(2)$ \\
Terrat & $30.6(11)$ & $20.0(29)$ & $42.1(48)$ & 0 \\
\hline
\end{tabular}

Approximately $48.6 \%$ perceive that citizen hunting was critical to the wildlife survival and should be banned. Others $(38.2 \%)$ were suspicious of the legality of the 
hunting itself and suggested that all hunting be banned to rescue the wildlife from further declining. In contrast, $12 \%$ of the respondents had the feeling that the wildlife should be for all, rather than remaining a state resource and the law should allow free use of these resources without restrictions.

\section{Discussion}

\section{The poaching of wildlife in Masailand}

The poaching threats differed amongst species probably due to the species size and behaviour. For example the high proportion of impala being hunted may be attributed to its smaller body size and habit of thriving in disturbed habitats. The hunting and overall handling of impala by poachers may be easier than for other relatively big animals species (e.g. zebra and wildebeest), thus increasing its risks to poaching. Poachers hunt impala by using strong spot lights and sharp knives, arrows and some locals use pit traps (personal observation, 2005). The poaching of impala and Thomson's gazelle is primarily for local consumption and for selling meat at local black markets within the villages. At local restaurants in the Masailand it is not surprising to be served with the impala soup pretending to be of a goat. Unlike impala, zebra is hunted mainly for its skin (Park Warden personal communication, 2008). In this area the poaching of zebra and wildebeest is mostly by firearms and from vehicles. The meat and the zebra skins are supplied to the black market in big cities and sometimes exported to neighbouring countries, particularly Kenya. While the poaching incidences in the Masailand could be attribuTable to a lack of resource guards in the field, however, even in a heavily protected area like Serengeti National Park poaching remains a single major threat to the ecosystem (Arcese et al. 1995; Dublin et al, 1990). In the Serengeti National Park poaching is by the use of wire snares and night hunting with dogs and involves large groups of poachers hunting together (Arcese et al., 1995; Loibooki et al. 2002).

Poaching in the Masailand is driven by a number of external factors. Harsh economic conditions for the local people may incline them to hunt illegally as the immediate alternative source of food and cash income. This was voiced when the local people were asked for reasons why people would poach wildlife. In Serengeti National Park 
illegal hunting is conducted both for commercial and household food consumption (Kaltenborn et al., 2005; Loibooki et al., 2002).

As the Masai are increasingly being forced to cope with the current development change, young Masai get easily along with poachers and become involved in poaching of wildlife. Nelson (2000) points out that the Masai people assist poachers normally for monetary gains or for food when they come to hunt in the Masailand. A study done with local communities surrounding Serengeti National Park shows that about $34.3 \%$ of traders in the area rely on illegally acquired wild meat as their sole source of income (Barnett, 2000).

\section{Wildlife and the local people in Masailand}

The relationship between wildlife and local people has become important to conservation in many reserves throughout Africa. The apparent interaction between the wildlife and the agro-pastoral Masai give rise into conflicts. There are a multitude of problems reported by the Masai, ranging from loosing their cattle to lion predation, crop raiding by wild herbivores, to their cattle succumbing into malignant catarrh fever, a deadly disease believed to be transmitted from lambing wildebeest. There is no compensation for any loss when the local people encounter these problems. Further, unemployment is high and people currently benefit little from the wildlife resources. Due to insufficient wildlife incentives, these resources may be jeopardized in the hands of the Masai. Whether absolutely right or guilty by association, these conflicts have been a major source of wildlife deaths by Masai in this area. Carnivorebait poisoning was mentioned as a widespread practice to eliminate livestock predators around the area (Division Officer, personal communication, 2004). In this study, 11(4\%) respondents admitted that wildlife is killed in retaliation in their crop farms. Similar results have been reported in Kaltenborn et al. (2005) where about $38 \%$ of village communities admitted to killing animals that cause damage around Serengeti National Park. In Way Kambas National Park, Sumatra Indonesia, local people eliminate unwelcome animals (Nyhus et al., 2000). In Mozambique and Laikipia District, Kenya, local peasants and pastoralists perceive crop raiders and dangerous animals negatively (De Boer \& Baquete, 2002; Gadd, 2005).

The failure by the Masai to obtain sufficient wildlife-derived benefits has resulted in them having no affinity with the concept of conservation or the protection of wildlife 
against outside poachers. Beside them killing problem animals from their farms, I found that they also poach animals for subsistence and help outsiders in poaching wildlife. Similar finding has been reported by Nelson (2000) among the Masai communities in the Masailand.

\section{The effect of the double-barrelled question on the precision of poaching estimates}

The poaching estimates reported here may be over-estimates because of the nature of the question asked in the randomised response tool. Two or more respondents may have seen one poacher killing animals in the same area, hence reported many incidences while it is actually one event. Even if this is likely, however its impact on the precision of the estimates is minimal and less important due to the following;

First, I asked for only one incidence in the main question of the randomising method. While the chance for one respondent observing many different poaching events in different sites is large, respondents were asked to report on only one kill event. In the Masailand there are no wildlife guards who could oversee and protect these resources against poachers. As a consequence there may be repeated hunting by poachers who may take advantage of this freedom. Repeated poaching by same individuals has also been reported as a common phenomenon in the arrested poachers where they admitted to have gone hunting 4-times in one year in the Serengeti National Park (Hofer et al., 1996).

Second, in the randomised sensitive question respondents were not asked for how many animals they killed or seen killed by other poachers over the course of time. Due to the risks involved in poaching, poachers do not take one animal; rather they strive to maximize their hunt by killing as many animals as possible in one poaching occasion. This may happen because of the size of the animal hunted, easiness in handling (e.g. impala and Thomson's gazelle) and the resultant economic benefits from selling of the bushmeat (wildebeest and zebra). Thus it is likely that respondents gave one answer despite killing, or having seen killed many animals over the time period they were asked about. For example, in the study area, in one incidence about 24 skinless zebra carcases were found by rangers on a single patrol (Tarangire Warden's report, 2006). Similarly, among the Kurya tribe of Western Serengeti, 
illegal hunters are reported to kill up to or more than 80 animals in the Serengeti National Park in just one hunting trip (Hofer et al., 1996; Holmern et al., 2002; Loibooki et al., 2002).

Third, due to the widely distributed settlement patterns of the Masai, some poaching events around the area may go unnoticed by others who are located far from the scene. In this study I asked this question with further in-depth clarification on what I was looking for. I emphasised to the respondents that illegal hunting of animals could have been done by any means and for any cause. For example, the killing of wildlife in retaliation because of raiding crops in farms, hunting for home meat consumption as well as by assisting other people in the event of poaching the animal species in question.

Fourth, the sensitive nature of the poaching itself may have caused the respondents to respond for other poachers when they themselves had done the actual killing. While it is difficult to ascertain this, however Kaltenborn et al. (2005) found that within a well-known traditionally hunting tribe (the Kurya) adjacent to Serengeti National Park, when asked a direct question whether any member of the household is a hunter, $81 \%$ of respondents replied 'no'. Surprisingly, in the same study about $77 \%$ respondents said 'yes' when asked whether they knew of other hunters in the village. These mixed responses were attribuTable to the contentious nature of the poaching and its repercussions. For the Masai, the fear to disclosing poaching is probably justified from their long-standing trust to co-exist with the wildlife in many range areas. As most of their lives depend on livestock, anything that may deny them areas for grazing would be disastrous to their livelihoods. Collectively, these reasons nullify the bias that may have resulted from the question.

Nevertheless, the estimates reported here are lower compared to the poaching estimates reported in other studies elsewhere. For example, Hofer et al. (1996) estimated that about 160,000 migratory and resident animals are illegally harvested annually in the Serengeti National Park and associated areas. Similarly, approximately 40,000 wildebeest have been estimated to be poached annually from Serengeti National Park (Mduma et al., 1998). A more recent study by Loibooki et al. (2002) suggested that about 60,000 people are involved in illegal hunting of wildlife each 
year in the western side of Serengeti National Park. The current study is similar to these studies because poachers in both cases hunt resident and migratory animals wildebeest, zebra and impala. However, these studies differ with the current one in that they used spatial modelling techniques and arrest record with group meetings to derive the poaching estimates. None of these studies asked the hunting question to the local people directly as this study did.

\section{Implications for conservation}

The poaching levels are high and directly reduce the number of animals in the area. Both resident and migratory species are hunted by poachers. The migratory species, such as zebra and wildebeest, come from the adjacent Tarangire National Park where they enjoy full protection for about five months of the year. A reduction in number of these species will be reflected in the national park where these species are regarded as keystones (Lamprey, 1963). It is not known yet the long-term impact of this hunting on the population viability of the individual species in the area (see Chapter 6).

In the Masailand, poaching is associated with the level at which the local people are, or are not, satisfied with the current conservation benefits from the wildlife. It is also related to the financial and food insecurity amongst many people in the area. The shift in the mode of life from traditionally wandering to a more permanent settlement has further exacerbated the problem on the wildlife habitats. As more people settle down, they open more land for agricultural farms. This is already a threat to the important migratory corridors between the national parks and adjacent dispersal areas (Gamassa, 1995; Mwalyosi, 1992).

Any comprehensive strategy toward conservation in these areas should first address these irregularities on the ground. A comprehensive review of the current distribution of wildlife benefits within the Masai communities should be a priority. The government and responsible agencies running the hunting businesses around the area should strive to make the wildlife benefits more realistic to the Masai. This would involve strengthening the existing benefits (if any) and design for more wildlife projects targeted at increasing the individual and community benefits for the locals. The locals should have a sense of ownership into these projects. In this way they will protect these resources as theirs against outside poachers. Tourism activities in the 
form of wildlife ballooning may be useful and may be a suiTable candidate due to the plains nature of the area. Balloon tourism may be a complement for the existing hunting projects during seasons when hunting is closed and when almost all the animals of the Tarangire National Park are within these areas. Alongside increasing the wildlife benefits to the locals, the responsible wildlife authority should establish a patrol force in this area.

Education should also be an emphasis. Awareness for both age classes of the Masai community will improve their understanding and with benefits may improve their attitudes in those wildlife conflict-torn areas. Emphasis should be given on the environmental conservation education and how they can tape their livestock wealth into another form of capital which will minimize pressures on the environment and the wildlife habitats.

\section{Appendix 3.0 Case study animal species used in this thesis}

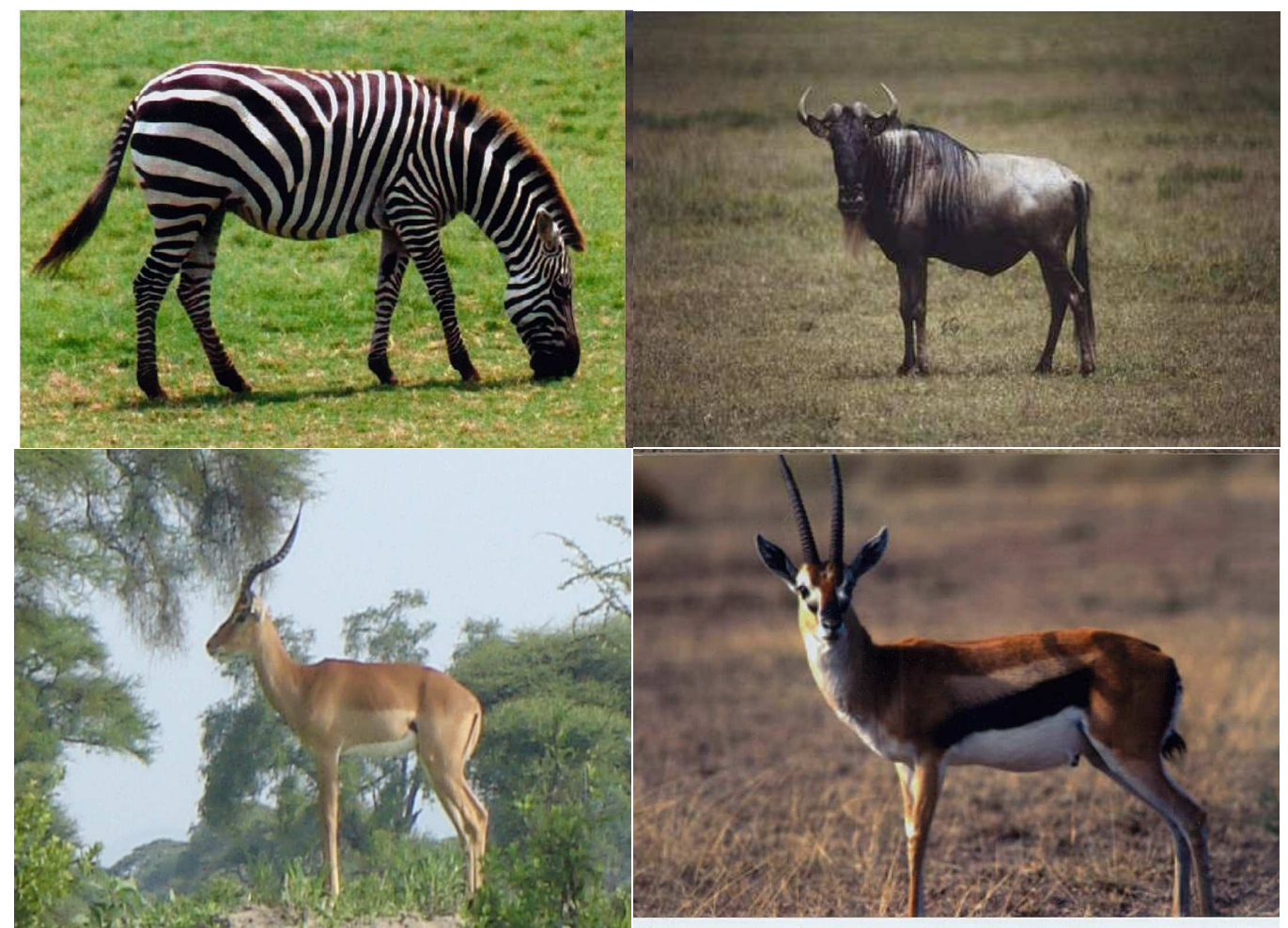




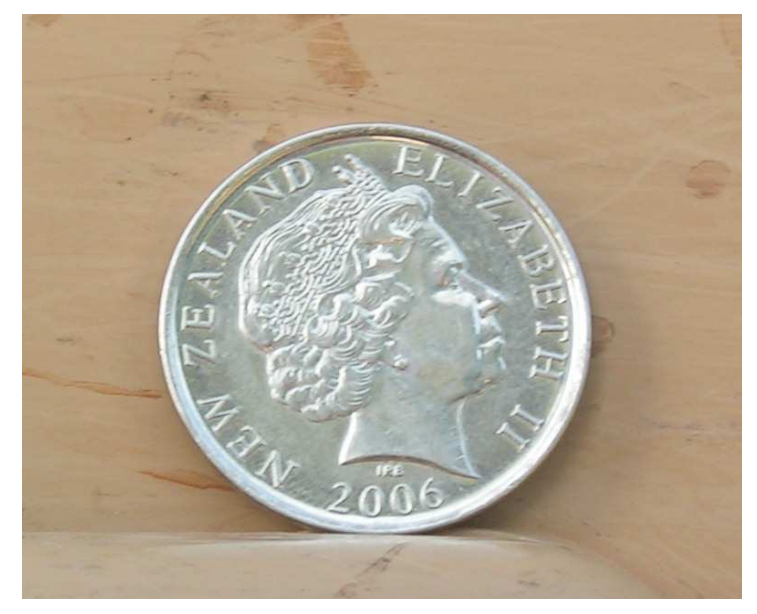

Photographs of the four wildlife species used as case study animals in this study. Top left-is Zebra, top-right is the Wildebeest, middle left-impala, middle right-Thomson's gazelle. The last (bottom) is a photo of a New Zealand 50cent coin. These photos were used along with the randomised response technique method in the study of poaching of these animals around the Tarangire National Park, northern Tanzania in 2008 (see chapter 3, of this thesis).

Appendix 3.1 Questionnaire survey used along with the Random Response technique (RRT) in the study of poaching of wildlife outside Tarangire-northern Tanzania-2008

1. What are the costs associated with having the wildlife living around your areas? Tick all that apply

a. wildlife compete for pastoral land with our livestock

b. wildlife destroy our crops in farms

c. wildlife kill our livestock e.g. lions

d. wildlife transmit disease to our livestock e.g. wildebeest

e.

2. What are the benefits do you get by living near these wildlife resources?

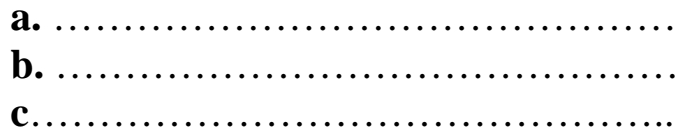

3. Is any member of your household family employed in any wildlife business sector such as parks, hunting company or tour company?
a. Yes
b. No

If yes how many people

4. Do you get any allowance/benefit from the government/company doing wildlife hunting business in these areas?
a. Yes
b. No 
If yes name those benefits

5. According to the time you have lived in this area, what can you say of the population trend of wildlife (seen in photos) in these areas? Tick one

(a) Animals have decreased much (b) Animals have increased much

(c) Neither decreased nor increased (d) I don't know

Give reason(s) for your answer above

6. Why do you think people would love to hunt/kill wildlife? Tick all that apply

a. want bush meat

b. need money from selling bush meat

c. wildlife is relatively easy to access to

d. want to get rid off problems caused by wildlife

e.

7. What economic activities you do that enable you earn your living? Tick all that apply

a. pastoral farming $\quad$ c. petty business

b. crop farming $\quad$ d. charcoal selling

8. On your opinion, what do you think on the law protecting wildlife in this area? Tick one which apply

a. The law should allow free access to wildlife

b. Local/community hunting should be prohibited by law

c. The law should stop any sort of hunting (local/tourist) in these areas

9. How did you find the applicability of this technique (RRT) in this study? Tick one which apply
a. easy to understand and apply
b. was difficult to understand
c. understandable even to illiterate

10. Which level of school did you attend? Circle one
a. standard seven ( class 1-7)
b. secondary/college education
c. No formal education 
11. For how long have you lived here (in this area)? Tick one
(a) Between 5-10 years
(b) Between 10-20 years
(c) Over 20 years

12. How old are you? Circle one
a. between $18-25$ years
b. between $25-45$ years
c. between $45-80$ years

\section{References}

Albers, H.J., \& Grinspoon, E. (1997). A comparison of the enforcement of access restrictions between Xishuangbanna Nature Reserve (China) and Khao Yai National Park (Thailand). Environmental Conservation, 24(04), 351-362.

Arcese, P.,Hando, J., \& Campbell, K.L.I. (1995). Historical and present day antipoaching efforts in Serengeti. In A. R. E. Sinclair, \& P. Arcese (Eds.), Serengeti. II. Dynamics, management, and conservation of an ecosystem (pp. 506-533). Chicago: University of Chicago Press. book chapter, pp. 506-533.

Barnett, R. (2000). Wildlife meat utilisation in the east and southern Africa region. In: links between biodiversity conservation, livelihoods and food security. The sustainable use of wild species for meat.(Eds S. Mainka and M.Trivedi. The IUCN Species survival commission, TRAFFIC East/Southern Africa, Nairobi, Kenya. .

Brashares, J.S.,Arcese, P.,Sam, M.K.,Coppolillo, P.B.,Sinclair, A.R.E., et al. (2004). Bushmeat hunting, wildlife declines, and fish supply in West Africa. Science, 306, 1180-1183.

Caro, T.M.,Pelkey, N.,Borner, M.,Campbell, K.L.I.,Woodworth, B.L., et al. (1998). Consequences of different forms of conservation for large mammals in Tanzania: preliminary analyses. African Journal of Ecology, 36(4), 303-320.

Chaloupka, M.Y. (1985). Application of the randomized response technique to marine park management: an assessment of permit compliance. Environmental Management, 9(5), 393-398.

De Boer, W.F., \& Baquete, D.S. (2002). Natural resource use, crop damage and attitudes of rural people in the vicinity of the Maputo elephant reserve, Mozambique. Environmental Conservation, 25(03), 208-218.

Dublin, H.T.,Sinclair, A.R.E.,Boutin, S.,Anderson, E.,Jago, M., et al. (1990). Does competition regulate ungulate populations? Further evidence from Serengeti, Tanzania. Oecologia, 82(2), 283-288.

Fischer, F., \& Linsenmair, K.E. (2001). Decreases in ungulate population densities. Examples from the Comoé National Park, Ivory Coast. Biological Conservation, 101(2), 131-135.

Fox, J., \& Tracy, P. (1986). Randomised response: a method for sensitive surveys. Beverly Hills. Sage Publication.

Gadd, M.E. (2005). Conservation outside of parks: attitudes of local people in Laikipia, Kenya. Environmental Conservation, 32(01), 50-63. 
Galaty, J.G. (1981). Introduction: nomadic pastoralists and social change processes and perspectives. Journal of Asian and African Studies, 16(1-2), 4-26.

Galaty, J.G. (1982). Being "Maasai"; being "people-of-cattle": ethnic shifters in East Africa. American Ethnologist, 9(1), 1-20.

Gamassa, D.G.M. (1995). Blockade of wildlife migration corridors by agricultural development in northern Tanzania. In: Integrating people and wildlife for a sustainable future (eds. Bissonette, J.A. \& Krausman, P.R.). The Wildlife Society, Bethesda, pp. 609-613.

Greenberg, B.G.,Abul-Ela, A.-L.A.,Simmons, W.R., \& Horvitz, D.G. (1969). The unrelated question randomized response model: theoretical framework. Journal of the American Statistical Association, 64(326), 520-539.

Gulliver, P.H. (1969). The conservative commitment in northern Tanzania: the Arusha and Masai. In P. H. Gulliver, ed. Tradition and Transition in East Africa. Berkeley and Los Angeles, University of California Press. Hailey, Lord. pp 223-42.

Hofer, H.,Campbell, K.L.I.,East, M., \& Huish, S.A. (1996). The impact of game meat hunting on target and non-target species in the Serengeti. In The exploitation of mammal populations: 117-146. Taylor, V. J. \& Dunstone, N. (Eds).London: Chapman and Hall.

Holmern, T.,Røskaft, E.,Mbaruka, J.,Mkama, S.Y., \& Muya, J. (2002). Uneconomical game cropping in a community-based conservation project outside the Serengeti national park, Tanzania. Oryx, 36(04), 364-372.

Houston, J., \& Tran, A. (2001). A survey of tax evasion using the randomized response technique. Advances in Taxation 13, 69-94.

Huntingford, G.W.B. (1955). The economic life of the Dorobo. Anthropos, 50, 602634.

Huntingford, G.W.D. (1953). The southern Nilo-Hamites. London, International African Institute, $150 \mathrm{P}$

I-Cheng, C.,Chow, L.P., \& Rowland, V.R. (1972). The randomized response technique as used in the Taiwan outcome of pregnancy study. Studies in Family Planning, 3(11), 265-269.

IUCN. (2007). IUCN red list of threatened species http://www.iucnredlist.org/info/stats.Gland, Switzerland.

Kahurananga, J. (1979). The vegetation of the Simanjiro plains, northern Tanzania. African Journal of Ecology, 17(2), 65-83.

Kahurananga, J. (1981). Population estimates, densities and biomass of large herbivores in Simanjiro plains, northern Tanzania. African Journal of Ecology, 19(3), 225-238.

Kaltenborn, B.,Nyahongo, J., \& Tingstad, K. (2005). The nature of hunting around the western corridor of Serengeti national park, Tanzania. European Journal of Wildlife Research, 51(4), 213-222.

Kideghesho, J.,Røskaft, E., \& Kaltenborn, B. (2007). Factors influencing conservation attitudes of local people in western Serengeti, Tanzania. Biodiversity and Conservation, 16(7), 2213-2230.

Kline, P.A., \& Schill, D.I. (1995). Use of random response to estimate angler noncompliance with fishing regulations. North American Journal of Fisheries Management 15, 721-731. 
Lamprey, H.F. (1963). Ecological separation of the large mammal species in the Tarangire game reserve, Tanganyika 1. African Journal of Ecology, 1(1), 6392.

Landsheer, J.,Van Der Heijden, P., \& Van Gils, G. (1999). Trust and understanding, two psychological aspects of randomized response. Quality and Quantity, 33(1), 1-12.

Lara, D.,Strickler, J.,Olavarrieta, C.D., \& Ellertson, C. (2004). Measuring induced abortion in Mexico: a comparison of four methodologies. Sociological Methods Research, 32(4), 529-558.

Leader-Williams, N.,Albon, S.D., \& Berry, P.S.M. (1990). Illegal exploitation of black rhinoceros and elephant populations: patterns of decline, law enforcement and patrol effort in Luangwa valley, Zambia. The Journal of Applied Ecology, 27(3), 1055-1087.

Lensvelt-Mulders, G.J.L.M.,Hox, J.J.,van der Heijden, P.G.M., \& Maas, C.J.M. (2005). Meta-analysis of randomized response research: thirty-five years of validation. Sociological Methods Research, 33(3), 319-348.

Loibooki, M.,Hofer, H.,Campbell, K.L.I., \& East, M.L. (2002). Bushmeat hunting by communities adjacent to the Serengeti national park, Tanzania: the importance of livestock ownership and alternative sources of protein and income. Environmental Conservation, 29(03), 391-398.

Mann, B.Q. (1995). Quantification of illicit fish harvesting in the Lake St Lucia game reserve, South Africa. Biological Conservation, 74(2), 107-113.

Mduma, S.A.R.,Hilborn, R., \& Sinclair, A.R.E. (1998). Limits to exploitation of Serengeti wildebeest and implications for its management. In: Dynamics of Tropical Communities, ed. D.M.Newbury, H.H.T. Prins \& N. Brown, pp. 243265. Oxford, UK: Blackwell Science.

Mwalyosi, R.B.B. (1991). Population growth, carrying capacity and sustainable development in south-west Masailand. Journal of Environmental Management, 33(2), 175-187.

Mwalyosi, R.B.B. (1992). Land-use changes and resource degradation in south-west Masailand, Tanzania. Environmental Conservation, 19(2), 145-152.

Nelson, F. (2000). Sustainable development and wildlife conservation in Tanzanian Maasailand. Environment, Development and Sustainability, 2(2), 107-117.

Newby, J.E. (1990). The slaughter of Sahelian wildlife by Arab royalty. Oryx, 24(1), 6-8.

Nyhus, P.J.,Tilson, R., \& Sumianto. (2000). Crop-raiding elephants and conservation implications at Way kambas national park, Sumatra, Indonesia. . Oryx, 34, 262-274.

Smith, D.R. (1989). The extent of fishing without a license in Colorado. M.S. Thesis. Colorado State University, Fort Collins, Colorado.

Smith, D.R.,Anderson, D.R., \& Smeltzer, J.F. (1989). Assessment of the violationsimulation method. Wildlife Society Bulletin, 17(2), 179-184.

Soeken, K.L., \& Damrosch, S.P. (1986). Randomized response technique: applications to research on rape. Psychology of Women Quarterly, 10(2), 119126.

Solomon, J.,Jacobson, S.,Wald, K., \& Gavin, M. (2007). Estimating illegal resource use at a Ugandan park with the randomized response technique. Human Dimensions of Wildlife, 12, 75-88. 
Stoner, C.,Caro, T.,Mduma, S.,Mlingwa, C.,Sabuni, G., et al. (2007). Changes in large herbivore populations across large areas of Tanzania. African Journal of Ecology, 45(2), 202-215.

Tezcan, S., \& Omran, A.R. (1981). Prevalence and reporting of induced abortion in Turkey: two survey techniques. Studies in Family Planning, 12(6/7), 262-271.

Thirgood, S.,Mosser, A.,Tham, S.,Hopcraft, G.,Mwangomo, E., et al. (2004). Can parks protect migratory ungulates? The case of the Serengeti wildebeest. Animal Conservation, 7(2), 113-120.

TNPR. (2006). Tarangire National Park protection report to Director of Tanzania National Parks. Arusha. Unpublished.

Umesh, U.N., \& Peterson, R.A. (1991). A critical evaluation of the randomized response method: applications, validation, and research agenda. Sociological Methods Research, 20(1), 104-138.

URT. (2002). Population and housing census of 2002 in Tanzania. http://www.tanzania.go.tz/census/reports.htm. Accessed on 3/8/2008.

Warner, S.L. (1965). Randomized response: a survey technique for eliminating evasive answer bias. Journal of the American Statistical Association, 60(309), 63-69.

Wright, L.V. (1980). Use of randomized response technique to estimate deer poaching. Wildlife Society Bulletin, 8(4), 342-344.

Wright, S.J.,Zeballos, H.,Dominguez, I.,Gallardo, M.M.,Moreno, M.C., et al. (2000). Poachers alter mammal abundance, seed dispersal, and seed predation in a Neotropical forest. Conservation Biology, 14(1), 227-239.

Yamagiwa, J. (2003). Bushmeat poaching and the conservation crisis in Kahuzi-Biega national park, Democratic Republic of Congo Journal of sustainable forestry, 16(3/4), $111-130$

Zwanenberg, R.M.v. (1976). Dorobo hunting and gathering: a way of life or a mode of production? African Economic History(2), 12-21. 


\section{LIFE HISTORY PARAMETERS OF ZEBRA, IMPALA, THOMSON'S GAZELLE AND WILDEBEEST IN THE TARANGIRE AREA, TANZANIA}

\section{Introduction}

Information about vital rates of exploited species is important to understanding how their populations might respond to human exploitation. For instance, how do birth and survival rates change in response to hunting? To better understand these tradeoffs, a record of species-specific demographic data is required. Unfortunately, species vital parameters such as the birth and death rates of juveniles and adults, the age and sex structures, gestation time, maximum rate of reproduction and life span require lengthy studies. Furthermore, these parameters may vary geographically which may suggest that for my study data from Tarangire area is necessary. This, however, is not possible and instead I have sought to collect these vital population parameters for zebra, wildebeest, impala and Thomson's gazelle from published literature for anywhere in sub-Saharan Africa.

\section{Life-history details of plains zebra}

Plains zebra are widely distributed throughout sub-Saharan Africa with the largest population still remaining in Kenya and Tanzania (Hack et al., 2004). The ecology and social behaviour of this species are well studied (Ginsberg \& Rubenstein, 1990; Klingel, 1969; Rubenstein, 1986). The conservation status of the species shows low decline, although this species is already extinct in some countries (e.g. Burundi, Lesotho and probably Angola) where originally it was plentiful (Hack et al., 2004). Habitat loss due to human activities and hunting (both illegal and legal) are the critical factors mooted for its continual decline in major parts of its range.

In Tanzania, life-history studies have been done for the Ngorongoro crater, Loliondo area, Serengeti National Parks in northern Tanzania (Klingel, 1975; Sinclair \& Norton-Griffiths, 1982; Smuts, 1976), and Rukwa area zebra in western Tanzania (Klingel, 1969). These studies show variations in the parameters recorded for different zebra populations and even for the same population recorded at different years. For example, for the Ngorongoro zebra alone, the proportion of female zebra giving birth to foals increased from $38 \%$ in 1987 to $46 \%$ in 1988, while the male: 
female sex ratio of foals dropped from 1.3 to 1.1 in the same years (Hack et al., 2004). Even for the same population, female fecundity rates and yearling sex ratio are available only for one year (Table 4.0). The population of plains zebra in Serengeti national Park appears to be regulated by predators (Grange et al., 2004; Sinclair \& Norton-Griffiths, 1982). This suggests that the vital rates of this population would probably differ from a population that is not constrained by predators, e.g. the Simanjiro plains zebra where predators (lions and hyenas) have been heavily decimated by pastoralists. Further, the Simanjiro plains zebra faces pressure from resource competitors, livestock, suggesting that its population would still behave differently from its counterpart.

Plains zebras reproduce polygynously whereby a single stallion may collect up to ten mares in a harem (Klingel, 1969; Rubenstein, 1986). Variation in local climate conditions (i.e. annual rainfall) greatly influences on the patterns of recruitment rates, foal survival, adult sex ratio and population density (Pettorelli et al., 2005; Rubenstein, 1986; Toïgo \& Gaillard, 2003). In the Simanjiro plains of northern Tanzania, plains zebras were observed giving birth to fewer foals during seasons of droughts (personal Observation, 2005). This confirms reports of restricted female reproduction when resources are scarce (Albon et al., 1983; Festa-Bianchet \& Jorgenson, 1998). In the Serengeti National park, the recruitment rates of most herbivores are rainfall-mediated (Pascual et al., 1997), and thus of the foal mortality and fecundity rates due to resource availability (Gaillard et al., 2000). Thus, the study in western Tanzania, however, gives insights for what could probably be for the zebra population in northern Tanzania, as western Tanzania receives relatively high rainfalls than the northern part.

Plains zebra have been studied in Samburu National Park and Athi-Kapiti plains in Kenya (Ohsawa, 1982; Petersen \& Casebeer, 1972; Rubenstein, 1989). These studies examined group composition, recruitment and mortality rates and sex ratios amongst adults (Table 4.0). Although these studies give a comprehensive life Table for the plains zebra, the age distribution of that population is unlikely to be representative of all zebra populations. Currently, however, these studies are the only available sources of such data and indeed have been used in stochastic Leslie matrix population projection models, e.g. Georgiadis et al. (2003). 
From the southern Africa regions plains zebra have been studied in Nyika National Park, Malawi (Munthali \& Banda, 1992), in Etosha National Park, Namibia (Gasaway et al., 1996; Klingel, 1969) and in Kruger National Park, South Africa (Gasaway et al., 1996; Klingel, 1969; Smuts, 1976). The study at Nyika is less detailed and does not depict important vital rates apart from showing the adult sex ratio and recruitment of foals. As for the Etosha's population, the study is confounded by the same constraints although it records group composition and juvenile recruitment rates.

Table 4.0: Demographic variables from published literature for plain zebra Populations throughout their eastern and southern Africa range

\begin{tabular}{|c|c|c|c|c|c|c|c|}
\hline $\begin{array}{l}\text { Studied zebra } \\
\text { populations }\end{array}$ & $\begin{array}{l}\text { Annua } \\
\text { mortal } \\
\text { rate } \\
\text { Foals }\end{array}$ & & $\begin{array}{l}\text { Age, } 1 \text { st } \\
\text { reproduction }\end{array}$ & $\begin{array}{l}\text { Recruit } \\
\text { rates } \\
\text { Foals } \\
(=1 \mathrm{yr})\end{array}$ & 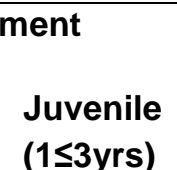 & $\begin{array}{l}\text { Adult } \\
\text { sex ratio } \\
\text { M:F }\end{array}$ & $\begin{array}{l}\text { Source } \\
\text { s }\end{array}$ \\
\hline Ngorongoro Crater, & & $0.03-$ & & & & $0.69-$ & \\
\hline $\begin{array}{l}\text { Tanzania } \\
\text { Ngorongoro Crater, }\end{array}$ & 0.19 & 0.09 & & & 0.2 & 0.88 & 4,5 \\
\hline & 0.19 & 0.07 & $3.40-4.40$ & 0.19 & 0.19 & & 6 \\
\hline Tanzania & & & & 0.11 & & 0.56 & 12 \\
\hline Serengeti NP, Tanzania & 0.3 & 0.11 & & 0.39 & 0.85 & & 4,7 \\
\hline $\begin{array}{l}\text { Samburu N.R., Kenya } \\
\text { Athi-Kapiti Plains, }\end{array}$ & 0.33 & & & 0.2 & 0.12 & 0.62 & 1,2 \\
\hline $\begin{array}{l}\text { Kenya } \\
\text { Etosha NP, Namibia }\end{array}$ & 0.38 & 0.17 & & 0.26 & $\begin{array}{l}0.28 \\
0.18\end{array}$ & & $\begin{array}{l}3 \\
4,11\end{array}$ \\
\hline Nyika NP, Malawi & & & & 0.1 & & 1 & 8 \\
\hline $\begin{array}{l}\text { Kruger NP, South } \\
\text { Africa }\end{array}$ & 0.47 & $\begin{array}{l}0.03- \\
0.13\end{array}$ & & 0.12 & 0.06 & 0.75 & 9.10 \\
\hline
\end{tabular}

References: 1- Rubenstein (1989); 2- Ohsawa (1982); 3- Petersen and Casebeer (1972);

4-Klingel (1969); 5-Klingel (1975); 6- M. Hack

http://www.eeb.princeton.edu/FACULTY/Rubenstein/pdf_dir/2002_Hack\%20etal_IUNC_Moehlman_e d.pdf (accessed May, 2008); 7-Grange et al. (2004); 8-Munthali and Banda (1992); 9- Smuts (1976a); 10-Smuts (1976b); 11-Gasaway, et al. (1996); 12-Skoog in Smuts (1976a).

From the southern region, the only detailed and comprehensive study was done at Kruger NP (above). Authors have shown variables that are important at least for species population survival (Table 4.0). When examined, the southern plains zebra population differ markedly with the eastern Africa population. For example, plains zebra on Athi-Kapiti plains in Kenya recruit foals at $26 \%$ yearly while at Kruger NP, only $12 \%$ foals are recruited. Similarly, $28 \%$ of juveniles are recruited into the adult population in Kenya and only $6 \%$ juveniles are recruited at Kruger NP. This 
difference may be explained by differences in climatic conditions and predators between the sites. It is not known whether this variation also holds for the Tarangire plains zebra. Table 4.1 shows the survival and fecundity rates of zebra population in the Serengeti National Park.

Table 4.1: Survival and fecundity rates of zebra population in Serengeti National Park.

\begin{tabular}{lcc}
\hline Age & Fecundity & Survival \\
\hline First year & 0 & 0.389 \\
Yearling & 0 & 0.847 \\
2-year olds & 0 & 0.979 \\
3-5 year olds & 0.686 & 0.954 \\
Mature female & 0.883 & 0.875 \\
Senescent female & 0.883 & 0.768 \\
\hline
\end{tabular}

Data taken from Grange et al. 2004

\section{Life history parameters of Impala}

Impala are unique browsers in the savannahs of Africa occurring widely through the wooded grassland and open woodland zones of western, central and southern Africa. Impala form large groups composed of many females with a single territorial male and they reproduce polygynously. The southern impala have a 3-week rut at the end of the rainy season (Jarman \& Jarman, 1973). The East African impala, however, have a more extended breeding period with most females conceiving late in the rainy season. A full description of the social behaviour and reproduction of impala is given by (Jarman, 1974; Jarman \& Jarman, 1973). Information about life history variables of impala is scant and only a few studies have attempted to study impala in natural range (Dasmann \& Mossman, 1962; Jarman \& Jarman, 1973). Females can conceive at 1.5 years age and males mature by the age of 4 (Jarman, 1979). The gestation period, birth rate, life span and age at first reproduction were sourced from these studies (above), as is shown in Table 4.2. 
Table 4.2: Life history parameters of the eastern impala population. Data sourced from studies above.

\begin{tabular}{ll} 
Parameter & Range/values \\
\hline Age at 1st reproduction males & 5 \\
Age at 1st reproduction females & 2 \\
Inter-birth interval & 1.1 \\
Mating system & polygynous \\
Number of young & 1 \\
Sex ratio of young & $50 \%$ \\
Sex ratio of adult & $60 \%$ \\
Annual mortality- juveniles(F\&M) & $40 \%$ (both) \\
Yearling mortality(M/F) & $20 \% \mathrm{M}$ and $10 \% \mathrm{~F}$ \\
Annual mortality adult males & $0-25-66-100 \%$ \\
& $($ various ages) \\
Annual mortality adult females & $3-5-30-20 \%$ \\
Maximum age & $($ various ages) \\
Fecundity rates & 13 \\
& $70-90 \% 1^{\text {st }}$ and later age \\
\hline
\end{tabular}

\section{Life history parameters of Thomson's gazelle}

The range of a Thomson's gazelle is quite small, consisting only of areas in Tanzania, Kenya, and southern Sudan. They live in areas of mostly short grasslands of the open savannah (Hosking \& Withers, 1996). However they will move to more wooded areas when the supply of grass becomes low during the dry season (Estes, 1967). The behaviour and ecology of Thomson's gazelle have been described in many studies (Baptist \& Fink, 1992; Estes, 1967; Estes et al., 2006; Robinette \& Archer, 1971). However, life history information about this species is poorly documented. The available few data indicate that this species become sexually mature at the age of one and two years for the female and male respectively (Robinette \& Archer, 1971). As in other harem forming ungulates, males form territories containing up to sixty females in a discrete and lose group. This group may sometimes be difficult to serve in the presence of other competing (satellite) males. However, as this species range is small, any available information may be suiTable or representative of all other populations. The available parameters were sourced from a range of studies done in Tanzania and Kenya (Table 4.3). 
Table 4.3: Life-history parameters of Thomson's gazelle

\begin{tabular}{lll} 
Parameter & Range/values & $\begin{array}{l}\text { Survival } \\
\text { rates }\end{array}$ \\
\hline Age at 1st reproduction males & 2 years & $\mathrm{b}$ \\
Age at 1st reproduction females & $\begin{array}{l}\text { 1 year } \\
0.5 \text { year } \\
\text { polygynous }\end{array}$ & $\mathrm{a}, \mathrm{b}$ \\
Inter-birth interval & 1 & \\
Mating system & 0.5 & $\mathrm{~b}$ \\
Number of young & $0.45-0.70$ & $\mathrm{~b}$ \\
Sex ratio of young & unknown & \\
Annual mortality- juveniles & 0.22 & $\mathrm{C}$ \\
Yearling mortality & 0.22 & $\mathrm{C}$ \\
Annual mortality adult males & $10.5-12$ years & $\mathrm{a}, \mathrm{b}$ \\
Annual mortality adult females & & \\
Maximum age & $0.27-0.28$ & $\mathrm{~d}$ \\
Survival rates & $0.098-0.12$ & $\mathrm{~d}$ \\
Calves & & \\
Yearlings & &
\end{tabular}

Data source: a. Hosking \& Withers (1996); b. Robinette \& Archer (1971); c. Baptist \& Fink (1992); d. Estes et al. (2006).

\section{Life history parameters of Wildebeest}

Wildebeest is perhaps the most widely dispersed species in the savannahs of east Africa. It is, however, declining in most parts of its range due to anthropogenic disturbance (Estes et al., 2006). The wildebeest population has been studied extensively across large part of its range (Gasaway et al., 1996; Kahurananga, 1981; Munthali \& Banda, 1992; Sinclair \& Norton-Griffiths, 1982). Wildebeest reproduce polygynously and exhibit a synchronous breeding pattern where pregnant females drop their calves almost during the same period (Sinclair, 1977). The females give birth to only one calf from the age of one and a half to two years as opposed to males who attain sexual maturity at the age of 3-4 (Talbot \& Talbot, 1963). The maximum age recorded for the wildebeest is 20 years (Hosking \& Withers, 1996; Talbot \& Talbot, 1963). The patterns of reproduction and of calf mortality appear to be influenced by the range nutrition. Under drought conditions most females tend to breed at a later age or not at all, this has been demonstrated in other wild ungulate species e.g. deer (Gaillard et al., 2000; Talbot \& Talbot, 1963). In the Serengeti wildebeest most (75\%) cases of mortality was due to undernutrition (Mduma et al., 1999). The eastern Masailand wildebeest (Athi-Kapiti plains, Kenya and Simanjiro plains, Tanzania) has poor nutrition due to competition from grazing livestock. As a 
consequence, the wildebeest populations in these areas have lower calf survival rates than in the Serengeti population (Talbot \& Talbot, $1963 \&$ references there in).

The male to female ratio both at birth and adulthood differs among the Serengeti and Tarangire wildebeest populations. In the Serengeti, males constitutes about $54 \%$ of all born calves and about 52\% at adulthood whereas in the Tarangire wildebeest about $33 \%$ of adults are male (Talbot \& Talbot, 1963). The differential birth and mortality rates between these populations have been explained by the different plant phenology, human activities (e.g. overgrazing) and other adverse conditions in these areas causing a disproportionate prenatal and postnatal mortality of males. High loss of males especially under adverse and overstocked range conditions has been discussed in relation to herds of mountain goats, elk, caribou and mule deer (Cowan, 1950 cited in Talbot \& Talbot, 1963). Similarly, harsh environmental conditions has also been shown to have a bigger impact on most observed lower survival of males than females in most ungulates species (Toïgo \& Gaillard, 2003).

The vital rates for the wildebeest population has been calculated from the Serengeti ecosystem (Grange et al., 2004; Talbot \& Talbot, 1963) and the age structure is shown in Table 4.4. Based on the local climate and plant phenology which are about the same in Tarangire and Serengeti national parks (Lamprey, 1964), these parameters will be used as surrogates for the Tarangire wildebeest models.

Table 4.4: Life-history parameters of wildebeest from Eastern Africa

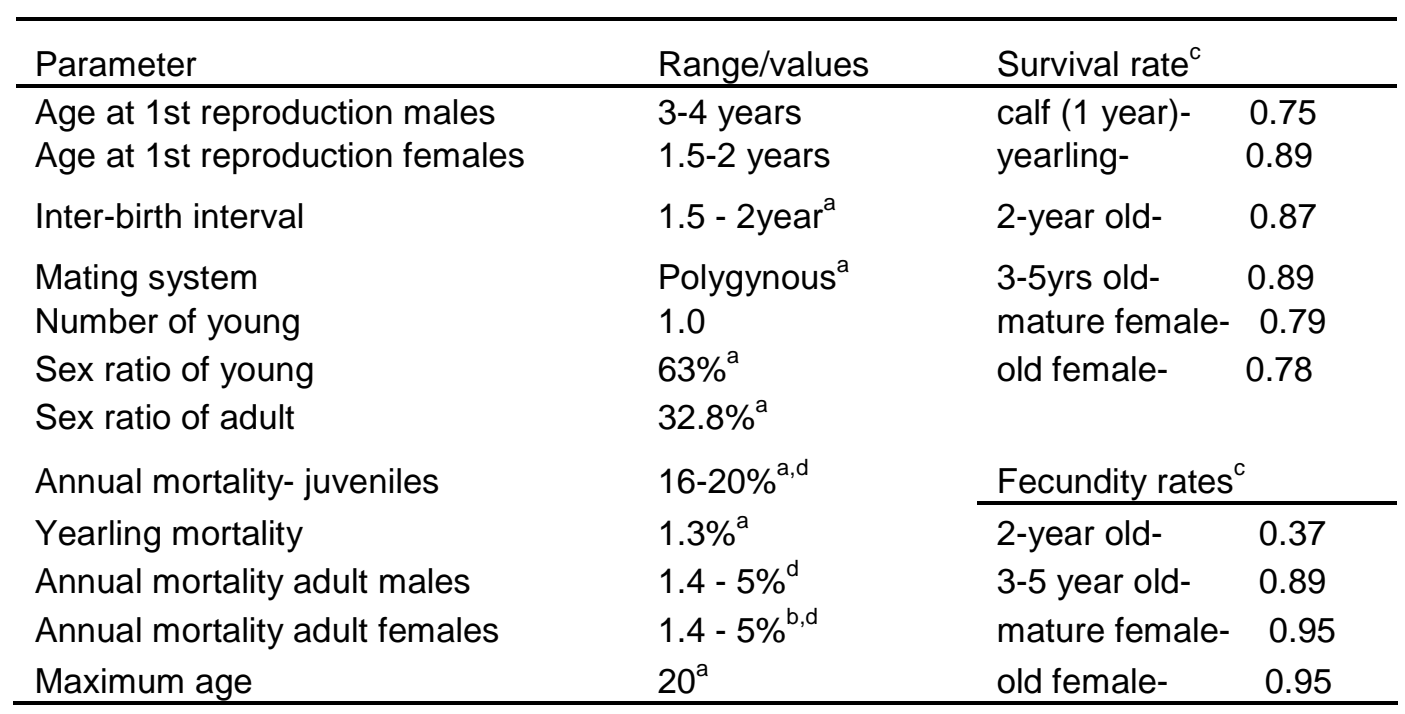


Data sources: a- Talbot \& Talbot (1963), b- Mduma et al.(1999), c- Grange et al.(2004), d- Baptist \& Fink (1992)

\section{Size and life-history (Allometric relationships)}

In the past decades, allometric laws have been used to predict important life history parameters of mammals. It has been argued that size scales main life history variables of mammals and that it can be used to determine gestation time, birth rate, age at first reproduction, intrinsic rate of natural increase, and life span (Western, 1979). A growing body of literature shows that size has been used to predict population parameters such as life span (Sacher in Western, 1979), reproductive rate (Fenchel, 1974) and growth rate (Millar, 1977). These studies are intuitive and provide baseline information on these parameters; however they provide little information useful for population projection studies. Predicting population parameters using body size relative to physiological and biochemical processes of mammals gives generalised results about the species. Such estimation does not take into account the role of environmental variables and their influences on population parameters. Thus, by themselves, they are not likely to be useful when attempting population dynamics studies. Additionally, this method can not be used to predict the vital rates for a specific species population such as the mortality and survival rates nor scales these parameters into age-class structure. Nevertheless, some parameters though general, are useful for a species population survival at any one point in time, namely birth rate, gestation time and life span (Table 4.5).

Table 4.5: Life history parameters of target animals from around the species range

\begin{tabular}{llllll}
\hline Species & $\begin{array}{l}\text { Birth rate } \\
\text { \% per year }\end{array}$ & $\begin{array}{l}\text { Gestation } \\
\text { (days) }\end{array}$ & $\begin{array}{l}\text { age } \mathbf{1}^{\text {st }} \\
\text { Reproduction }\end{array}$ & $\begin{array}{l}\text { life span } \\
\text { (years) }\end{array}$ & references \\
\hline T.gazelle & 59 & 191 & 1.5 & 11 & $1,2,3$ \\
Impala & 36 & $191-200$ & $1.5-1.9$ & 13 & $1,4,5$ \\
Zebra & 22 & 363 & 3 & 26 & $1,5,6,7$ \\
Wildebeest & 25 & 234 & 2.5 & $17-20$ & 1 \\
\hline
\end{tabular}

References: 1-7 respectively, (Coe et al., 1976; Dasmann \& Mossman, 1962; Klingel, 1969; Leuthold, 1972; Petersen \& Casebeer, 1972; Robinette \& Archer, 1971; Spinage, 1972)

Species population profiles from time-specific life-tables have been importantly recognized and used in the field of biology. However, being mostly derived from serendipitously collected skulls and age-specific census sightings, e.g. Spinage (1972) 
(Spinage, 1972) they can hardly give the actual age structure of population. The chance-encounter collection method relied on results into small samples as most of the skulls are missed out due to dissociation or ineffective search efforts. Moreover, constructing life-tables is difficult due to obligatory assumptions which have to be fulfilled (Gaillard et al., 1998). The life-table technique assumes a stationary age distribution and equal probability of sampling for all individuals. Unless these restrictive assumptions are met, survival estimates are unreliable (Caughley in Gaillard et al, 1998). Additionally, in real field environments, these strict assumptions are unlikely to be met in any population of wild mammals (Menkens \& Boyce, 1993). Even if the assumptions were met, life-table methods do not generally provide any measure of variability in survival rates (Spinage, 1972). Nevertheless, crude information from life-tables can prove useful in the absence of any surrogate data.

\section{Chapter summary}

In this chapter I collated data on the life history parameters for zebra, impala, Thomson's gazelle and wildebeest from across their ranges in sub-Saharan Africa. These data will be used as surrogates for the Tarangire populations in the population viability models (see Chapter 6).

\section{References:}

Albon, S.D.,Mitchell, B., \& Staines, B.W. (1983). Fertility and body weight in female red deer: a density-dependent relationship Animal Ecology, 52(3), 969-980

Baptist, R., \& Fink, H. (1992). Herd dynamics modelling applied to a venison ranch in Kenya. In World animal review, ( 73), (available at http://www.fao.org/ag/AGa/AGAP/FRG/FEEDback/War/u8750b/u8750b04.ht m.)

Coe, M.J.,Cumming, D.H., \& Phillipson, J. (1976). Biomass and production of large African herbivores in relation to rainfall and primary production Oecologia, 22(4), 341-354

Dasmann, R.F., \& Mossman, A.S. (1962). Population studies of impala in southern Rhodesia. Journal of Mammalogy, 43(3), 375-395.

Estes, R.D. (1967). The Comparative behavior of Grant's and Thomson's Gazelles. Journal of Mammalogy, 48(2), 189-209.

Estes, R.D.,Atwood, J.L., \& Estes, A.B. (2006). Downward trends in Ngorongoro Crater ungulate populations 1986-2005: conservation concerns and the need for ecological research. Biological Conservation, 131(1), 106-120.

Fenchel, T. (1974). Intrinsic rate of natural increase: the relationship with body size. Oecologia, 14( 4), 317-326 
Festa-Bianchet, M., \& Jorgenson, J.T. (1998). Selfish mothers: reproductive expenditure and resource availability in bighorn ewes. Behavioural Ecology, 9(2), 144-150.

Gaillard, J.-M.,Festa-Bianchet, M.,Yoccoz, N.G.,Loison, A., \& Toigo, C. (2000). Temporal variation in fitness components and population dynamics of large herbivores. Annual Review of Ecology and Systematics, 31(1), 367-393.

Gaillard, J.M.,Festa-Bianchet, M., \& Yoccoz, N.G. (1998). Population dynamics of large herbivores: variable recruitment with constant adult survival. Tree, 13(2), 58-63.

Gasaway, W.C.,Gasaway, K.T., \& Berry, H.H. (1996). Persistent low densities of plains ungulates in Etosha National Park, Namibia: testing the food-regulating hypothesis Canadian Journal of Zoology, 74(8), 1556-1572.

Ginsberg, J., \& Rubenstein, D. (1990). Sperm competition and variation in zebra mating behavior. Behavioral Ecology and Sociobiology, 26(6), 427-434.

Grange, S.,Duncan, P.,Gaillard, J.-M.,Sinclair, A.R.E.,Gogan, P.J.P., et al. (2004). What limits the Serengeti zebra population? Oecologia, 140(3), 523-532.

Hack, M.A.,Rod, E., \& Rubenstein, D.I. (2004). Status and action plan for the plains zebra. (Equus burchellii). www.eeb.princeton.edu/faculty/Rubenstein/pdf_dir. accessed on 1 sept.2008, 43-59.

Hosking, D., \& Withers, M.B. (1996). Safari guides: larger mammals of East Africa. Harper Collins Publishers, London.

Jarman, M.V. (1979). Impala social behaviour : territory, hierarchy, mating, and the use of space. Berlin; Hamburg: Parey.

Jarman, P.J. (1974). The social organisation of antelope in relation to their ecology. Behaviour, 48(3/4), 215-267.

Jarman, P.J., \& Jarman, M.V. (1973). Social behaviour, population structure and reproductive potential in impala. African Journal of Ecology, 11(3-4), 329338.

Kahurananga, J. (1981). Population estimates, densities and biomass of large herbivores in Simanjiro plains, northern Tanzania. African Journal of Ecology, 19(3), 225-238.

Klingel, H. (1969). The social organization and population ecology of the plains zebra (Equus quagga). Zoologica Africana, 4, 249-263.

Klingel, H. (1975). Social organization and reproduction in equids. Reproduction and fertility. Supplement, 23, 7-11.

Lamprey, H.F. (1964). Estimation of the large mammal densities, biomass and energy exchange in the Tarangire game reserve and the Masai steppe in Tanganyika. African Journal of Ecology, 2(1), 1-46.

Leuthold, W. (1972). Gestation period in Thomson's gazelle. African Journal of Ecology, 10(4), 309-310.

Mduma, S.A.R.,Sinclair, A.R.E., \& Hilborn, R. (1999). Food regulates the Serengeti wildebeest: a 40-year record. Journal of Animal Ecology, 68(6), 1101-1122.

Menkens, G.E., \& Boyce, M.S. (1993). Comments on the use of time-specific and cohort life Tables. Ecology, 74, 2164-2168.

Millar, J.S. (1977). Adaptive features of mammalian reproduction Evolution, 31(2), 370-386

Munthali, S.M., \& Banda, H.M. (1992). Distribution and abundance of the common ungulates of Nyika National Park, Malawi. African Journal of Ecology, 30(3), 203-212. 
Ohsawa, H. (1982). Transfer of group members in plain zebra (Equus burchelli) in relation to social organization. African study Monographs, 2, 53-71

Pascual, M.A.,Kareiva, P., \& Hilborn, R. (1997). The influence of model structure on conclusions about the viability and harvesting of Serengeti wildebeest. Conservation Biology, 11(4), 966-976.

Petersen, J.C.B., \& Casebeer, R.L. (1972). Wildlife management in Kenya. Distribution, population status and group composition of wildebeest (Connochaetes taurinus Burchell) and zebra (Equus burchelli Gray) on the Athi-Kapiti plains, Kenya Project KEN/71/526, Nairobi, Kenya.

Pettorelli, N.,Gaillard, J.M.,Yoccoz, G.N.,Duncan, P.,Maillard, D., et al. (2005). The response of fawn survival to changes in habitat quality varies according to cohort quality and spatial scale. Journal of Animal Ecology, 74(5), 972-981.

Robinette, W.L., \& Archer, A.L. (1971). Notes on ageing criteria and reproduction of Thomson's gazelle. African Journal of Ecology, 9(1), 83-98.

Rubenstein, D.I. (1986). Ecology and sociality in horses and zebras. Pp. 282-302. In: Ecological Aspects of Social Evolution. D. I. Rubenstein \& R. W. Wrangham, (eds.). Princeton University Press, Princeton, NJ.

Rubenstein, D.I. (1989). Life history and social organization in arid adapted ungulates. Journal of arid environments 17(2), 145-156

Sinclair, A.R.E. (1977). Lunar cycle and timing of mating season in Serengeti wildebeest. Nature, 267, 832-833.

Sinclair, A.R.E., \& Norton-Griffiths, M. (1982). Does competition or facilitation regulate migrant ungulate populations in the Serengeti? A test of hypotheses. Oecologia, 53(3), 364-369.

Smuts, G.L. (1976). Population characteristics of Burchell's zebra (Equus burchelli antiquorum, H. Smith, 1841) in the Kruger National Park

South African Journal of Wildlife Research, 6, 99-112.

Spinage, C.A. (1972). African ungulate life Tables. Ecology, 53(4), 645-652.

Talbot, L.M., \& Talbot, M.H. (1963). The wildebeest in western Masailand, East Africa. Wildlife Monographs(12), 3-88.

Toïgo, C., \& Gaillard, J.M. (2003). Causes of sex-biased adult survival in ungulates: sexual size dimorphism, mating tactic or environment harshness? Oikos, 101(2), 376-384.

Western, D. (1979). Size, life history and ecology in mammals. African Journal of Ecology, 17(4), 185-204. 


\section{DENSITY OF WILDLIFE POPULATIONS OUTSIDE TARANGIRE NATIONAL PARK, TANZANIA}

\section{Introduction}

Understanding the trend of a wildlife population is at the forefront of its conservation. In ecosystems constrained by complex and both deterministic and stochastic influences a regular monitoring of population changes is required. Reliable and accurate estimates of population size are crucial for assessing impacts of management practices on the species population dynamics, amongst others.

The Masailand, a Game controlled area outside of Tarangire National Park harbours substantial resident and migrant species of game animals. This area is also home to a human population and its associated activities. As a consequence of these increasing human settlements, the wildlife habitats have continued to shrink. Hunting of the animals and destruction of important migratory corridors is high and requires management intervention. It has been pointed out that many similar areas over the country are experiencing declines of wildlife (Caro et al., 1998b). Thus any attempt to halt these declines should first address the status of species in the area. In this study, I determine the abundance of game animals; zebra (Equus burchellii Matschie), impala (Aepyceros melampus Matschie), wildebeest (Connochaetes taurinus Thomas) and Thomson's gazelle (Gazella thomsonii Günther). These animals form a significant proportion of wildlife biomass in the area (Kahurananga, 1981) and are the most hunted of the game species.

Survey of the Masailand ungulate population was pioneered by Lamprey (1964) and later studied by Kahurananga (1981) and has subsequently been done by the Tanzania Wildlife Conservation Monitoring (TWCM) unit. Much of the previous work concentrated on the Simanjiro plains (part of Masailand) because of the plains' importance as the wet season refuge and calving ground for the Tarangire park immigrants. These surveys mostly use aerial census (Systematic Reconnaissance Flight) methods which have been amply developed and refined (Norton-Griffiths, 1978) and are suitable for surveying ungulates throughout the East African savannah. Aerial survey is advantageous because it covers a wide area in a relatively short time. 
The downside of aerial census however, is an inability to see and count small, medium size, or cryptic antelopes and it is limited for species living under vegetation (Dunbar, 1990; Komers, 1996). For example, there are no records of Thomson's gazelle population data in the recent aerial counts done by TWCM in the area, a probable consequence of this method. Furthermore, as aerial counting can miss some objects on the grounds there is a potential for imprecisely estimating the population density of animals. Therefore under such circumstances, ground survey methods are needed for species that can not be counted from the air and for potentially increasing the accuracy of aerial data.

Ground survey methods such as strip transect and road counts have been attempted for wildlife population estimates elsewhere (Caro, 1999a; Estes et al., 2006; Gasaway et al., 1996; Munthali \& Banda, 1992). They provide indices of abundance and spatial distribution of wildlife in reserves hence form a basis for evidence-based management. However, because these methods use strips of fixed width in which animals are exclusively counted, the potential for estimating the overall population size imprecisely is high. Consequently the data generated are limited in the use, especially when attempting population projection studies which demand more accurate data. In this paper I used the distance sampling method (Buckland et al., 2001) to estimate the number of each species in Simanjiro plains, Tanzania, a study area of $570 \mathrm{~km}^{2}$.

\section{Methods}

\section{Survey design}

Distance sampling (Buckland et al., 2001) requires that critical assumptions be met in the field for accurate density estimation. These assumptions include: 1. all animals on the line be detected and counted (i.e. detection function for a line equals 1); 2. no animal movement in response to observers prior to detection; and 3. all measurements are recorded accurately. Data collection was carried out during January and February 2008, at start of the wet season when visibility was good.

The design of the survey and subsequent layout of transects took into account the distribution of human activities such as farms, villages, and hunting camps present in the area. This design also ensured that a maximum area needed to be surveyed was 
adequately covered. I used a map of the area to identify and randomly distribute a total of 21 transect lines in the area. The transects were $10 \mathrm{~km}$ long except for six transects which measured from $2-9 \mathrm{~km}$ due to limited accessibility to some of the selected transects by vehicle (Fig. 2). Transects were at approximate equal intervals to avoid overlapping and double counting of the animals except when limited by physical barriers and human activities. The total transect length was $196.2 \mathrm{~km}$ long. Starting points for the survey transects were randomly chosen and its GPS coordinate point was recorded.

A four-wheel drive vehicle was driven along each transect with one recorder seated inside and an observer standing above the vehicle. When target animals were seen, the vehicle was stopped and I recorded the number of animals encountered (herd size), the distance to the animals (sighting distance, $\left.\chi_{\mathrm{i}}\right)$ and the sighting angle $\left(\theta_{\mathrm{i}}\right)$ which was the angle subtended between the line of travel and the animal or herd (Buckland et al., 2001). I used a GPS (Garmin 60) to mark the coordinates of the location of sighting of the animals and kept tracks of all the points from the start to the end of each transect. A Bushnell range finder accurate to a maximum distance of $1500 \mathrm{~m}$ was used to measure the sighting distance and a hand held compass bearing for angle measurement. The range finder had built-in high powered binoculars which facilitated counting of distant animals. Total transect length was taken from a vehicle odometer after every $10 \mathrm{~km}$ drive on transects unless otherwise stated.

The measurement of angles was done for solitary animals and for the groups (herd). When animals were seen in a group, the central animal was taken as a reference for angle measurement. I regarded individuals or groups as separate when they were $>30$ $\mathrm{m}$ apart from others. This distance was appropriate given the uneven distribution of animals on the plains. From the parameters above, the perpendicular distance $(\rho)$ was calculated as; $\rho=\chi_{\mathrm{i}} \sin \left(\theta_{i}\right)$ (Buckland et al., 2001). This perpendicular distance was used to model the detection function for the line transect. 


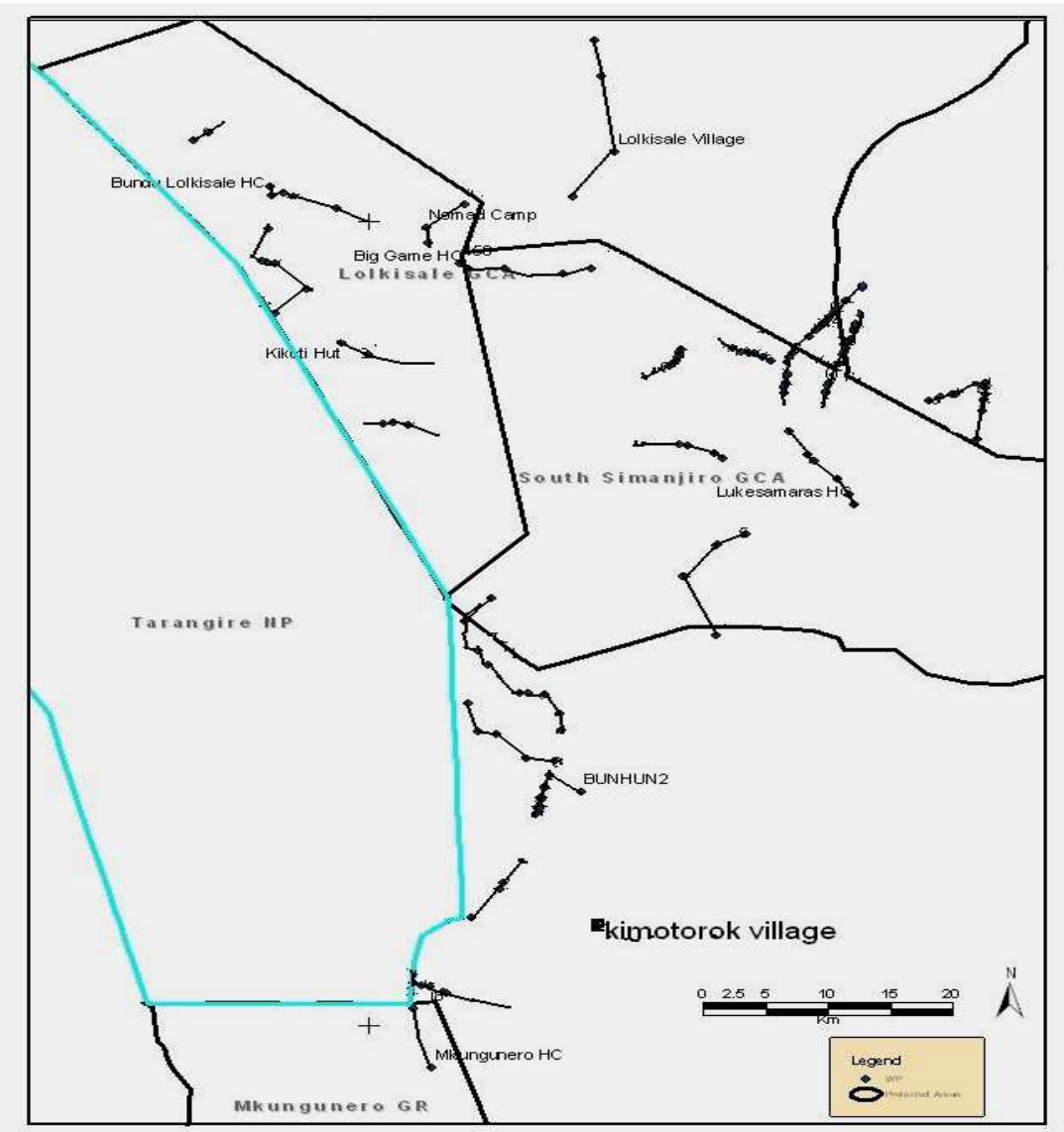

Figure 2. Location of Simanjiro study site showing distribution of transects (beaded lines) in the area.

\section{Data processing and analysis}

Prior to the analysis, data exploration and processing was done by testing out whether the critical assumptions for this method were met in the field. I plotted frequency histograms and scatter plots for all the species distance data to investigate the presence of evasive movements which violates the critical assumption 2 above (Buckland et al., 2001). In this phase, data heaping and outliers, factors that reduce the precision of model fit were checked. There were outliers only for impala. The impala's distance data were initially right-truncated to eliminate outliers and a suitable transect width was used for model fitting. Figure 3 shows different shapes of the detection function for each species' distance data. 

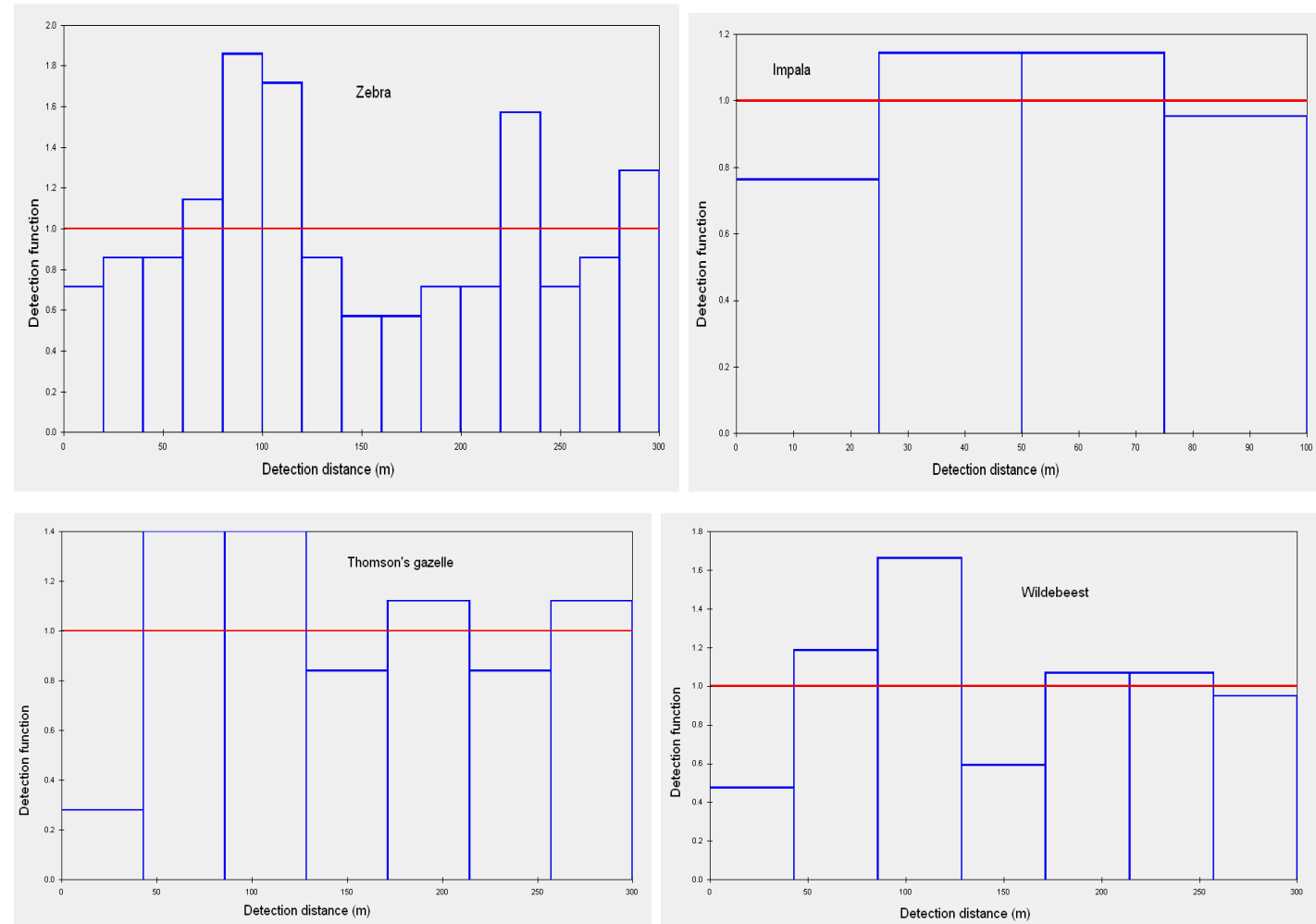

Figure 3. Observed frequency histogram of truncated distance data for zebra, impala, Thomson's gazelle and wildebeest.

Data were analysed using DISTANCE-vers. 5.0 Release 2 programme software (Thomas et al., 2008). Different detection models with series adjustment terms were used for analysis and compared to determine the best model suitable for each species data. Three detection functions (half-normal function with cosine/hermite polynomial adjustment, hazard-rate function with cosine/simple polynomial adjustment and a uniform function with cosine/simple polynomial adjustment) (Buckland et al., 2001) were initially used and compared their performances. Subsequent truncation of all the species data were carried out at specified distances based on the shapes of the detection functions (see Fig. 3). Right truncations were done at 300m, 100m, and $300 \mathrm{~m}$ and $300 \mathrm{~m}$ for the zebra, impala, Thomson's gazelle and wildebeest distance data respectively, to determine how the truncation influences the density estimates (Table 5.0). The model best fitting the data was selected by comparing the relative fit of these functions using the Akaike's Information Criterion (AIC) values (Buckland et al., 2001). The best density estimator was one with a minimum AIC. 
Table 5.0: Model performance and selection statistics for the zebra, impala, Thomson's gazelle and wildebeest distance data showing density estimates. A robust model is indicated with an asterisk (*). $\infty=$ Goodness of fit test.

\begin{tabular}{lllll}
\hline Model(key/adjustment) & AIC & Density & CV (\%) & p-valueo \\
\hline Zebra & & & & \\
30Om-truc+hazard rate/cosine & $1201.79^{*}$ & 19.69 & 37.6 & 0.14 \\
Hazard-rate/cosine & 1569.73 & 20.34 & 37.6 & 0.12 \\
Hazard-rate/simple polynomial & 1569.73 & 20.34 & 37.6 & 0.12 \\
Uniform/cosine & 1571.59 & 22.76 & 39.2 & 0.1 \\
Half-normal/cosine & 1572.85 & 23.88 & 42.3 & 0.07 \\
Half-normal/hermite & 1574.04 & 23.21 & 39.9 & 0.08 \\
Uniform+simple polynomial & 1574.81 & 25.24 & 38.1 & 0.06 \\
\hline Impala & & & & \\
\hline 100m-trunc+uniform/cosine & $193.42^{*}$ & 7.96 & 30.7 & 0.91 \\
300m trunc+hn/cosine & 379.68 & 8.07 & 30.7 & 0.65 \\
300m trunc+hn/hermite & 379.68 & 8.07 & 30.7 & 0.65 \\
300m trunc+hr/simple polyn. & 379.97 & 8.68 & 35.9 & 0.7 \\
300m trunc+hr/cosine & 379.97 & 8.68 & 35.9 & 0.7 \\
Hazard-rate/cosine & 403.48 & 8.59 & 32.7 & 0.11 \\
Half-normal/cosine & 406.29 & 8.62 & 29.5 & 0.08 \\
Half-normal/hermite & 407.78 & 6.97 & 27.7 & 0.16 \\
Uniform/cosine & 415.87 & 4.72 & 26.7 & 0 \\
Uniform/simple polynomial & 429.47 & 3.66 & 26.7 & 0 \\
\hline Thomson's gazelle & & & & \\
\hline 300m-trunc+uniform +cosine & $285.19^{*}$ & 2.45 & 35.1 & 0.35 \\
Uniform/cosine & 389.59 & 2.19 & 34.4 & 0.07 \\
Uniform/simple polynomial & 389.59 & 2.19 & 34.4 & 0.07 \\
Half-normal/cosine & 390.33 & 2.79 & 42.6 & 0.09 \\
Half-normal/hermite & 390.33 & 2.79 & 42.6 & 0.09 \\
Hazard-rate/cosine & 392.13 & 2.74 & 42.9 & 0.06 \\
Hazard-rate/simple polynomial & 392.13 & 2.74 & 24.9 & 0.06 \\
\hline Wildebeest & & & & \\
\hline 300m-trunc+uniform/cosine & $673.05^{*}$ & 9.12 & 51.4 & 25 \\
Hazard-rate/cosine & 797.17 & 8.92 & 54.6 & 01 \\
Hazard-rate/simple polynomial & 797.17 & 8.92 & 54.6 & 01 \\
Uniform/cosine & 798.08 & 9.17 & 55.6 & 02 \\
Uniform/simple polynomial & 798.08 & 9.17 & 55.6 & 02 \\
Half-normal/cosine & 798.08 & 9.17 & 55.6 & 02 \\
Half-normal/Hermite & 798.08 & 9.17 & 55.6 & 02 \\
\hline
\end{tabular}

For each species I estimated: (1) the encounter rate (number of herds per square kilometre with standard errors); (2) expected cluster size; (3) density of cluster; and (4) the overall density and abundance of the wildlife in the area. The model used for the final analysis was the uniform and hazard-rate key functions. These models showed improved density estimates for most of the species data (Table 5.0). 


\section{Results}

\section{Density of animals in the area}

The density estimates varies across the four species, with the zebra population showing the highest density $\left(19.69 / \mathrm{km}^{2}\right)$ than wildebeest $\left(9.12 / \mathrm{km}^{2}\right)$ and impala $\left(7.96 / \mathrm{km}^{2}\right)$. Thomson's gazelle appears to be the lowest dense animal in the area $(2.45$ $\mathrm{km}^{2}$ ). The different models tried for each of the species distance data, the fitted model functions and selection of the best and robust models for each species are shown in Table 5.0. According to Akaike's Information Criterion (AIC), two model key functions; a hazard rate and uniform keys appeared to fit the zebra data equally well. The hazard rate key model was used in final analysis as it is a relatively robust model over the uniform (Buckland et al. 2001). A uniform key model with cosine adjustments best fitted the impala and Thomson's gazelle distance data after truncation ( $p=0.91$ and $p=0.35$ respectively). On a truncated data, the uniform key function also was selected as the model that fitted the wildebeest grouped data $(p=0.25)$. The density and abundance of the four species are shown in Table 5.1.

Table 5.1: Density and abundance of wildlife species in the study area

\begin{tabular}{lllll}
\hline Species & Density (+S.E) & Abundance & \multicolumn{2}{l}{ 95\%Confidence interval } \\
\hline & & & LCL & UCL \\
\cline { 4 - 5 } Zebra & $19.69 \pm 7.39$ & 11223 & 5158 & 24218 \\
Wildebeest & $9.12 \pm 4.68$ & 5199 & 1842 & 14672 \\
Thomson's gazelle & $2.45 \pm 0.86$ & 1398 & 687 & 2843 \\
Impala & $7.96 \pm 2.45$ & 4534 & 2461 & 8354 \\
\hline
\end{tabular}

\section{Mean cluster size of the animals}

The mean herd size for each species observed in the area is shown in Table 5.2. The Thomson's gazelle was observed in smaller group size than the impala, wildebeest and zebra. The number of animals encountered per each square kilometre traversed, also differed across the four species. Zebra showed the highest encounter rate than impala and Thomson's gazelle. The encounter rate for wildebeest was twice that of the Thomson's gazelle (Table 5.2). There was no adjustment made for biases on the cluster size of the species distance data. 
Table 5.2: Estimated mean herd size, encounter rate (herds/ $\mathrm{km}^{2}$ ) and density of cluster with standard errors based on the final analysis after truncation.

\begin{tabular}{|c|c|c|c|c|}
\hline Species & $\begin{array}{l}\text { Number of } \\
\text { herds }\end{array}$ & $\begin{array}{l}\text { Mean } \\
\text { size }\end{array}$ & $\begin{array}{l}\text { Density of } \\
\text { cluster }\end{array}$ & $\begin{array}{l}\text { Encounter } \\
\text { rate }\end{array}$ \\
\hline Zebra & 105 & $13.28 \pm 1.11$ & $1.48 \pm 0.54$ & $0.89 \pm 0.05$ \\
\hline Wildebeest & 59 & $10.20 \pm 1.79$ & $0.89 \pm 0.43$ & $0.54 \pm 0.05$ \\
\hline Thomson's gazelle & 25 & $7.24 \pm 1.31$ & $0.34 \pm 0.10$ & $0.20 \pm 0.02$ \\
\hline Impala & 21 & $12.67 \pm 2.66$ & $0.63 \pm 0.14$ & $0.13 \pm 0.03$ \\
\hline
\end{tabular}

\section{Discussion}

\section{Zebra population}

The density of zebra in the area was higher than for other species. This is perhaps because of its widely spatial distribution resulting from temporally shifting of its range following the rain fall. This species exhibit a seasonal migration between the adjacent Tarangire National Park and nearby areas. Generally wild herbivores (zebra and wildebeest) use the Simanjiro plains as critical wet season dispersal range during the calving season (Lamprey, 1964). Zebra are virtually absent in this area during the mid to late dry seasons. The widespread distribution accounts for the high number of herds counted over each kilometre traversed.

The estimated density $\left(19.69 / \mathrm{km}^{2}\right)$ was influenced by the sample size and the effective strip width. Like, wildebeest and Thomson's, the effective strip width were relatively larger for zebra (300 mitres). Detection probability also contributed to the estimated high density zebra. The conspicuousness of the zebra skin and its relatively large body size probably increased its detectability even at far distances and thus minimised the effects of vegetation obscurity. The apparent effects of these parameters overall, are probably reflected in the wider effective strip-width and the large number of herds observed for this species.

The density of zebra reported in this study is remarkably different from other previous estimates done via aerial censuses in this area (Table 5.3). Lamprey (1964) used aerial strip count through southern Masailand, including part of Simanjiro and obtained a wet season dispersal density of one animal per $\mathrm{km}^{2}$. Two decades later, Kahurananga 
(1981) published a density of 10.96 zebra per $\mathrm{km}^{2}$ in the Simanjiro plains using aerial strip sampling. Since then there have been several counts attempted using aerial survey and results are summarised in Table 5.3.

Compared to the important grassland areas of East Africa, the estimated population of zebra is higher than $17.28 / \mathrm{km}^{2}$ and $8.0 / \mathrm{km}^{2}$ zebra for the Ngorongoro Crater and Serengeti-Mara ecosystem respectively (Estes et al., 2006; Osborne, 2000). It is however, lower compared to 30-45zebra/ $\mathrm{km}^{2}$ in Athi-Kapiti in Kenya (Prins et al., 2000). In the Masai-Mara and Koiyaki reserves, the zebra population was estimated at a density of 5.04 and $40.76 / \mathrm{km}^{2}$ during the wet(April) and dry(November) months respectively (Ogutu et al., 2006). This gives an overall abundance higher than of the present study. The vegetation structure in the Masai-Mara is about the same as that of the Simanjiro area and vast plains is characteristic in both cases. Despite these similarities, however, the Masai-Mara zebra population which transcends into the Serengeti is enormously large and alone could account for the differences between these studies.

Other studies of the savannah ungulates elsewhere in Africa provide comparable results. In Etosha national park Namibia, recent plains zebra population was estimated at 3700 animals (Gasaway et al., 1996). Unfortunately this population has continued to decline following persistent droughts in the region. Zebra is the lowest species in number(n=517) in Nyika N. Park, Malawi (Munthali \& Banda, 1992).

Table 5.3: Population estimates of wildlife in the Simanjiro plains from previous studies conducted using aerial census techniques in comparison with the present study. Data sources: * Kahurananga (1981), ** (TAWIRI, 1994 \& 2001) † present study.

\begin{tabular}{lllllll}
\hline Species & $\mathbf{1 9 7 0}^{\boldsymbol{*}}$ & $\mathbf{1 9 7 1}^{\boldsymbol{*}}$ & $\mathbf{1 9 7 2}^{\boldsymbol{*}}$ & $\mathbf{1 9 9 4}^{\star \star}$ & $\mathbf{2 0 0 1}^{\text {**}}$ & $\mathbf{2 0 0 8}^{\dagger}$ \\
\hline Zebra & $1787 \pm 274$ & $2747 \pm 250$ & $2698 \pm 553$ & $7787 \pm 2054$ & $6073 \pm 3896$ & $11223 \pm 4216$ \\
Wildebeest & $977 \pm 194$ & $2146 \pm 385$ & $2873 \pm 478$ & $6976 \pm 1863$ & $4189 \pm 2754$ & $5199 \pm 2670$ \\
T. gazelle & $194 \pm 59$ & $193 \pm 53$ & $162 \pm 61$ & --- & -- & $1398 \pm 491$ \\
Impala & $164 \pm 84$ & $105 \pm 43$ & $275 \pm 106$ & $1298 \pm 304$ & $1546 \pm 549$ & $4534 \pm 1393$ \\
\hline
\end{tabular}




\section{Wildebeest Population}

Wildebeest have the largest population second to zebra. Like other species the abundance of wildebeest is due to the variances in the herd size, encounter rate and effective strip width over which the species was counted. These parameters were larger for the wildebeest than for the impala and Thomson's gazelle but lower than for the zebra. Wildebeest exhibit similar distribution pattern and were observed in similar grazing range with zebra.

The estimated $5199\left(9.12 / \mathrm{km}^{2}\right)$ wildebeest in the present study is relatively similar to $5000\left(7.44 / \mathrm{km}^{2}\right)$ wildebeest previously published (Kahurananga, 1981) for the area. This density is also similar to 9.5 wildebeest per square kilometre in the Athi-Kapiti plains with similar ecological habitat in the eastern Masailand, Kenya (Talbot \& Talbot, 1963). The estimated density reported here is however, lower than the density $\left(52.0 / \mathrm{km}^{2}\right)$ of wildebeest in the Serengeti-Mara ecosystem (Osborne, 2000).The AthiKapiti wildebeest population is similar to that of Simanjiro because they all share the range with other herbivores species and with domestic livestock. In the Masai-Mara reserve Kenya, a survey conducted during the wet season as the present study did result in similar estimates (Ogutu et al., 2006). A total of 5109 wildebeest were estimated in the area. About 2600 wildebeest have been reported availably present in Etosha N. Park (Gasaway et al., 1996).

\section{Impala and Thomson's gazelle populations}

The population of these species were generally lower than of the zebra and wildebeest in the area. The low densities are probably attributable to their patchy distribution. As opposed to the wildebeest and zebra, both impala and Thomson's gazelle are resident species of the area. The Thomson's gazelle' range is highly localised only found in the central and southern plains of the study area. The population of these species might have been influenced by the human pressures in the area due to their habitats being degraded by farming. The encounter rate was almost equal for both impala and Thomson's gazelle. However, the Mean herd size for the Thomson's gazelle was almost half that of impala. This is probably due to the territorial behaviour of impala that warrants living in large closed groups. 
The density of these species was also influenced by effective strip width which was surprisingly larger for the Thomson's gazelle than for all other species investigated. This perhaps was due to the gazelle's coloration which increased the skin contrast against the background vegetation. The relatively smaller body size of the gazelle might have influenced its overall abundance negatively. However, because this species prefer and live mostly in open plains, the effect of its body size on its detectability was probably negligible.

The present densities of impala and Thomson's gazelle deduced are far higher than were reported in the previous studies. Kahunanga (1981) reported a density of $0.33 / \mathrm{km}^{2}$ and $0.28 / \mathrm{km}^{2}$ for the Thomson's gazelle and impala respectively. The differences between his estimates and the present ones may be due to the difference in the methods employed and may reflect improved conservation efforts in the area. In the Serengeti-Mara ecosystem, Thomson's gazelle was estimated at 17.6 animals per square kilometre (Osborne, 2000) higher than the present density in the Simanjiro plains. Generally the population of Thomson's gazelle and impala have been declining in major parts of their range due to increasing human pressures on their habitats.

\section{Caveats}

In this study I identify the following factors which were critical to reaching the precision of the density estimates sought.

1. Species grouping behaviour: For the zebra, Thomson's gazelle and wildebeest forming large and loose groups extending over a large distance is a characteristic in the Simanjiro plains and elsewhere. With this in mind it was difficult to establish the geometric centre of the groups and so was it hard to count them and measure the sighting distances with maximum accuracy. However, great care was taken to minimize bias that may have resulted from inaccurately recording of the necessary variables.

2. Species timidity: All the species investigated are behaviourally tame. However, long- time hunting in this area has probably caused them to learn to fear human beings in the area. Despite this, there is not evidence from distance data (Fig. 3) that animals moved away from transects prior to being detected and counted. 
3. Attaining large sample size for modelling the detection functions was difficult. Buckland et al. (2001) recommend a sample size of at least $60-80$ objects for modelling detection functions for a line transect. For rare species such as the Thomson's gazelle and naturally occurring grouped animals such as zebra and wildebeest that form loose agglomerations, fulfilling this need could be a daunting task. This could have caused some bias on the estimates reported here. Equally the same would be for species that exhibit seasonal migration between different range areas like the zebra and wildebeest.

During data collection for this study I increased sampling efforts to minimize the bias that would have resulted from this factor.

\section{Chapter summary}

Appropriate management of wildlife requires accurate population size and distribution data. I used Distance sampling method to estimate the density and abundance of four wild ungulate species; zebra, wildebeest, impala and Thomson's gazelle in the Simanjiro area, Tanzania. The uniform and hazard rate keys were used to model the detection functions for each species data. Results show that zebra is the most abundant animal $\left(19.69 / \mathrm{km}^{2}\right.$, SE 7.39$)$ followed by wildebeest $\left(9.12 / \mathrm{km}^{2}\right.$, SE 4.68$)$ and impala $\left(7.95 / \mathrm{km}^{2}, \mathrm{SE} 2.45\right)$. Thomson's gazelle is the least abundant in the area $\left(2.45 / \mathrm{km}^{2}\right.$, SE 0.86). The high number of zebra is attributable to its seasonal migratory behaviour from other areas into the Simanjiro plains during the study period. Resident species such as impala and Thomson's gazelle are few perhaps because of increased human activities in the area. Distance sampling method is recommended for use in subsequent regular monitoring of wildlife population by the authorities in order to track changes of wildlife population in the area.

\section{References}

Buckland, S.T.,Anderson, D.R.,Burham, K.P.,Laake, J.L.,Borchers, D.L., et al. (2001). Introduction to Distance Sampling:estimating abundance of biological populations. Oxford, UK: Oxford University Press.

Caro, T.M. (1999a). Densities of mammals in partially protected areas: The Katavi ecosystem of western Tanzania. The Journal of Applied Ecology, 36(2), 205217. 
Caro, T.M.,Pelkey, N.,Borner, M.,Severre, E.L.M.,Campbell, K.L.I., et al. (1998b). The impact of tourist hunting on large mammals in Tanzania: an initial assessment. African Journal of Ecology, 36(4), 321-346.

Dunbar, R.I.M. (1990). Environmental determinants of fecundity in klipspringer (Oreotragus oreotragus). African Journal of Ecology, 28(4), 307-313.

Estes, R.D.,Atwood, J.L., \& Estes, A.B. (2006). Downward trends in Ngorongoro Crater ungulate populations 1986-2005: Conservation concerns and the need for ecological research. Biological Conservation, 131(1), 106-120.

Gasaway, W.C.,Gasaway, K.T., \& Berry, H.H. (1996). Persistent low densities of plains ungulates in Etosha National Park, Namibia: testing the food-regulating hypothesis Canadian Journal of Zoology, 74(8), 1556-1572.

Kahurananga, J. (1981). Population estimates, densities and biomass of large herbivores in Simanjiro plains, northern Tanzania. African Journal of Ecology, 19(3), 225-238.

Komers, P.E. (1996). Obligate monogamy without paternal care in Kirk's dikdik. Animal Behaviour, 51(1), 131-140.

Lamprey, H.F. (1964). Estimation of the large mammal densities, biomass and energy exchange in the Tarangire game reserve and the Masai steppe in Tanganyika. African Journal of Ecology, 2(1), 1-46.

Munthali, S.M., \& Banda, H.M. (1992). Distribution and abundance of the common ungulates of Nyika National Park, Malawi. African Journal of Ecology, 30(3), 203-212.

Norton-Griffiths, M. (1978). Counting animals. Handbook no. 1. Techniques in African wildlife ecology. Nairobi: African Wildlife Foundation.

Ogutu, J.O.,Bhola, N.,Piepho, H.P., \& Reid, R. (2006). Efficiency of strip- and linetransect surveys of African savanna mammals. Journal of Zoology, 269(2), 149-160.

Osborne, L.P. (2000). Tropical ecosystems and ecological concepts. Cambridge University press. pp73-86.

Prins, H.H.T.,Grootenhuis, J.G., \& Dolan, T.T. (2000). Wildlife conservation by sustainable use. Springer. pp 146-166.

Talbot, L.M., \& Talbot, M.H. (1963). The wildebeest in western Masailand, East Africa. Wildlife Monographs(12), 3-88.

TAWIRI. (1994 \& 2001). Tanzania Wildlife Research Institute. Aerial wildlife census in Tarangire ecosystem. Arusha, Tanzania.

Thomas, L.,Laake, J.L.,Strindberg, S.,Marques, F.F.C.,Buckland, S.T., et al. (2008). Distance 5.0 release 2. Research unit for wildlife population assessment, University of St. Andrews, UK. (www.ruwpa.st-and.ac.uk/distancel). 


\section{MODELLING THE IMPACT OF HUNTING ON SPECIES POPULATION VIABILITY}

\section{Introduction}

Population viability analysis (PVA) is a tool for assessing the viability of populations under different demographic scenarios. It is usually conducted as simulation models used to make quantitative predictions about population size over time and the likelihood of extinction and examines effectiveness of alternative management options (Beissinger \& Westphal, 1998; Boyce, 1992; Marmontel et al., 1997). PVA models attempt to predict viability based on demographic data (such as censuses, mark-recapture studies, surveys and observation of reproduction and dispersal events, presence/absence data) and habitat data (Akcakaya \& Sjogren-Gulve, 2000; Brook et al., 2000; Haig et al., 1993).

The VORTEX computer simulation model (Lacy, 1993) is a Monte Carlo simulation of the effects of deterministic forces, as well as demographic, environmental and genetic stochastic events, on populations. The program allows input of actual life history parameters; reproductive rates, age class mortality rates, initial population size and carrying capacity as well as variations (plus standard deviations) in estimates of breeding, carrying capacity and mortality rates specified. External factors likely to impede population growth can also be modelled (e.g. disease and other natural phenomena) as catastrophes. The program also provides options for assessing human related impacts on populations such as harvesting, and can be specified across the various age class of the population being modelled.

Based on the input variables the program randomly changes the variables within a specified time limit and predicts the persistence or extinction risks within the time frame specified. After simulations, the output summary shows the probability of persistence, or if extinction, the number of populations that went extinct with the mean time to first extinction. Mean final population size at the end of simulation time with standard deviations, deterministic and stochastic population growth rates are also stated. The program also allows testing of different model parameters used to envisage most sensitive population parameters (sensitivity test). Here different management options available can be tested by allowing variations in demographic 
parameter inputs to evaluate those options (Lacy, 2000). Typical examples of the use of program-VORTEX to examine the impacts of human activities and natural phenomena are, Heinsohn et al. (2004) and Marmontel et al. (1997) who quantified the effects of hunting and human activities on dugongs (Dugong dugong) and the Florida manatee (Trichechus manatus latirostris) populations respectively, and predicted severe decline for both species. More widespread, however, is the use of population viability analysis for evaluating viability of small populations under threatened or fragmented habitats e.g. Haig et al. (1993)

My interest centres on species in Tanzania which are managed for exploitation. These species, which often provide both social and economic benefits to the human populations, need careful management strategies to ensure their long-term survival. The wildebeest and zebra are plentiful and are exploited through hunting both legally and illegally. Together with the impala and Thomson's gazelle, they face increasing pressures from offtake and habitat fragmentation by humans in the Tarangire region (Bolger et al., 2008; Newmark, 1996). The goal of this study was to investigate how the hunting will affect viability of wildlife populations in Tarangire. Therefore I used the program-VORTEX version 9.93 to examine the long-term impacts of hunting on the populations of impala, zebra, Thomson's gazelle and wildebeest outside Tarangire National Park, Tanzania.

I constructed models to examine:

1. The dynamics of the four species populations in Tarangire area without harvest, using demographic data and vital rates obtained from the area or if not available from other parts of eastern Africa.

2. The dynamics of the populations under the same demographic and vital rates but under the effects of legal and illegal hunting.

3. The most sensitive demographic parameters likely to influence the dynamics of the animal populations in the area.

\section{Methods}

\section{Sources of data}

The data used in the models include information gathered directly from the field as well as data retrieved from published literature. The initial population sizes are those 
derived from field estimations as described in Chapter 5 and from the estimation of the illegal and legal kill (Chapter 3\&2, (Table 6.0 below). For consistency, the legal hunting data used in the models was the average of three years of legal hunt not the five year data as collected from the field. This is because the illegal hunting data was estimated over a three year period only.

Table 6.0: Mean population sizes ( \pm standard errors) and species harvests for the Tarangire hunting area which were used in the models

\begin{tabular}{llll}
\hline Species & Illegal hunt & Legal hunt & Population size \\
\hline Zebra & 188 & 23 & $11223 \pm 4216$ \\
Wildebeest & 178 & 93 & $5199 \pm 2670$ \\
Thomson's gazelle & 197 & 57 & $1398 \pm 491$ \\
Impala & 226 & 75 & $4534 \pm 1393$ \\
\hline
\end{tabular}

In the absence of life history parameters for the Tarangire ungulate populations, I used data published for these species from other areas elsewhere in Africa as outlined in Chapter 4. I considered three reasons in selecting these vital rates: (i) similarities in plant phenology, (ii) local climatic conditions (rainfalls) and (iii) existing land-use types to suggest what these parameters would be for Tarangire. As shown in Chapter 4 , the life history parameters were collected from different populations across subSaharan Africa, but only a few areas closely match with Tarangire. The Serengeti ecosystem is similar to Tarangire both in plant phenology and local climates and vast plains are characteristic in all ecosystems (Lamprey, 1964). In terms of land-use, they are all protected as national parks and are in similar ecological regions receiving similar annual rainfalls of $855 \mathrm{~mm}$ and $721 \mathrm{~mm}$ for Serengeti and Tarangire respectively, (Grange \& Duncan, 2006). In the event where data were not available from northern Tanzania, nearby areas e.g. Athi-Kapiti plains in Kenya were used as they have somewhat similar vegetation structure (Talbot \& Talbot, 1963). Where these data were not available from these regions, I used any available data from other areas across the species' ranges.

Although the populations of my principal species in Tarangire and Serengeti National Parks might behave differently owing to the difference in predation potential, there is still no consensus on the impact of predators on the survival rates of zebra foals in 
Serengeti (Grange et al., 2004; Sinclair \& Norton-Griffiths, 1982). It appears also that the wildebeest and Thomson's gazelle populations in Serengeti are regulated by food supply rather than predators (Dublin et al., 1990; Mduma et al., 1999; Sinclair \& Norton-Griffiths, 1982). In Laikipia District (Kenya) where predators are not abundant rainfall influences the abundance of zebra (Georgiadis et al., 2003). Thus, I assumed that predation effects on populations in Serengeti and Tarangire national parks are not different. In the absence of data on mortality of wildebeest and zebra, I used the survival data for these species from Serengeti (Grange et al., 2004) to derive the age mortality rates for the Tarangire population (Heinsohn et al., 2004). Fecundity data for zebra and wildebeest were derived from the data given by Grange et al. (2004) and were used as surrogates for the Tarangire populations.

\section{Model Construction}

\section{Zebra PVA}

The life history parameters used for the zebra models are shown in Table 6.1.

Table 6.1: The ranges for the life history parameters of zebra as recorded in literature, and the values used in models in this study.

\begin{tabular}{lll}
\hline Parameter & Range/value & Values used \\
\hline Age at first reproduction for males (yr) & $3.0-4.0$ & 4 \\
Age at first reproduction for females (yr) & $3.0-4.0$ & 3 \\
Mating system & polygynous & polygynous \\
Number of young & 1 & 1 \\
Fecundity rate & $0.68-0.88$ & $0.7,0.8$ \\
Sex ratio of young (M/F) & 0.5 & 0.5 \\
Annual mortality (first year) & $0.19-0.3$ & 0.19 \\
Annual mortality (yearling) & 0.15 & 0.15 \\
Annual mortality (2 year old) & 0.02 & 0.02 \\
Annual mortality(3-5 year olds) & 0.05 & 0.05 \\
Annual mortality (6-10 year olds) & 0.13 & 0.13 \\
Annual mortality (adults) & 0.23 & 0.23 \\
Maximum age (yr) & $20-30$ & 22 \\
\hline
\end{tabular}

I constructed fourteen models simulating the population dynamics of zebra under different scenarios. The first model which allowed no harvest used all values as listed in Table 6.1 and assumed the female fecundity rate to be $80 \%$ (see Grange et al. 2004). In this and all other scenarios the population model assumed a stable distribution of age class, because no data are available on the age structure of the Tarangire ungulates. However, the available data indicate that fecundity for the 
Serengeti zebra is constant at age classes for females above five years old (Grange et $a l ., 2004)$, perhaps supporting the assumption of a stable age distribution as females start dropping their first foals when at age 3 years. I then reduced the female breeding to $70 \%$ (Model 2) and increased the mortality across all age classes by 10\% (Models $3,4)$ to test their effects on the population dynamics.

In subsequent models (Models 5-14), hunting was introduced into the model under varying female breeding and mortality rates as explained above. In these models, hunting was first set at $2 \%$ initial population size (i.e. 211 zebra from illegal and legal hunt) under $80 \%$ and $70 \%$ female breeding rates (Models 5, 6). I increased the mortality by $10 \%$ across all age classes and kept constant all other parameters as in previous models to test the effect of mortality (Models 7, 8). In models 9 and 10, I assumed the hunting level to be double the current rate and modelled the population under the two levels of female breeding and an additional 10\% mortality across all age classes as above. This simulated an increase in demand for zebra by both legal and illegal hunters in the area. I then modelled this population by factoring out the $10 \%$ mortality used in previous models (Models, 9, 10) to see how the population dynamics responds (Models 11, 12). In the last models (Models 13, 14), I examined the impacts of hunting by tripling the current hunting rate under the two levels of female breeding rates. In these models I used initial mortality rates with mortality increase as in previous models.

Variability in all life history parameters was set at 5\% of initial levels throughout all simulations. This is an arbitrary setting in order to introduce some variability into model simulations as most estimates of vital parameters (Chapter 4) do not provide variability estimates, and I have no idea of annual variability in vital parameters(see later discussion) in the Tarangire ecosystem. Further, there are no estimates of carrying capacity for any of the Tarangire species. For this species the carrying capacity was set at twice its initial population size. The population was thus modelled under density independence and the initial population size was set at the assessed field value of 11223 zebra. All the simulations excluded catastrophes, as there are no documented accounts of this occurring in Tarangire. Models also excluded inbreeding depression. I assumed the sex ratio at birth to be uniform (50\%), even though field 
data show adult sex ratio is biased towards females (Georgiadis et al., 2003). All simulations were run over 100 years and for 100 iterations each.

\section{Wildebeest PVA}

Table 6.2 shows the data used in all the wildebeest simulations.

Table 6.2: The ranges for the life history parameters of wildebeest as recorded in literature, and the values used in models in this study.

\begin{tabular}{lll}
\hline Parameter & Range/value & Values used \\
\hline Age at first reproduction for males (yr) & $3.0-4.0$ & 4 \\
Age at first reproduction for females (yr) & $2.0-3.0$ & 2 \\
Mating system & polygynous & polygynous \\
Number of young & 1 & 1 \\
Fecundity rate & $0.37-0.95$ & $0.7,0.8$ \\
Sex ratio of young (M/F) & 0.5 & 0.5 \\
Annual mortality (first year) & $0.16-0.25$ & 0.21 \\
Annual mortality (yearling) & 0.11 & 0.11 \\
Annual mortality (2 year old) & 0.13 & 0.13 \\
Annual mortality(3-5 year olds) & 0.11 & 0.11 \\
Annual mortality (6-10 year olds) & $0.21-0.28$ & 0.21 \\
Annual mortality (adults) & $0.22-0.37$ & 0.22 \\
Maximum age (yr) & 20 & 18 \\
\hline
\end{tabular}

All procedures for constructing the wildebeest model were the same as described for zebra except that for this species, the mortality values at age class one and two (yearlings) in Table 6.2 were increased by $20 \%$. This is because calf survival in Tarangire is approximately $20 \%$ lower than in the Serengeti wildebeest [(Lamprey (1962) in Talbot \& Talbot (1993)]. Similar to the model for zebra, additional mortality (10\%) was imposed across all age classes to explore its impact on the dynamics of wildebeest population. Hunting was first set at the assessed kill (i.e. 271 wildebeest from legal and illegal hunt, Models 5,6,7,8), then was increased by $50 \%$ to a total harvest of 407 wildebeest per year (Models 9, 10,11,12; Table 6.6). In the last two models, hunting was doubled (i.e. 542 wildebeest harvested per year) to test its impacts on the dynamics of the wildebeest population (Models, 13, 14). The initial population size used for this model was 5199 wildebeest as estimated from the field (Table 6.0).

\section{Thomson's gazelle PVA}

All data used in constructing the Thomson's gazelle model are shown in Table 6.3. 
Table 6.3: The ranges for the life history parameters of Thomson's gazelle as recorded in literature and the values used in models in this study.

\begin{tabular}{lll}
\hline Parameter & Range/value & Values used \\
\hline Age at first reproduction for males $(\mathrm{yr})$ & 2 & 2 \\
Age at first reproduction for females (yr) & 1 & 1 \\
Mating system & polygynous & polygynous \\
Number of young & 1 & 1 \\
Fecundity rate & 0.9 & $0.9,0.8$ \\
Sex ratio of young (M/F) & 0.5 & 0.5 \\
Annual mortality (first year) & $0.45-0.7$ & 0.45 \\
Annual mortality (yearling) & unknown & 0.15 \\
Annual mortality (3 year old) & unknown & 0.05 \\
Annual mortality(4-year olds) & unknown & 0.05 \\
Annual mortality (adults) & 0.22 & 0.22 \\
Maximum age (yr) & $10.5-12$ & 10 \\
\hline
\end{tabular}

The females of Thomson's gazelle are capable of breeding twice annually (Robinette \& Archer, 1971). In the absence of data on the fecundity for this species, I used a breeding rate of $90 \%$ as documented for dorcas gazelle (Gazella dorcas) (Yom-Tov et $a l ., 1995)$. The fecundity rate was also lowered to $80 \%$ for modelling purposes as described in the zebra model. I also used mortality values of 0.15 and 0.05 for yearling and age 3-4 year olds as observed in impala (Jarman \& Jarman, 1973) for a similar reason. An increased mortality (by 10\%) was imposed on initial mortality rates in Table 6.3 to examine its impacts on population dynamics.

Similar procedures to that described for zebra were used in constructing fourteen models for the Thomson's gazelle population. However, for this species, in models 914 inclusive, hunting was varied in a decreasing order of magnitude $-6 \%, 4 \%$ and $2 \%$ of the current population size instead of the $18 \%$ present hunting rate used in previous models $5-8$. This simulated hunting options likely to sustain this species in the area. The initial population size used was 1399 Thomson's gazelles (Table 6.0) and the carrying capacity was set at twice this number as in previous models.

\section{Impala PVA}

I constructed a density- independent model characterising the impala population dynamics using mortality and fecundity data shown in Table 6.4 below. 
Table 6.4: The ranges for the life history parameters of impala as recorded in literature and the values used in models in this study.

\begin{tabular}{lll}
\hline Parameter & Range/value & Values used \\
\hline Age at first reproduction for males (yr) & 2 & 5 \\
Age at first reproduction for females (yr) & 2 & 2 \\
Mating system & polygynous & polygynous \\
Number of young & 1 & 1 \\
Fecundity rate & $0.7-0.9$ & $0.7,0.8$ \\
Sex ratio of young (M/F) & 0.5 & 0.5 \\
Annual mortality (first year) & 0.4 & 0.4 \\
Annual mortality (2 year old) & $0.1-0.2$ & 0.1 \\
Annual mortality(3 year olds) & 0.05 & 0.05 \\
Annual mortality (4-5 year olds) & 0.03 & 0.05 \\
Annual mortality (6-8 year olds) & 0.05 & 0.25 \\
Annual mortality (adults) & $0.2-0.6$ & 0.25 \\
Maximum age (yr) & 13 & 11 \\
\hline
\end{tabular}

Fecundity is considered to be $70 \%$ and $90 \%$ in the first and later years respectively (Ginsberg \& Milner-Gulland, 1994). Males were assumed to start breeding at age five with only $30 \%$ of them participating in the breeding process (Jarman, 1979; Jarman \& Jarman, 1973). Thus my model assumed a maximum of $80 \%$ fecundity on average for all females and an alternative fecundity of $70 \%$ as in other species models. Yearling males (1-3 years olds) experience twice as high mortality than females (Dasmann \& Mossman, 1962; Jarman \& Jarman, 1973), so in the model input, the mortality of males at this age class was double that of females.

Using same procedure as described for zebra, I built fourteen models for impala and with a $10 \%$ increase in the mortality values across all age classes as in previous models. Hunting was examined using similar procedure as for Thomson's gazelle, where it was set at $6 \%, 4 \%$, and $2 \%$ of population size under models 8-14 inclusive. The initial population size for the impala model was 4534 animals.

\section{Results}

\section{Dynamics of animal populations under no harvest}

In the absence of harvest the simulated populations of all four species would survive for 100 years under models 1-4 inclusive (Tables $6.5 a-d$ ). The mean population sizes 
for all these species would stabilise under a no hunting regime and would remain just below the carrying capacity but above the initial population sizes for all the species.

Table 6.5a: Vortex simulation models for zebra population under different hunting scenarios showing the population growth $(r \pm S D)$, probability of extinction, mean extinction time and mean final population $( \pm S D)$. $F B=$ female breeding rate, $M=$ mortality

\begin{tabular}{llllll}
\hline & & $\begin{array}{l}\text { Mean } \\
\text { population } \\
\text { change }(\mathbf{r})\end{array}$ & $\begin{array}{l}\text { Probability } \\
\text { of } \\
\text { extinction }\end{array}$ & $\begin{array}{l}\text { Mean } \\
\text { extinction } \\
\text { time }(\mathbf{y r})\end{array}$ & $\begin{array}{l}\text { Mean final } \\
\text { population } \\
\text { size }\end{array}$ \\
\hline 1 & no hunt, 0.8 FB & $0.083 \pm 0.058$ & 0 & - & $22204 \pm 1093$ \\
2 & no hunt, 0.7 FB & $0.064 \pm 0.058$ & 0 & - & $22069 \pm 1157$ \\
3 & no hunt, 0.8 FB,+10\%M & $0.068 \pm 0.059$ & 0 & - & $22337 \pm 943$ \\
4 & no hunt, 0.7 FB,+10\%M & $0.048 \pm 0.059$ & 0 & - & $21729 \pm 1151$ \\
5 & hunt=211, 0.8 FB & $0.076 \pm 0.059$ & 0 & - & $22235 \pm 984$ \\
6 & hunt=211, 0.7 FB & $0.057 \pm 0.059$ & 0 & - & $22011 \pm 1244$ \\
7 & hunt=211, 0.8FB,+10\%M & $0.061 \pm 0.059$ & 0 & - & $22056 \pm 1121$ \\
8 & hunt=211,0.7 FB,+10\%M & $0.039 \pm 0.059$ & 0 & - & $21668 \pm 1240$ \\
9 & hunt=422, 0.8 FB & $0.068 \pm 0.059$ & 0 & - & $22061 \pm 1162$ \\
10 & hunt=422, 0.7 FB & $0.046 \pm 0.059$ & 0 & - & $21764 \pm 1156$ \\
11 & hunt=422,0.8 FB,+10\%M & $0.050 \pm 0.060$ & 0 & - & $21878 \pm 1329$ \\
12 & hunt=422,0.7 FB,+10\%M & $0.030 \pm 0.061$ & 0.01 & 72.0 & $20623 \pm 2715$ \\
13 & hunt=633,0.8 FB,+10\%M & $0.039 \pm 0.062$ & 0.01 & 36.0 & $21145 \pm 2513$ \\
14 & hunt=633,0.7 FB,+10\%M & $-0.008 \pm 0.095$ & 0.56 & 48.2 & $8764 \pm 10258$ \\
\hline
\end{tabular}

\section{Effects of hunting on population viability}

The introduction of harvest into the model systems resulted in considerable changes of mean population sizes of all the species (Models 5-14 inclusive, Tables 6.5a-d). Under initial harvest levels (Table 6.0), only the zebra population appears to be resilient and able to survive for 100 years with the mean population size hovering around the carrying capacity (Models 5,6,7,8; Table 6.5a). One of the simulated wildebeest populations went extinct (after 26 years) under the current hunting rate of approximately $5 \%$ of the initial population size (Model 8, Table 6.5b). By contrast, both Thomson's gazelle and impala populations struggled under their current hunting rates. The present level of harvest of Thomson's gazelle is $18 \%$, and under this regime $7 \%$ of its simulated populations went extinct. When a further $10 \%$ mortality was imposed, all simulated populations went extinct very rapidly (mean time to extinction 9.3 years; Models 5,6,7,8; Table 6.5c). Similarly, 6\% of the simulated impala populations went extinct under the present hunting regime of $6 \%$ of population size (model 5; Table 6.5d) and, alarmingly, a small reduction in female breeding rate 
(from $80 \%$ to $70 \%$ ) resulted in $76 \%$ of the simulated populations crashing. The addition of a further $10 \%$ mortality resulted in total population collapse (models 7,8 ; Table $6.5 d)$.

When the harvest levels were changed to simulate an increase or decrease in offtake for these species, their populations responded differently. Species for which hunting was simulated to increase above the present offtake (zebra and wildebeest), $1 \%$ and $4 \%$ of these populations went extinct (in mean times of 72.0 and 22.0 years respectively; models 9-12; Tables 6.5ab). For species whose levels of offtake were decreased showed mixed results. The survival of the Thomson's gazelle population was generally high and only $2 \%$ of its simulated populations went extinct when the hunting rate was reduced to $6 \%$ its initial population size (models 9-12 inclusive Table 6.5c). However, the populations of impala still declined to extinction when the hunting rates were reduced to $4.6 \%$ its initial current population size (models 9-12 inclusive; Table 6.5d).

The population of all species changed considerably with a further increase or decrease in the levels of offtake. The zebra population could no longer sustain any harvest if the offtake is tripled (to $5.6 \%$ its population size). Up to $56 \%$ of the simulated zebra populations went extinct under this level of hunting (models 13, 14; Table 6.5a). The probability of extinction was high $(P=0.85)$ for wildebeest when the hunting rate is doubled, about $10.4 \%$ its population size (models 13, 14; Table 6,5b). Decreasing of harvest to $2 \%$ would significantly improve the persistence of the Thomson's gazelle populations (models 13, 14; Table 6.5c). On the other hand, even if the offtake of impala was reduced to approximately $2 \%$ of its population size, $60 \%$ of the simulated populations went extinct. 
Table 6.5b: Vortex simulation models for wildebeest population under different hunting scenarios showing the population growth $(r \pm S D)$, probability of extinction, mean extinction time and mean final population $( \pm S D) . F B=$ female breeding rate, $M=$ mortality

\begin{tabular}{llllll}
\hline & & $\begin{array}{l}\text { Mean } \\
\text { population } \\
\text { change }(\mathbf{r})\end{array}$ & $\begin{array}{l}\text { Probability } \\
\text { of } \\
\text { extinction }\end{array}$ & $\begin{array}{l}\text { Mean } \\
\text { extinction } \\
\text { time }(\mathbf{y r})\end{array}$ & $\begin{array}{l}\text { Mean final } \\
\text { population } \\
\text { size }\end{array}$ \\
\hline 1 & no hunt, 0.8 FB & $0.095 \pm 0.062$ & 0 & - & $10301 \pm 536$ \\
2 & no hunt, 0.7 FB & $0.071 \pm 0.063$ & 0 & - & $10260 \pm 498$ \\
3 & no hunt, 0.8 FB,+10\%M & $0.076 \pm 0.064$ & 0 & - & $10212 \pm 554$ \\
4 & no hunt, 0.7 FB,+10\%M & $0.053 \pm 0.065$ & 0 & - & $10037 \pm 544$ \\
5 & hunt=271, 0.8 FB & $0.078 \pm 0.063$ & 0 & - & $10267 \pm 566$ \\
6 & hunt=271, 0.7 FB & $0.054 \pm 0.064$ & 0 & - & $10110 \pm 539$ \\
7 & hunt=271, 0.8FB,+10\%M & $0.059 \pm 0.065$ & 0 & - & $10137 \pm 570$ \\
8 & hunt=271,0.7 FB,+10\%M & $0.034 \pm 0.066$ & 0.01 & 26.0 & $9833 \pm 1193$ \\
9 & hunt=407, 0.8 FB & $0.070 \pm 0.065$ & 0 & - & $10247 \pm 531$ \\
10 & hunt=407, 0.7 FB & $0.043 \pm 0.068$ & 0.04 & 22.0 & $9551 \pm 2080$ \\
11 & hunt=407,0.8 FB,+10\%M & $0.050 \pm 0.067$ & 0 & - & $10049 \pm 687$ \\
12 & hunt $=407,0.7 \mathrm{FB},+10 \% \mathrm{M}$ & $0.015 \pm 0.082$ & 0.34 & 24.4 & $6456 \pm 4741$ \\
13 & hunt=542,0.8 FB,+10\%M & $0.034 \pm 0.081$ & 0.27 & 14.0 & $7143 \pm 4406$ \\
14 & hunt=542,0.7 FB,$+10 \% \mathrm{M}$ & $-0.029 \pm 0.122$ & 0.85 & 16.1 & $1369 \pm 3355$ \\
\hline
\end{tabular}

Table 6.5c: Vortex simulation models for Thomson's gazelle population under different hunting scenarios showing the population growth $(r \pm S D)$, probability of extinction, mean extinction time and mean final population $( \pm S D) . F B=$ female breeding rate, $M=$ mortality

\begin{tabular}{llllll}
\hline & & $\begin{array}{l}\text { Mean } \\
\text { population } \\
\text { change }(\mathbf{r})\end{array}$ & $\begin{array}{l}\text { Probability } \\
\text { of } \\
\text { extinction }\end{array}$ & $\begin{array}{l}\text { Mean } \\
\text { extinction } \\
\text { time (yr) }\end{array}$ & $\begin{array}{l}\text { Mean final } \\
\text { population } \\
\text { size }\end{array}$ \\
\hline 1 & no hunt, 0.9 FB & $0.156 \pm 0.073$ & 0 & - & $2803 \pm 138$ \\
2 & no hunt, 0.8 FB & $0.118 \pm 0.073$ & 0 & - & $2779 \pm 140$ \\
3 & no hunt, 0.9 FB,+10\%M & $0.115 \pm 0.075$ & 0 & - & $2757 \pm 149$ \\
4 & no hunt, 0.8 FB,+10\%M & $0.080 \pm 0.075$ & 0 & - & $2770 \pm 139$ \\
5 & hunt=254, 0.9 FB & $0.091 \pm 0.085$ & 0.07 & 12.1 & $2556 \pm 718$ \\
6 & hunt=254, 0.8 FB & $0.030 \pm 0.101$ & 0.68 & 15.0 & $864 \pm 1269$ \\
7 & hunt=254,0.9FB,+10\%M & $0.031 \pm 0.100$ & 0.64 & 13.9 & $965 \pm 1299$ \\
8 & hunt=254,0.8 FB,+10\%M & $-0.124 \pm 0.108$ & 1.00 & 9.3 & 0 \\
9 & hunt=84, 0.9 FB,+10\%M & $0.088 \pm 0.078$ & 0 & - & $2739 \pm 165$ \\
10 & hunt=84, 0.8 FB,+10\%M & $0.050 \pm 0.080$ & 0.02 & 49.5 & $2604 \pm 446$ \\
11 & hunt=56,0.9 FB,+10\%M & $0.099 \pm 0.077$ & 0 & - & $2775 \pm 152$ \\
12 & hunt=56,0.8 FB,+10\%M & $0.062 \pm 0.078$ & 0 & - & $2725 \pm 192$ \\
13 & hunt=28,0.9 FB,+10\%M & $0.106 \pm 0.077$ & 0 & - & $2770 \pm 129$ \\
14 & hunt=28,0.8 FB,+10\%M & $0.072 \pm 0.076$ & 0 & - & $2725 \pm 165$ \\
\hline
\end{tabular}


Table 6.5d: Vortex simulation models for the impala population under different hunting scenarios showing the population growth $(r \pm S D)$, probability of extinction, mean extinction time and mean final population $( \pm S D) . F B=$ female breeding rate, $M=$ mortality

\begin{tabular}{llllll}
\hline & & $\begin{array}{l}\text { Mean } \\
\text { population } \\
\text { change }(\mathbf{r})\end{array}$ & $\begin{array}{l}\text { Probability } \\
\text { of } \\
\text { extinction }\end{array}$ & $\begin{array}{l}\text { Mean } \\
\text { extinction } \\
\text { time (yr) }\end{array}$ & $\begin{array}{l}\text { Mean final } \\
\text { population } \\
\text { size }\end{array}$ \\
\hline 1 & nescription & $0.061 \pm 0.062$ & 0 & - & $8888 \pm 483$ \\
2 & no hunt, 0.8 FB & $0.036 \pm 0.061$ & 0 & - & $8635 \pm 491$ \\
3 & no hunt, 0.8 FB $+10 \% \mathrm{M}$ & $0.038 \pm 0.065$ & 0 & - & $8705 \pm 621$ \\
4 & no hunt, 0.7 FB,+10\%M & $0.013 \pm 0.064$ & 0 & - & $7770 \pm 1188$ \\
5 & hunt=301, 0.8 FB & $0.034 \pm 0.069$ & 0.06 & 34.8 & $8123 \pm 2158$ \\
6 & hunt=301, 0.7 FB & $-0.029 \pm 0.118$ & 0.76 & 35.9 & $1706 \pm 3206$ \\
7 & hunt=301,0.8FB,+10\%M & $-0.027 \pm 0.130$ & 0.75 & 36.5 & $1896 \pm 3400$ \\
8 & hunt=301,0.7 FB,+10\%M & $-0.108 \pm 0.154$ & 1.00 & 9.3 & 0 \\
9 & hunt=208, 0.8 FB,+10\%M & $0.038 \pm 0.065$ & 0.01 & 72.0 & $8699 \pm 1046$ \\
10 & hunt=208, 0.7 FB,+10\%M & $-0.038 \pm 0.167$ & 0.69 & 49.7 & $1947 \pm 3188$ \\
11 & hunt=208,0.8 FB, $+10 \% \mathrm{M}$ & $-0.011 \pm 0.133$ & 0.45 & 57.4 & $4169 \pm 4026$ \\
12 & hunt=208,0.7 FB,+10\%M & $-0.124 \pm 0.257$ & 1.00 & 32.0 & 0 \\
13 & hunt=104,0.8 FB,+10\%M & $0.1027 \pm 0.065$ & 0 & - & $8498 \pm 639$ \\
14 & hunt=104,0.7 FB, $+10 \% \mathrm{M}$ & $-0.033 \pm 0.137$ & 0.60 & 67.3 & $1911 \pm 2816$ \\
\hline
\end{tabular}

\section{Effects of varying mortality and fecundity rates on population dynamics}

The simulated populations of all species appeared to be strongly influenced by the fecundity and mortality rates used. In almost all species, the $10 \%$ variation in fecundity and mortality rates had little effect in the absence of harvest (models 1,2; Tables $6.5 a-d$ ) The mean final population sizes for these models decreased by 0.6 , 0.4, 0.9 and $2.8 \%$ from model one to model two for the zebra, wildebeest, Thomson's gazelle and impala respectively. Further, under the same hunting regime (Models $3 \& 4$, Tables $6.5 a-d$ ) an increase in mortality by $10 \%$ across all the age classes produced similar effects on the population sizes of these species except for the wildebeest.

\section{Sensitivity test}

I examined the effects of the variability associated with the vital parameters (fecundity and mortality rates). In the absence of field data indicating annual variability in fecundity and mortality estimates I had arbitrarily set this at 5\%, an admittedly small figure. I introduced a range of these values into the models for one species, wildebeest, as an example. These variations were set at 2.5, 5, 10, and $15 \%$ of the initial fecundity and mortality rates to test how the dynamics of this population responds. The population of wildebeest did not change under the lower (2.5 and 5\%) 
levels of these variations and the population survived to the last year of simulation (Table 6.6). The probability of survival decreased markedly at higher levels (i.e. 10$15 \%$ ). Increases of calf and yearling (2year olds) mortality rates by 10, 20 and $30 \%$ on initial levels did not affect the rate of survival of the wildebeest population (Table 6.6). However, the mortality affected growth rate (lambda) of this population which decreased with every increase of the mortality rates $(\lambda=1.072,1.064$ and 1.053 respectively) making this population more susceptible to extinction.

Table 6.6: Sensitivity of survival rates to increases of mortality (calf and yearling) and variation of mortality and fecundity rates. $C M=$ calf mortality

\begin{tabular}{|c|c|c|c|c|c|}
\hline Model & Description & $\begin{array}{l}\text { Mean } \\
\text { population } \\
\text { change }(r)\end{array}$ & $\begin{array}{l}\text { Probability } \\
\text { of extinction }\end{array}$ & $\begin{array}{l}\text { Mean } \\
\text { extinction } \\
\text { time (yr) }\end{array}$ & $\begin{array}{l}\text { Mean final } \\
\text { population } \\
\text { size }\end{array}$ \\
\hline 1 & $\begin{array}{l}\text { No hunt, } 2.5 \% \\
\text { variation } \\
\text { No hunt, } 5 \%\end{array}$ & $0.096 \pm 0.031$ & 0 & - & $10367 \pm 275$ \\
\hline 2 & $\begin{array}{l}\text { variation } \\
\text { No hunt, } 10 \%\end{array}$ & $0.094 \pm 0.063$ & 0 & - & $10271 \pm 488$ \\
\hline 3 & $\begin{array}{l}\text { variation } \\
\text { No hunt, } 15 \%\end{array}$ & $0.091 \pm 0.129$ & 0 & & $9656 \pm 1362$ \\
\hline 4 & $\begin{array}{l}\text { variation } \\
\text { hunt }=271,2.5 \%\end{array}$ & $0.074 \pm 0.211$ & 0.01 & 57 & $8115 \pm 2619$ \\
\hline 5 & $\begin{array}{l}\text { variation } \\
\text { hunt }=271,5 \%\end{array}$ & $0.080 \pm 0.033$ & 0 & - & $10389 \pm 241$ \\
\hline 6 & $\begin{array}{l}\text { variation } \\
\text { hunt }=271,10 \%\end{array}$ & $0.079 \pm 0.064$ & 0 & - & $10343 \pm 551$ \\
\hline 7 & $\begin{array}{l}\text { variation } \\
\text { hunt }=271,15 \%\end{array}$ & $0.078 \pm 0.069$ & 0.03 & 15 & $9271 \pm 2240$ \\
\hline 8 & $\begin{array}{l}\text { variation } \\
\text { hunt }=90,2.5 \%\end{array}$ & $0.040 \pm 0.233$ & 0.4 & 52 & $4407 \pm 4267$ \\
\hline 9 & $\begin{array}{l}\text { variation } \\
\text { hunt }=90,5 \%\end{array}$ & $0.088 \pm 0.032$ & 0 & - & $10419 \pm 269$ \\
\hline 10 & $\begin{array}{l}\text { variation } \\
\text { hunt }=90,10 \%\end{array}$ & $0.087 \pm 0.062$ & 0 & - & $10237 \pm 473$ \\
\hline 11 & $\begin{array}{l}\text { variation } \\
\text { hunt }=90,15 \%\end{array}$ & $0.078 \pm 0.133$ & 0 & - & $9250 \pm 1272$ \\
\hline 12 & variation & $0.057 \pm 0.215$ & 0.07 & 72 & $7411 \pm 3537$ \\
\hline 13 & No hunt, $+10 \% \mathrm{CM}$ & $0.086 \pm 0.063$ & 0 & - & $10324 \pm 327$ \\
\hline 14 & No hunt, $+20 \% \mathrm{CM}$ & $0.077 \pm 0.064$ & 0 & - & $10307 \pm 319$ \\
\hline 15 & No hunt, $+30 \% \mathrm{CM}$ & $0.069 \pm 0.064$ & 0 & - & $10234 \pm 336$ \\
\hline
\end{tabular}

\section{Discussion}

The simulation models suggest that the impala and Thomson's gazelle are susceptible to present levels of harvest and are at great risks of severe decline. Even for wildebeest and zebra, whose simulated populations appeared to withstand the present levels of hunting, severe population declines would occur if harvesting is increased 
above their current levels. If these models are, or near correct, then the impala and Thomson's gazelle populations will decline towards extinction within a relatively short time (15 years). These results confirm the suggestions by Caro et al. (1998b) that hunting is causing the decline of these species in the Tarangire hunting zone.

Illegal and legal hunting has been implicated as causing declines of mammal populations in several Tanzanian National Parks and surrounding areas. Poaching severely reduced the population of buffalo (Syncerus caffer) in northern Serengeti (Sinclair, 1995) and trophy hunting has been suggested to be the cause of declining wildlife populations across large parts of Tanzania (Caro et al., 1998b). Although legal hunting is (meant to be) highly selective for adult males, in the presence of high offtake by citizens and illegal hunters, this strategy may be flawed. Without supervision and with opportunistic poaching, both males and female would be hunted. This would increase total offtake and directly reduce the number of animals in the area.

Selective hunting as a way of sustaining the breeding potential of exploitable populations is still debated. Selective hunting of adult males has caused retarded female conception and reproductive collapse in Saiga antelopes (Milner-Gulland et al., 2003; Milner-Gulland et al., 2001). Disproportionate hunting of prime males of sable antelope in northern Zimbabwe was also suggested to have been responsible for the reduced survivorship of young due to extended parturition period and for causing a population decline (Fergusson, 1990 in Ginsberg \& Milner-Gulland, 1994). In impala population, Ginsberg \& Milner-Gulland (1994) also reported unsustainable hunting resulted due to selecting adult males.

Furthermore, the term 'adult male' seems ambiguous especially during the actual hunting process in the field. Due to the difficulty of ageing animals in the field, active prime males would almost certainly be killed. This could result in retarded female conception and young survival and it is not clear whether, and at what level, this may apply in the Tarangire hunted populations. But hunting models strongly suggest that the manipulation of sex ratio occurring in hunted populations could lead to reduced 
fecundity and high probability of population collapse (Ginsberg \& Milner-Gulland, 1994; Gruver et al., 1984).

\section{Model Caveats}

This is the first and initial attempt to characterise the dynamics of the Tarangire populations under best and worst cases possible.

In the absence of life history parameters for the Tarangire populations, it seems inappropriate to attempt any studies modelling population dynamics. While this may be true, however, Boyce (1992) made a point that one should do the best with whatever limited information available to serve threatened species.

Therefore the analyses presented here are underpinned by several assumptions:

(i) Life history parameters of the four study populations from other areas are the same, or similar, to those at Tarangire. As this assumption may at times be flawed due to extreme variations in local conditions between regions, however I chose to use parameters documented from populations that are situated close to Tarangire National Park to minimise this effect. Further, in the simulation models I allowed variation of most sensitive life history parameters, fecundity and mortality rates under what I considered to be 'best' and 'worst' case scenarios. For all species models, fecundity was first assumed maximum by using highest fecundity rate observed in other areas, and alternative models were built assuming a lowered breeding rate. Mortality was also modelled by using alternative mortality rates and I assume the Tarangire population will be somewhere within these range of mortality and fecundity used in the simulations. In the event that even optimistic models show a high probability of extinction, it is certain that hunting is having negative impacts on the populations (Heinsohn et al., 2004). The simulated models of impala and Thomson's gazelle indicate that these species are not faring well under even the most optimistic scenarios (Tables, 6.5c,d), suggesting strongly that the current harvest of these species is unsustainable.

(ii) The population simulations assumed the absence of catastrophes in all species populations in the Tarangire area. Catastrophes have additive 
effects on the dynamics of populations through retarding population growth and thus reducing their long-term viability (Young, 1994). There have been no documented accounts of catastrophes in any of my study populations although impala at nearby Lake Manyara National Park have suffered an anthrax outbreak in the 1980s that reduced their population severely (Prins \& Weyerhaeuser, 1987). However, as Young (1994) reviews, starvation (drought) has been a major constraint of herbivores populations in many tropical arid regions, and the Tarangire populations may not be exceptional. There are occasional extreme drought conditions occurring in the study area (pers.observation, 2005), but their impacts on the wildlife populations are known.

(iii) The models assumed a uniform variability associated with key parameter inputs of fecundity, mortality and carrying capacity. I set this variability at a low $5 \%$, probably too low considering the impact of human populations on the wildlife habitats in the area. There have been disruptions of wildlife migratory corridors connecting Tarangire Park and adjacent dispersal areas due to expansion of agricultural fields that have increased to six times that used in 1940s (Gamassa, 1995; Mwalyosi, 1991). As a consequence, between 1988 and 2001, the population of wildebeest and other species declined by $88 \%$ in the Tarangire ecosystem (TAWIRI, 2001). While the impacts of habitat loss on fecundity and mortality rates are still unclear, when I increased the variability of the vital parameters in the simulated populations the decline in wildebeest populations was substantial (Table 6.6).

(iv) The models do not include density dependence nor do they incorporate genetics. Environmental variation and density-dependence are important limiting factors on the juvenile survival and age at first reproduction of large mammal herbivores (Gaillard et al., 2000). Weather and density tend to first affect the juvenile survival and fecundity of young females and often influences population growth rates (Gaillard et al., 2000). At high density, adult survival also influences growth of a population (Albon et al., 2000). Thus in hunted populations, there are risks of compromising adult 
survival hence potentially limiting population growth rate of the harvested population.

Although this study borrows life history data (vital rates) from other populations, it provides useful information that highlights the current and future likely condition of the Tarangire wildlife. The underlying assumptions of these models cannot be evaluated unless deliberate efforts are made to document these parameters at Tarangire. The results therefore, are a warning to the managers of reserves that these species may not be safe from extinction under present levels of hunting.

\section{Implications for conservation and sustainable harvest in Tarangire}

The results of this study highlight the need for immediate policy to regulate hunting of impala and Thomson's gazelle populations in the Tarangire area. According to the simulated populations, these species appear unable to sustain present levels of harvest without experiencing severe declines leading to population collapse. As poachers and legal hunters continue to remove these animals, they make these populations ever more susceptible to extinction. Additionally, important wildlife habitat continues to be lost through conversion to agricultural lands (Bolger et al., 2008; Gamassa, 1995; Mwalyosi, 1992). The fate of these animal populations is equivocal!

The current harvest levels of 5\% and 18\% for impala and Thomson's gazelle are unsustainable and need to be reduced to avoid any risks of losing these species. According to the simulation models these species would persist to 100 years and beyond when the harvest is stopped or levels kept below $2 \%$ and at $4 \%$ of population sizes of impala and Thomson's gazelle respectively. Reduction of total harvest of wildebeest and zebra is also recommended. However, reducing the harvest levels alone will not serve these species for longer times. Instead, efforts should be made to greatly reduce habitat loss for these species. Therefore policies pertinent to land ownership and agriculture should be reviewed to include measures targeted at retaining wildlife habitats. It is only when these issues are effectively addressed that conservation prospects of the wildlife can be realised.

Poaching appears to remove large numbers of animals, many times more than the legal harvest. While it will be difficult to completely remove poaching given that 
poachers do not identify themselves it is essential to combat this problem. Even complete cessation of legal hunting will not lower the offtake to the level that these populations might be able to sustain. However, a long-term education plan, enforcement of protection rules, and community involvement in conservation of wildlife should prove fruitful especially in those areas that are not formerly protected as national parks. Policy shift in favour of protecting the wildlife on such lands needs more emphasis.

Accurate population data for harvested species is required to detect changes of these populations over time. As the available data (mostly from aerial surveys) are for few areas and mostly national parks and few reserves more work is needed to increase documentation of the population size of these species. These data need to be complemented by ground surveys in all wildlife-rich areas. One way to do this is for wildlife managers to actively be involved in counting of these animals for their reserves.

\section{Practical application of VORTEX for managing Tarangire wildlife populations}

VORTEX is recommended as a tool for managing harvested wildlife populations in the Tarangire region in Tanzania. Although important data for developing VORTEX models for these populations are not available, this would not prevent wildlife managers from using this tool for managing these animals. Initial models could be built using surrogate data, as I have done. By constructing a VORTEX model for each species, it would help managers and researchers identify immediate information needs and prioritise research. Data collection would continue that are aimed at informing a VORTEX model for a particular species, e.g. impala. Such models would be improved incrementally as more data become available.

This study has identified that the simulated populations of these species are most sensitive to fecundity and natural mortality rates across all age class and to the annual variability associated with them. The lessons that are borne out from these models are that, wildlife managers and researchers who are responsible for these animals in Tarangire and elsewhere in Tanzania should be aware of the importance of these parameters and the urgency of measuring these vital rates in the field. 
To ensure effective implementation of the management of these animals through VORTEX- models, it is important that this goal be defined and incorporated in the general management plan (GMP) documents of each national park and game reserve. Thereafter routine collection of data would be needed to inform these models. For any species model developed for such purpose would require the following for effective management of these species.

1. Collating data on species vital parameters (fecundity and mortality rates) most likely to influence the dynamics of these populations. However, because clear understanding and documentation of these variables would require qualified biologists and longer time of field research, it is unlikely that this work could be done by the wildlife managers and park wardens alone. Alternative ways of approaching this would be to give reserve managers and national park wardens and ecologists little training on how to conduct proper field population census surveys. Ground surveys are most recommended and are easier to do especially for species that are difficult to count from the air. This work should be defined on a to-do list of wardens' job and should be done twice or at least once annually. This would prove effective and ensure longterm documentation of population size of the animals in these reserves. The presence of a long-term population data would help calculate some of the parameters required to inform VORTEX management models.

2. Accurately recording of number of animals killed by hunting. There appears to be ineffective documentation of the animals killed in most reserves. Close supervision of resident hunting is needed as this has been identified to be lacking proper documentation and the accurate number of animals killed under this category is not known. Equally important is illegal offtake from these reserves. Most management decisions ignore this either due to difficulties in quantifying it or because they assume its impact is less important. As this study indicates, illegal hunting is equally important as legal offtake in terms of its impacts on the population size of these species. Reliable methods should be employed to quantify the size of illegal offtake in order to inform VORTEX-management models.

3. Accurately recoding age and condition of the animals killed by legal hunters. Hunting of 'adult males' is a lose term and potentially risks killing of prime males. Studies (Ginsberg \& Milner-Gulland, 1994; Gruver et al., 1984; 
Milner-Gulland et al., 2003) have shown how the hunting of prime males increases susceptibility of a population to reproductive collapse. Therefore to ensure accurate data of the animals killed, supervisors and observers involved in the hunting process need training on how to accurately age the animals and collect these data in the field. This would help inform the management models with great accuracy.

4. Recording and documenting the number of animals dying under natural death such as diseases and starvation would also improve the data needed for a particular species management model.

To efficiently apply VORTEX-model as a tool for managing these species needs commitment and financial investment for the government as well as reserve managers and park wardens. One way would be to start with the Tarangire population as a pilot project study and later carry on with other areas as this tool become more familiar to many personnel. It is only when there is clear understanding of the variables most driving the dynamics of these populations that reliable and effective management decisions can be made that will ensure long-term persistence of the exploited wildlife population in Tanzania.

\section{Chapter summary}

I examined the long-term impacts of continued harvest of four ungulate species available in the Tarangire hunting area using surrogate vital life history data, mostly from nearby Serengeti National Park, Tanzania. I used VORTEX to construct models to characterise the dynamics of these populations under a range of hunting regimes. The model results suggest that the present levels of hunting of impala and Thomson's gazelle are unsustainable. While hunting of these species is kept at $2 \%$ and $6 \%$ of current population sizes respectively, the impala and Thomson's gazelle populations will slowly decline towards extinction. The populations of wildebeest and zebra show greater resilience under most model scenarios. Zebra persistence remains high under the current hunting rate of approximately $2 \%$ its population size. However, its population will start declining if the offtake exceeds this rate. Under the present $5 \%$ harvest regime, wildebeest will also decline if calf mortality exceeds $20 \%$ the present assumed rates. Lower harvest rate for all species is advisable and VORTEX is recommended for use as a tool for managing the Tarangire exploited populations. 


\section{References}

Akcakaya, H.R., \& Sjogren-Gulve, P. (2000). Population viability analyses in conservation planning: an overview. Ecological Bulletins, 48, 9-21.

Albon, S.D.,Coulson, T.N.,Brown, D.,Guinness, F.E.,Pemberton, J.M., et al. (2000). Temporal changes in key factors and key age groups influencing the population dynamics of female red deer. Journal of Animal Ecology, 69(6), 1099-1110.

Beissinger, S.R., \& Westphal, M.I. (1998). On the use of demographic models of population viability in endangered species management. The Journal of Wildlife Management, 62(3), 821-841.

Bolger, D.T.,Newmark, W.D.,Morrison, T.A., \& Doak, D.F. (2008). The need for integrative approaches to understand and conserve migratory ungulates. Ecology Letters, 11(1), 63-77.

Boyce, M.S. (1992). Population viability analysis. Annual Review of Ecology and Systematics, 23(1), 481-497.

Brook, B.W.,O'Grady, J.J.,Chapman, A.P.,Burgman, M.A.,Akcakaya, H.R., et al. (2000). Predictive accuracy of population viability analysis in conservation biology. Nature, 404, 385-387.

Caro, T.M.,Pelkey, N.,Borner, M.,Severre, E.L.M.,Campbell, K.L.I., et al. (1998b). The impact of tourist hunting on large mammals in Tanzania: an initial assessment. African Journal of Ecology, 36(4), 321-346.

Dasmann, R.F., \& Mossman, A.S. (1962). Population studies of impala in southern Rhodesia. Journal of Mammalogy, 43(3), 375-395.

Dublin, H.T.,Sinclair, A.R.E.,Boutin, S.,Anderson, E.,Jago, M., et al. (1990). Does competition regulate ungulate populations? Further evidence from Serengeti, Tanzania. Oecologia, 82(2), 283-288.

Gaillard, J.-M.,Festa-Bianchet, M.,Yoccoz, N.G.,Loison, A., \& Toigo, C. (2000). Temporal variation in fitness components and population dynamics of large herbivores. Annual Review of Ecology and Systematics, 31(1), 367-393.

Gamassa, D.G.M. (1995). Blockade of wildlife migration corridors by agricultural development in northern Tanzania. In: Integrating people and wildlife for a sustainable future (eds. Bissonette, J.A. \& Krausman, P.R.). The Wildlife Society, Bethesda, pp. 609-613.

Georgiadis, N.,Hack, M., \& Turpin, K. (2003). The influence of rainfall on zebra population dynamics: implications for management. The Journal of Applied Ecology, 40(1), 125-136.

Ginsberg, J.R., \& Milner-Gulland, E.J. (1994). Sex-biased harvesting and population dynamics in ungulates: implications for conservation and sustainable use. Conservation Biology, 8(1), 157-166.

Grange, S., \& Duncan, P. (2006). Bottom-up and top-down processes in African ungulate communities: resources and predation acting on the relative abundance of zebra and grazing bovids. Ecography, 29(6), 899-907.

Grange, S.,Duncan, P.,Gaillard, J.-M.,Sinclair, A.R.E.,Gogan, P.J.P., et al. (2004). What limits the Serengeti zebra population? Oecologia, 140(3), 523-532.

Gruver, B.J.,Guynn, D.C., Jr., \& Jacobson, H.A. (1984). Simulated effects of harvest strategy on reproduction in white-tailed deer. The Journal of Wildlife Management, 48(2), 535-541. 
Haig, S.M.,Belthoff, J.R., \& Allen, D.H. (1993). Population viability analysis for a small population of red-cockaded woodpeckers and an evaluation of enhancement strategies. Conservation Biology, 7(2), 289-301.

Heinsohn, R.,Lacy, R.C.,Lindenmayer, D.B.,Marsh, H.,Kwan, D., et al. (2004). Unsustainable harvest of dugongs in Torres Strait and Cape York (Australia) waters: two case studies using population viability analysis. Animal Conservation, 7(4), 417-425.

Jarman, M.V. (1979). Impala social behaviour : territory, hierarchy, mating, and the use of space. Berlin; Hamburg: Parey.

Jarman, P.J., \& Jarman, M.V. (1973). Social behaviour, population structure and reproductive potential in impala. African Journal of Ecology, 11(3-4), 329338.

Lacy, R.C. (1993). VORTEX: a computer simulation model for population viability analysis. Wildlife Research, 20(1), 45-65.

Lacy, R.C. (2000). Structure of the VORTEX simulation model for population viability analysis. Ecological Bulletins, 48, 191-203.

Lamprey, H.F. (1964). Estimation of the large mammal densities, biomass and energy exchange in the Tarangire game reserve and the Masai steppe in Tanganyika. African Journal of Ecology, 2(1), 1-46.

Marmontel, M.,Humphrey, S.R., \& O'Shea, T.J. (1997). Population viability analysis of the Florida manatee (Trichechus manatus latirostris), 1976 -1991. Conservation Biology, 11(2), 467-481.

Mduma, S.A.R.,Sinclair, A.R.E., \& Hilborn, R. (1999). Food regulates the Serengeti wildebeest: a 40-year record. Journal of Animal Ecology, 68(6), 1101-1122.

Milner-Gulland, E.J.,Bukreeva, O.M.,Coulson, T.,Lushchekina, A.A.,Kholodova, M.V., et al. (2003). Conservation: reproductive collapse in saiga antelope harems. Nature, 422, 135-135.

Milner-Gulland, E.J.,Kholodova, M.,Bekenov, A.B.,Bukreeva, O.,Grachev, Y., et al. (2001). Dramatic declines in saiga antelope populations. Oryx, 35(4), 340-345.

Mwalyosi, R.B.B. (1991). Population growth, carrying capacity and sustainable development in south-west Masailand. Journal of Environmental Management, 33(2), 175-187.

Mwalyosi, R.B.B. (1992). Land-use changes and resource degradation in south-west Masailand, Tanzania. Environmental Conservation, 19(2), 145-152.

Newmark, W.D. (1996). Insularization of Tanzanian parks and the local extinction of large mammals. Conservation Biology, 10(6), 1549-1556.

Prins, H.H.T., \& Weyerhaeuser, F.J. (1987). Epidemics in populations of wild ruminants: anthrax and impala, rinderpest and buffalo in Lake Manyara National Park, Tanzania. Oikos, 49(1), 28-38.

Robinette, W.L., \& Archer, A.L. (1971). Notes on ageing criteria and reproduction of Thomson's gazelle. African Journal of Ecology, 9(1), 83-98.

Sinclair, A.R.E. (1995). Population limitation of resident herbivores. In: Serengeti II: dynamics, management, and conservation of an ecosystem (Eds A.R.E. Sinclaire and P. Arcese).University of Chicago Press, Chicago.

Sinclair, A.R.E., \& Norton-Griffiths, M. (1982). Does competition or facilitation regulate migrant ungulate populations in the Serengeti? A test of hypotheses. Oecologia, 53(3), 364-369.

Talbot, L.M., \& Talbot, M.H. (1963). The wildebeest in western Masailand, East Africa. Wildlife Monographs(12), 3-88. 
TAWIRI. (2001). Tanzania Wildlife Research Institute. Aerial wildlife census in Tarangire ecosystem. Arusha, Tanzania.

Yom-Tov, Y.,Mendelssohn, H., \& Groves, C.P. (1995). Gazella dorcas Mammalian species, 491, 1 - 6 .

Young, T.P. (1994). Natural die-offs of large mammals: implications for conservation. Conservation Biology, 8(2), 410-418. 